\title{
The oleaginous astaxanthin-producing alga Chromochloris zofingiensis: potential from production to an emerging model for studying lipid metabolism and carotenogenesis
}

\author{
Yu Zhang, Ying Ye, Fan Bai and Jin Liu* (D)
}

\begin{abstract}
The algal lipids-based biodiesel, albeit having advantages over plant oils, still remains high in the production cost. Co-production of value-added products with lipids has the potential to add benefits and is thus believed to be a promising strategy to improve the production economics of algal biodiesel. Chromochloris zofingiensis, a unicellular green alga, has been considered as a promising feedstock for biodiesel production because of its robust growth and ability of accumulating high levels of triacylglycerol under multiple trophic conditions. This alga is also able to synthesize high-value keto-carotenoids and has been cited as a candidate producer of astaxanthin, the strongest antioxidant found in nature. The concurrent accumulation of triacylglycerol and astaxanthin enables C. zofingiensis an ideal cell factory for integrated production of the two compounds and has potential to improve algae-based production economics. Furthermore, with the advent of chromosome-level whole genome sequence and genetic tools, C. zofingiensis becomes an emerging model for studying lipid metabolism and carotenogenesis. In this review, we summarize recent progress on the production of triacylglycerol and astaxanthin by C. zofingiensis. We also update our understanding in the distinctive molecular mechanisms underlying lipid metabolism and carotenogenesis, with an emphasis on triacylglycerol and astaxanthin biosynthesis and crosstalk between the two pathways. Furthermore, strategies for trait improvements are discussed regarding triacylglycerol and astaxanthin synthesis in C. zofingiensis.
\end{abstract}

Keywords: Abiotic stress, Astaxanthin, Chromochloris zofingiensis, Metabolic engineering, Omics, Triacylglycerol

\section{Background}

Up to date, the unsustainable fossil fuels have still served as the main global energy sources and their growing consumption leads to increasing emission of carbon dioxide into the atmosphere and thus severe environmental problems that threaten our ecosystem [1]. The utilization of alternative energy sources that are renewable and

${ }^{*}$ Correspondence: gjinliu@pku.edu.cn

Laboratory for Algae Biotechnology and Innovation, College

of Engineering, Peking University, Beijing 100871, China carbon neutral represents a feasible way toward reducing carbon dioxide emission. Among these energy sources, biofuels are promising alternative to the petroleum-based fuels. Due to the substantial advantages over plant oils for biofuel production, algae-derived oils have received great interest of both academia and industry and been considered as the next-generation biodiesel feedstock with the potential to meet the existing demand for transportation uses [1-4]. During past decades, substantial progress has been achieved in the exploration of algal biodiesel, including algae screening and selection, genetic

c) The Author(s) 2021. This article is licensed under a Creative Commons Attribution 4.0 International License, which permits use, sharing, adaptation, distribution and reproduction in any medium or format, as long as you give appropriate credit to the original author(s) and the source, provide a link to the Creative Commons licence, and indicate if changes were made. The images or other third party material in this article are included in the article's Creative Commons licence, unless indicated otherwise in a credit line to the material. If material is not included in the article's Creative Commons licence and your intended use is not permitted by statutory regulation or exceeds the permitted use, you will need to obtain permission directly from the copyright holder. To view a copy of this licence, visit http://creativeco mmons.org/licenses/by/4.0/. The Creative Commons Public Domain Dedication waiver (http://creativecommons.org/publicdomain/ zero/1.0/) applies to the data made available in this article, unless otherwise stated in a credit line to the data. 
engineering for trait improvements, and development of technologies for algal cultivation and downstream processes $[5,6]$. Nevertheless, to bring down the production cost and realize the commercialization of algal biodiesel, significant challenges remain to be addressed.

In addition to the neutral lipid triacylglycerol (TAG) that is ideal for making biodiesel, algae are able to produce a broad range of value-added compounds, such as high-quality protein, polyunsaturated fatty acids and carotenoids depending on algae species [7-9]. The coproduction of these high-value compounds with oils from algae has the potential to add benefits and thus offset the algal biodiesel production cost. Astaxanthin, a secondary keto-carotenoid with the highest antioxidant activity found in nature, is high in price and has been widely explored for food, feed, nutraceutical, and pharmaceutical uses [10-12]. Like TAG, astaxanthin is synthesized and accumulated in certain algae under abiotic stress conditions [13-21]. The characteristic of concurrent accumulation of TAG and astaxanthin makes it feasible to employ algae for integrated production of the two compounds.

Chromochloris zofingiensis belongs to green algae and is able to grow robustly to achieve high cell densities under photoautotrophic, heterotrophic and mixotrophic conditions [19, 22-29]. Because of the great capacity in synthesizing TAG (up to $50 \%$ of dry weight) under multiple trophic conditions, C. zofingiensis is considered as a promising feedstock for biodiesel production $[13,17$, $19,28,30]$. This alga can also synthesize astaxanthin at a volumetric level comparable to that Haematococcus pluvialis achieves and has been proposed to serve as an alternative producer of natural astaxanthin $[25,27]$. The robust performance in growth and simultaneous accumulation of TAG and astaxanthin in lipid droplets (LDs) enable C. zofingiensis an appealing alga for production uses [13, 19, 29, 31, 32]. Recently, the chromosome-level genome sequence of $C$. zofingiensis has been released [33], which, together with the workable genetic tools and random mutagenesis for screening target mutants [34-36], provide unprecedented opportunities to better understand the molecular mechanisms for lipid metabolism and carotenogenesis and the crosstalk between TAG and astaxanthin biosynthetic pathways [14, 18, 37-41]. The review centers around $C$. zofingiensis with an aim to (1) summarize recent progress on TAG and astaxanthin production, (2) update molecular understanding of lipid metabolism, carotenogenesis and the communications between TAG and astaxanthin biosynthesis, and (3) discuss engineering strategies for improving the synthesis of either TAG, astaxanthin or both. Efforts made and underway will turn C. zofingiensis into not only a production strain of industrial interest but also an emerging model for fundamental studies on lipid metabolism and carotenogenesis.

\section{Taxonomy, morphology and ultrastructure of $C$. zofingiensis}

C. zofingiensis is a freshwater green alga and has a complicated taxonomic history. It was isolated in 1934 by Dönz and was originally assigned to the Genus Chlorella [42]. Based on detailed observations of morphology and life cycle, Hindák claimed that $C$. zofingiensis was more similar to Muriella aurantiaca than to the Chlorella type species Chlorella vulgaris and thus was recommended to be assigned under the Genus Muriella [43]. Afterwards, the taxonomy of this alga was reconsidered and placed under the Genus Mychonastes based on scanning and transmission electron microscope observations [44]. Nevertheless, the phylogenetic analyses using genetic sequences, such as the nuclear small subunit (18S) rRNA and/or the nuclear ribosomal internal transcribed spacer 2 (ITS2), suggested that C. zofingiensis is distinct from either Chlorella [45], Muriella [46] or Mychonastes [47]. To resolve the uncertain phylogenetic position of C. zofingiensis, Fučíková and his co-worker adopted both morphologic observations and genetic sequences of $18 \mathrm{~S}$ rRNA, ITS2, the large subunit of ribulose 1,5-bisphosphate carboxylase/oxygenase ( $\mathrm{rbcL}$ ) and the plastid-encoded elongation factor TU (tufA), and put C. zofingiensis together with Bracteacoccus cinnabarinus and Bracteacoccus minutus under the genus Chromochloris [48]. A phylogenetic tree based on the 18S rRNA sequences is shown in Fig. 1; although in the same Class Chlorophyceae, $C$. zofingiensis is somewhat distant from the other astaxanthin-producing alga $H$. pluvialis.

C. zofingiensis cells are in unicellular and spherical form without flagellum and the cell size in diameter normally ranges from 2 to $15 \mu \mathrm{m}$ depending on the growth conditions and stages [49]. C. zofingiensis is a haploid alga and can reproduce itself via asexual multiple fission. Sexual reproduction has never been observed in this alga. The life cycle of $C$. zofingiensis is simple and generally involves three phases of growth, ripening, and division (Fig. 2). The multiple fission cell cycle of C. zofingiensis, resembling Scenedesmus and Desmodesmus, is in the consecutive pattern, under which DNA replication and nuclear division are executed multiple times prior to cell division [50]. Therefore, polynuclear cells are observed for $C$. zofingiensis and the number of nucleus within a cell is determined by the number of DNA replication and nuclear division events before cell division. When the parental cell wall ruptures, autospores (up to 32) are released spontaneously and enter into the next multiple fission cell cycle [50]. By contrast, C. reinhardtii has a clustered pattern of multiple fission cell cycle, under 


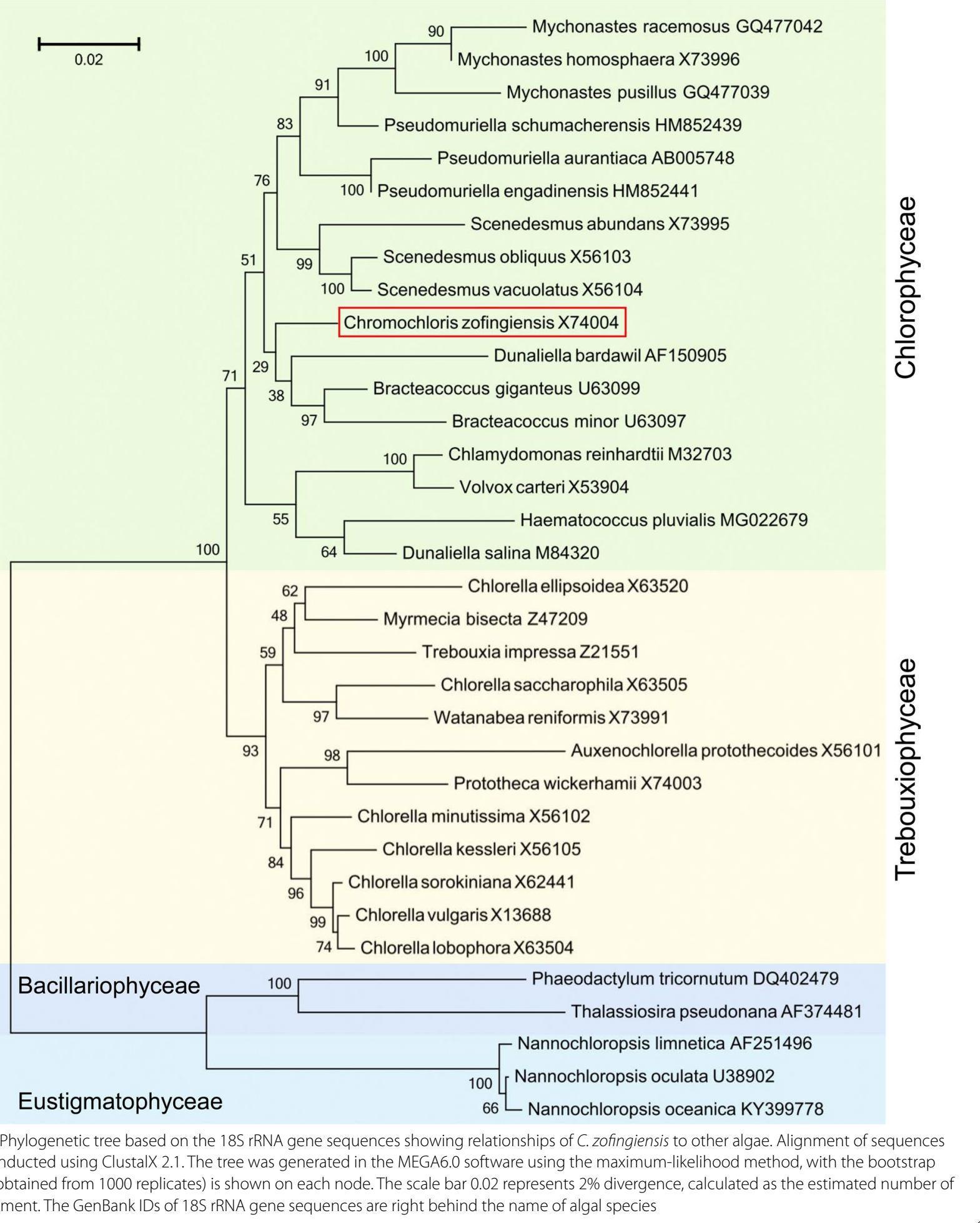

which cell division occurs right after nuclear division; therefore, C. reinhardtii generally does not include polynuclear stages [51].
C. zofingiensis possesses a rigid cell wall, which is mainly composed of glucose and mannose and tends to get thicker under stress conditions [52-55] (Fig. 3). C. 


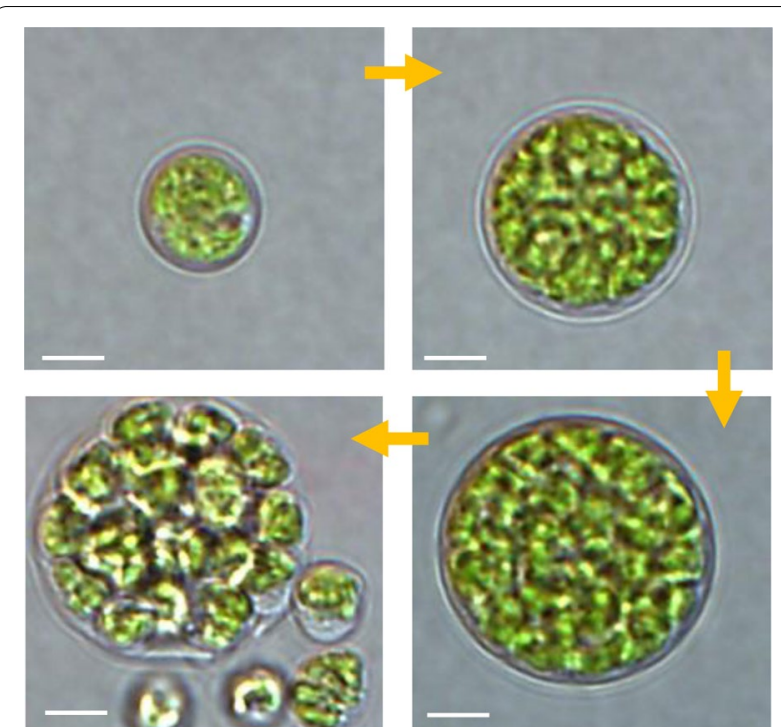

Fig. 2 Light microscopic observation of C. zofingiensis cells under different growth stages. Bar, 2 um

zofingiensis cells appear green under favorable growth conditions and turn orange under stress conditions (Fig. 3), due to the induction of secondary carotenoids including astaxanthin $[13,19,22,54,55]$. Observations based on transmission electron microscopy suggest that C. zofingiensis has a cup-shaped chloroplast sitting peripherally in the cytoplasm, which contains no pyrenoid but scattered starch granules; small LDs are also present and closely associated with the chloroplast (Fig. 3). Stress conditions severely impact the ultrastructure of $C$. zofingiensis cells, leading to the shrunken chloroplast, decreased starch granules and expanded LDs that embrace the chloroplast (Fig. 3). The close proximity of the keto-carotenoids-containing LDs to the cell wall indicates that secondary carotenoids may serve as substrates for synthesizing sporopollenin in cell walls, as is the case in other astaxanthin-producing algae $[55,56]$.

\section{C. zofingiensis as a promising producer of lipids and carotenoids}

\section{Growth physiology and trophic modes}

C. zofingiensis requires certain nutrients to support its growth, including carbon, nitrogen, phosphorus, and inorganic salts. Carbon is the most prominent element and accounts for approximately $50 \%$ of the algal biomass. C. zofingiensis is able to utilize both inorganic and organic carbon sources. Carbon dioxide $\left(\mathrm{CO}_{2}\right)$ is the primary inorganic carbon source for algal growth and it has been reported that some algae can tolerate high $\mathrm{CO}_{2}$ level of $\sim 40 \%$ [1]. There is no report about the tolerance ability of $C$. zofingiensis to $\mathrm{CO}_{2}$ level. In general, a concentration

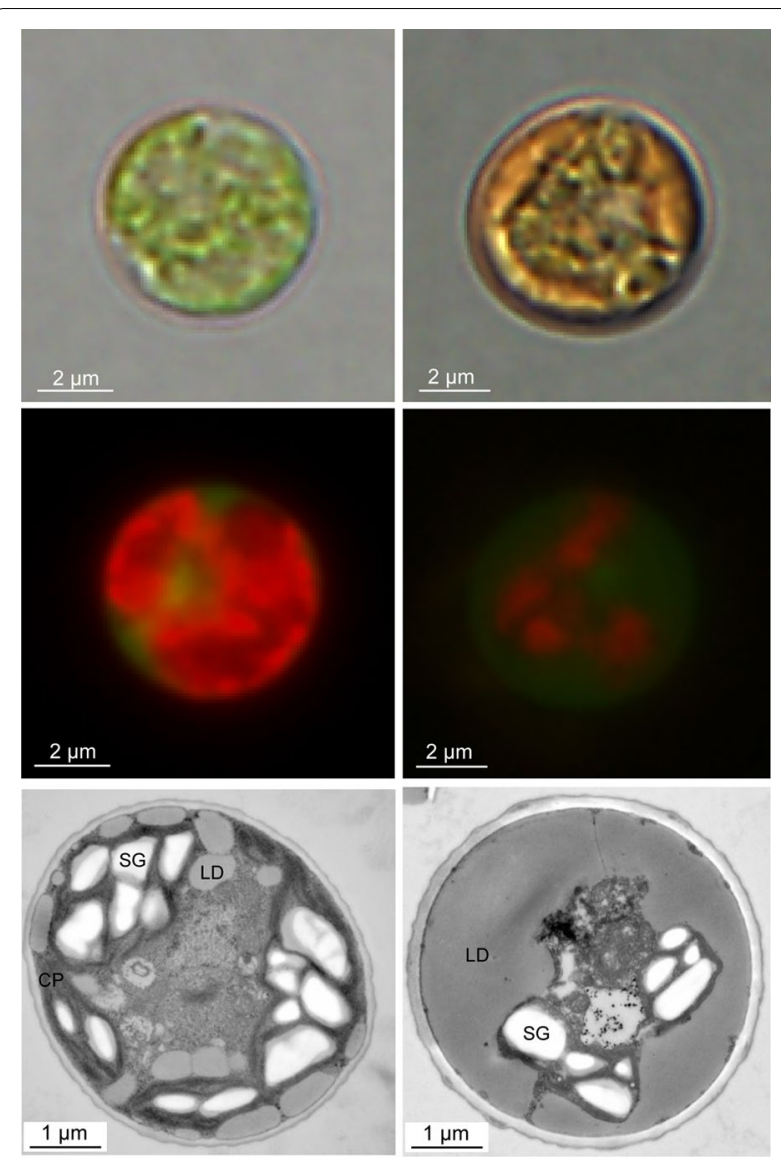

Fig. 3 Microscopic observation of $C$. zofingiensis cells under favorable (left) and stress (right) growth conditions. Up, light microscopy; middle, fluorescent microscopy (red indicates chlorophyll autofluorescence and green indicates neutral lipids stained with BODIPY); bottom, transmission electron microscopy. CP, chloroplast; LD, lipid droplet; SG, starch granule

of $0.5-2 \% \mathrm{CO}_{2}$ (mixed with air by volume) is supplied to sustain photoautotrophic growth of C. zofingiensis, giving rise to a dry biomass density of $\sim 13.5 \mathrm{~g} \mathrm{~L}^{-1}$ in batch cultures $[13,17-20,22,32,55,57,58]$. Light is indispensable for photoautotrophic growth of algae. C. zofingiensis has the ability to maintain its growth under high light intensities $\left(\sim 1500 \mu \mathrm{E} \mathrm{m}^{-2} \mathrm{~s}^{-1}\right)$, suggesting the feasibility of growing this alga outdoors with strong sunlight for mass production [58]. This excellent adaption to high light may be due to the strong non-photochemical quenching ability C. zofingiensis possesses [59]. Within the saturation light range, C. zofingiensis growth is dependent on the light intensity: the higher the light intensity, the greater the biomass achieved $[27,57,58,60]$.

Nitrogen, the important element of protein, is crucial for algal growth. Nitrate, urea and ammonia represent the most commonly used nitrogen sources. C. zofingiensis can utilize both nitrate and urea well for growth, but 
grows poorly with ammonia $[61,62]$. The poor growth is probably due to the acidification of the culture medium resulting from the consumption of ammonia, which has been reported for other algae [28, 63-65]. Nitrogen concentration in the culture medium plays an important role in affecting algal growth. It has been reported that nitrogen limitation/starvation impairs the growth of C. $z o f$ ingiensis severely, accompanied by the enlargement of cell size $[13,17,21,22,41]$. Phosphorus is also an important element required for sustaining algal growth. Nevertheless, phosphorus is less prominent than nitrogen on algal growth and phosphorus limitation/starvation causes only a moderate growth impairment for C. zofingiensis $[8,17]$. It is worth noticing that the micronutrient sulfur has a greater effect than phosphorus on $C$. zofingiensis growth, as suggested by the more severely impaired growth under sulfur starvation compared to under phosphorus starvation [17]. As a freshwater alga, C. zofingiensis is able to tolerate moderate salt levels $(\sim 0.25 \mathrm{M} \mathrm{NaCl})$, yet at the expense of growth [18, 32].

C. zofingiensis can utilize various organic carbon sources, such as sugars, acetate and glycerol for heterotrophic growth, of which glucose is the most widely used one [23, 30, 31]. By contrast, H. pluvialis cannot utilize glucose but acetate for efficient heterotrophic growth [66], probably due to the lack of glucose transporter that is responsible for importing glucose from the medium [67]. In batch cultures, $C$. zofingiensis growth is affected by glucose concentration in the medium, and the final algal biomass yield correlates positively with the initial glucose concentration within the range of $0-30 \mathrm{~g} \mathrm{~L}^{-1}$ $[23,27]$. Nevertheless, high glucose concentration has adverse effect on algal growth. To address this, fed-batch cultivation can be employed, in which glucose is fed into the culture medium time by time to maintain its concentration below a certain level, e.g., $20 \mathrm{~g} \mathrm{~L}^{-1}$, achieving an ultrahigh algal biomass density of $\sim 100 \mathrm{~g} \mathrm{~L}^{-1}$ [25-27, 30, 68]. The ultrahigh fermented C. zofingiensis, with or without dilution, can be used as seed cultures for photoautotrophic growth and carotenogenesis [27, 68]. Furthermore, C. zofingiensis grows well under mixotrophic conditions in the presence of light illumination, where both organic (glucose or acetate) and inorganic carbon sources are provided [21, 24, 29, 62, 69, 70]. It has been proposed that the mixotrophic cultivation has synergistic effect on growth and biomass production of C. zofingiensis [69].

\section{Lipid production}

Lipids can be roughly clarified as polar lipids, e.g., phospholipids and glycolipids that are the main constitutes of various membranes, and neutral lipids, e.g., TAG that is the most energy-dense storage lipid. Under favorable growth conditions, algae contain predominantly polar membrane lipids with only a basal level of TAG; upon stress conditions, algae tend to slow down growth and accumulate TAG in bulk as the carbon and energy reservoir [3]. These stress conditions include but are not restricted to limitation/starvation of nutrients (e.g., nitrogen, phosphorus, sulfur, iron and zinc), high light, salinity, and abnormal temperature [13, 17, 18, 71-78].

The use of $C$. zofingiensis for lipid production has been widely assessed in the past decade $[13,17-20,28$, $30,31,35,60,62,70,79-82]$. Although lipid accumulation in C. zofingiensis has long been observed via transmission electron microscopy [55], lipid quantification of this alga was not performed until 2010 by Liu and his coworkers [30]. This pioneering work examined the effect of various sugars (lactose, galactose, sucrose, fructose, mannose and glucose) on lipid production by heterotrophic C. zofingiensis and found that glucose is superior to other sugars for lipid content and yield. The lipid content in C. zofingiensis reached $\sim 52 \%$ of dry weight, of which TAG accounted for $72 \%$. Fed-batch cultivation was also conducted for $C$. zofingiensis, giving rise to 20.7 $\mathrm{g} \mathrm{L}^{-1}$ and $1.38 \mathrm{~g} \mathrm{~L}^{-1} \mathrm{~d}^{-1}$ for lipid yield and productivity, respectively. Nevertheless, the need of glucose makes lipid production from $C$. zofingiensis less economically viable, particularly for making the low-value commodity biodiesel, driving the exploration of such alternative and cheap carbon sources from cellulosic materials and industrial waste sugars [83-85]. Liu et al. [31] assessed the use of cane molasses, a waste of the sugar industry, for heterotrophic lipid production by C. zofingiensis. The results suggested that cane molasses, after proper pretreatment, could be used as a substitute of glucose to support $C$. zofingiensis for achieving high biomass and lipid productivities. It is worth noting that the sugar-to-lipid conversion ratio is generally below $25 \%$ for heterotrophic C. zofingiensis cultures [30,31, 79], raising the challenge regarding how to improve the sugar-based lipid yield.

Concerning photoautotrophic lipid production, Mulders et al. [19] assessed C. zofingiensis cultures under nitrogen deprivation (ND) conditions, in which TAG content and yield reached $0.34 \mathrm{~g} \mathrm{mg}^{-1}$ dry weight and 2.9 $\mathrm{g} \mathrm{L}^{-1}$, respectively. Later, Liu et al. [13] compared lipid production performance by photoautotrophic C. zofingiensis under various conditions of ND, high light (HL) and the combination of ND and HL (ND + HL). ND + HL enabled C. zofingiensis to produce the highest levels of total lipids and TAG, followed by ND and HL. Nevertheless, due to the compromised biomass production, TAG productivities achieved under ND and ND + HL conditions were lower than that under HL conditions. To promote TAG productivity, the authors employed a nitrogen limitation strategy coupled with a semi-continuous 
culture system. The effect of other nutrients, such as phosphorus and sulfur, was also evaluated for C. zofingiensis: similar to ND, sulfur deprivation (SD) induced TAG accumulation yet less prominent; by contrast, phosphorus deprivation (PD) showed little impact on TAG synthesis [17]. Interestingly, other algae, such as Nannochloropsis and Phaeodactylum, are vulnerable to PD for TAG induction [74, 86, 87], highlighting the evolutionary divergence of these algae in sensing and responding to phosphorus changes. C. zofingiensis is able to grow in the presence of moderate salinity levels $[18,22,88]$. As shown in other green algae [89-92], C. zofingiensis was reported to synthesize and accumulate TAG upon salinity stress (SS) [18], pointing to the potential of using this alga for lipid production under saline environment, thus reducing freshwater footprint. Furthermore, the combination of HL and SS (HL + SS) was shown to induce more TAG in C. zofingiensis and give rise to higher TAG yield and productivity than HL and SS alone did [32]. In addition, it has been recently reported that lipid accumulation in C. zofingiensis could be stimulated by certain phytohormones, resulting in enhanced lipid yield and productivity [29]. A summary of lipid production by $C$. zofingiensis under various conditions is listed in Table 1. There are a number of reviews about lipid production by microalgae during the past decades; the lipid content and lipid productivity, depending on microalgal species/ strains and culture conditions, normally range from 20 to $60 \%$ of dry weight and 30 to $600 \mathrm{mg} \mathrm{L}^{-1} \mathrm{~d}^{-1}$, respectively [3, 93-95]. It may be not appropriate to conclude by direct comparison of lipid content and productivity between $C$. zofingiensis and other algae, as the culture conditions are different. Nevertheless, the TAG content ( $\sim 48 \%$ of dry weight), yield $\left(\sim 20.4 \mathrm{~g} \mathrm{~L}^{-1}\right)$ and productivity $\left(\sim 1.4 \mathrm{~g} \mathrm{~L}^{-1}\right.$ day $\left.^{-1}\right)$ achieved for $C$. zofingiensis are overall comparable to or even higher than those from other commonly studied and potential lipid production algae, such as Chlorella, Scenedesmus, Nannochloropsis, etc. [28, 94, 96-99].

The fatty acid composition of lipids is also important, as it determines key properties of biodiesel, such as cetane number, heat of combustion, oxidative stability, cloud point, lubricity [100]. Similar to plant oils, C. zofingiensis lipids consist predominantly of fatty acids in the length of 16-18 carbons [30]. The relative abundance of fatty acids in C. zofingiensis varies largely depending on the culture conditions [13, 17, 18, 28, 29, 31, 62, 79]. In general, saturated fatty acids provide oxidative stability, while unsaturated fatty acids benefit low-temperature stability. It is believed that oleic acid $\left(\mathrm{C} 18: 1^{\Delta 9}\right)$ can serve

Table 1 Summary of TAG and astaxanthin production by C. zofingiensis

\begin{tabular}{|c|c|c|c|c|c|c|c|c|c|}
\hline \multirow[t]{2}{*}{ References } & \multirow[t]{2}{*}{ Culture conditions } & \multicolumn{2}{|l|}{ Biomass } & \multicolumn{3}{|l|}{ TAG or TFA } & \multicolumn{3}{|l|}{ Astaxanthin } \\
\hline & & $\begin{array}{l}\text { Concentration } \\
\left(\mathrm{g} \mathrm{L}^{-1}\right)\end{array}$ & $\begin{array}{l}\text { Productivity } \\
\left(\mathrm{g} \mathrm{L}^{-1} \text { day }^{-1}\right)\end{array}$ & $\begin{array}{l}\text { Content } \\
\left(\text { g g }^{-1} \mathrm{DW}\right)\end{array}$ & $\begin{array}{l}\text { Yield } \\
\left(\mathrm{g} \mathrm{L}^{-1}\right)\end{array}$ & $\begin{array}{l}\text { Productivity } \\
\left(\mathrm{g} \mathrm{L}^{-1} \text { day }^{-1}\right)\end{array}$ & $\begin{array}{l}\text { Content } \\
\left(\mathrm{mg} \mathrm{g}^{-1} \mathrm{DW}\right)\end{array}$ & $\begin{array}{l}\text { Yield } \\
\left(\mathrm{mg} \mathrm{L}^{-1}\right)\end{array}$ & $\begin{array}{l}\text { Productivity } \\
\left(\mathrm{mg} \mathrm{L}^{-1} \text { day }^{-1}\right)\end{array}$ \\
\hline [88] & P, Batch & - & - & - & - & - & 6.8 & - & 0.8 \\
\hline [22] & P, Batch & 7.0 & 0.7 & - & - & - & 3.7 & 25.0 & 1.3 \\
\hline [24] & M, Batch & 9.5 & - & - & - & - & 1.3 & 12.5 & - \\
\hline [26] & $\mathrm{H}, \mathrm{FB}$ & 53.0 & 3.3 & - & - & - & 0.7 & 32.4 & 2.0 \\
\hline [30] & $\mathrm{H}, \mathrm{FB}$ & 43.1 & 2.9 & $0.48^{\mathrm{a}}$ & 20.7 & 1.4 & - & - & - \\
\hline [31] & $\mathrm{H}$, Batch & 12.9 & 1.6 & 0.33 & 4.2 & 0.5 & 1.2 & 13.6 & 1.7 \\
\hline [25] & $\mathrm{H}, \mathrm{FB}$ & 45.6 & 4.7 & - & - & - & 1.2 & 56.1 & 5.6 \\
\hline [77] & M, Batch & 11.9 & 0.6 & $0.42^{\mathrm{a}}$ & 5.0 & 0.3 & 2.2 & 25.8 & 1.3 \\
\hline [19] & P, Batch & 8.2 & 0.6 & 0.34 & 2.8 & 0.3 & 2.4 & 19.6 & 1.4 \\
\hline [13] & $\begin{array}{l}\text { P, Batch } \\
\text { P, SC }\end{array}$ & $\begin{array}{l}7.5 \\
-\end{array}$ & $\begin{array}{l}1.4 \\
1.0\end{array}$ & $\begin{array}{l}0.39 \\
0.29\end{array}$ & $\begin{array}{l}1.5 \\
-\end{array}$ & $\begin{array}{l}0.2 \\
0.3\end{array}$ & $\begin{array}{l}4.9 \\
3.2\end{array}$ & $\begin{array}{l}12.8 \\
-\end{array}$ & $\begin{array}{l}2.0 \\
3.3\end{array}$ \\
\hline [21] & M, Batch & 6.0 & 0.5 & - & - & - & 6.5 & 38.9 & 3.2 \\
\hline [68] & $\mathrm{M}, \mathrm{TS}$ & 98.4 & 7.0 & - & - & - & 0.8 & 73.3 & 5.2 \\
\hline [17] & P, Batch & 1.8 & 0.2 & 0.27 & 0.4 & 0.1 & 3.9 & 4.5 & 0.6 \\
\hline [27] & $\begin{array}{l}\mathrm{H}, \mathrm{FB} \\
\mathrm{H}-\mathrm{P}, \mathrm{TS}\end{array}$ & $\begin{array}{l}71.1 \\
73.7\end{array}$ & $\begin{array}{l}5.8 \\
4.8\end{array}$ & $\begin{array}{l}- \\
-\end{array}$ & $\begin{array}{l}- \\
-\end{array}$ & $\begin{array}{l}- \\
-\end{array}$ & $\begin{array}{l}0.7 \\
2.7\end{array}$ & $\begin{array}{l}47.3 \\
194.5\end{array}$ & $\begin{array}{l}4.0 \\
9.9\end{array}$ \\
\hline [29] & M, Batch & 8.3 & 0.7 & $0.65^{\mathrm{a}}$ & 5.4 & 0.5 & 13.1 & 89.9 & 7.5 \\
\hline [18] & P, Batch & 3.8 & 1.0 & 0.19 & 0.5 & 0.1 & 3.0 & 6.8 & 1.7 \\
\hline [32] & P, Batch & 7.2 & 1.1 & $0.42^{\mathrm{a}}$ & 3.0 & 0.5 & 6.0 & 41.8 & 7.0 \\
\hline [70] & $\mathrm{M}, \mathrm{FB}$ & 7.8 & 1.1 & $0.42^{\mathrm{a}}$ & - & 0.46 & - & - & 2.0 \\
\hline
\end{tabular}

${ }^{a}$ TFA; $\mathrm{P}$, photoautotrophic culture; $\mathrm{M}$, mixotrophic culture; $\mathrm{H}$, heterotrophic culture; SC, semi-continuous culture; FB, fed-batch culture; TS, two-stage cultivation 
as a balance between oxidative stability and low-temperature performance, and its high abundance is beneficial to biodiesel quality $[100,101]$. In C. zofingiensis, C18:1 ${ }^{\Delta 9}$ abundance correlates positively with TAG content and its relative abundance in TAG can reach $\sim 60 \%[13,17,18$, 30,31 , pointing to the potential of using lipids from this alga for making high-quality biodiesel.

\section{Carotenoid production}

Carotenoids, the abundant natural pigments, are widely distributed in photosynthetic organisms, some nonphotosynthetic bacteria and fungi [102]. The common carotenoids found in vascular plants, e.g., $\beta$-carotene, zeaxanthin, neoxanthin, antheraxanthin, violaxanthin, $\alpha$-carotene and lutein, are also present in green algae. These primary carotenoids serve as important components of photosynthetic apparatus and are critical for photoautotrophic growth. Aside from primary carotenoids, some green algae synthesize keto-carotenoids (also called secondary carotenoids), such as echinenone, canthaxanthin, adonirubin, adonixanthin, astaxanthin and keto-lutein [8, 54, 55, 88, 103-107]. Distinct from primary carotenoids, secondary carotenoids are synthesized in large quantities by certain algae only under specific stress conditions and generally reside in the extrachloroplastic organelle lipid body (LD) $[40,55,108,109]$. Among the secondary carotenoids, astaxanthin possesses the strongest antioxidant activity with broad applications and has long been receiving interests of both academia and industry $[10,56,110,111]$. So far, $H$. pluvialis is the only alga used for commercial production of astaxanthin. Nevertheless, slow growth rate, low biomass production and ease of contamination by other fast-growing organisms restrict the yield of astaxanthin from $H$. pluvialis, driving the exploration of alternative algal producers, e.g., C. zofingiensis [8].

In addition to astaxanthin, $C$. zofingiensis synthesizes a series of other keto-carotenoids including echinenone, canthaxanthin, adonixanthin and keto-lutein [8, 107]. Astaxanthin production from photoautotrophic C. zofingiensis cultures has long been studied [54, 55, 104, $112]$. In these early works, the only recorded secondary carotenoids were astaxanthin $(\sim 70 \%)$ and canthaxanthin ( 30\%). Later, Del Campo et al. [22] evaluated the effect of different environmental and nutritional factors (i.e., temperature, light intensity, salinity level and nitrate concentration) on astaxanthin production by C. zofingiensis and achieved a maximum astaxanthin yield of $25 \mathrm{mg} \mathrm{L}^{-1}$ and productivity of $1.3 \mathrm{mg} \mathrm{L}^{-1}$ day $^{-1}$. In the study conducted by Mulders et al. [19], the ND-induced C. $z$ ofingiensis accumulated astaxanthin, canthaxanthin, and keto-lutein as the main secondary carotenoids; the astaxanthin content, yield and productivity acheieved were
$2.4 \mathrm{mg} \mathrm{g}^{-1}$ dry weight, $20 \mathrm{mg} \mathrm{L}^{-1}$ and $1.4 \mathrm{mg} \mathrm{L}^{-1}$ day $^{-1}$, respectively. Comparatively, among the three nutrient stress conditions of ND, PD and SD, ND enabled $C$. zofingiensis to synthesize the highest level of astaxathin (3.9 $\mathrm{mg} \mathrm{g}^{-1}$ dry weight), followed by SD and PD [17]. The effect of stress conditions alone or in combination on astaxanthin production by $C$. zofingiensis has also been comparatively examined [13, 32]. Apparently, ND+HL was demonstrated to be more efficient than ND or HL alone for astaxanthin induction in C. zofingiensis, giving rise to an astaxanthin content of $4.9 \mathrm{mg} \mathrm{g}^{-1}$ dry weight in a 6-day batch culture [13]. Nevertheless, the astaxanthin productivity was compromised by the impaired growth under ND+ HL and thus just comparable to that under HL (2.0 versus $1.8 \mathrm{mg} \mathrm{L}^{-1}$ day $^{-1}$ ) [13]. Similarly, HL + SS was shown to surpass HL or SS alone in inducing astaxanthin synthesis and allowed C. zofingiensis to accumulate astaxanthin at a level of $\sim 6.0 \mathrm{mg} \mathrm{g}^{-1}$ dry weight [32]. Unlike ND+ HL, HL + SS was also superior to HL or SS alone and gave rise to the greatest astaxanthin yield (41.8 $\left.\mathrm{mg} \mathrm{L}^{-1}\right)$ and productivity (7.0 $\mathrm{mg} \mathrm{L}^{-1}$ day $^{-1}$ ) [32]. Astaxanthin content in C. zofingiensis could be further promoted to $6.8 \mathrm{mg} \mathrm{g}^{-1}$ dry weight under the combination of three stress conditions, i.e., HL, ND and SS, yet astaxanthin productivity was low $\left(0.8 \mathrm{mg} \mathrm{L}^{-1}\right.$ day $\left.^{-1}\right)$ because of the severely impaired growth [88].

Heterotrophic production of astaxanthin from C. zofingiensis has also been intensively studied, using sugars particularly glucose as the sole carbon and energy source [23, 25-27, 31, 68, 113, 114]. Concerning heterotrophic C. zofingiensis cultures, sugar concentration or carbon/ nitrogen $(\mathrm{C} / \mathrm{N})$ ratio in the culture medium correlates with astaxanthin content in the alga, e.g., as sugar concentration increased from $5 \mathrm{~g} \mathrm{~L}^{-1}$ to $50 \mathrm{~g} \mathrm{~L}^{-1}$, astaxanthin content rose from 0.44 to $1.01 \mathrm{mg} \mathrm{g}^{-1}$ dry weight [23]. Reactive oxygen species and reactive nitrogen species were shown to promote astaxanthin accumulation in heterotrophic C. zofingiensis cells [113, 114]. Of six sugars tested, glucose and mannose were more effective than other four for inducing astaxanthin accumulation in $C$. zofingiensis batch cultures; using the glucose-based fedbatch cultivation (15-day period), biomass concentration and astaxnathin yield increased from $10.3 \mathrm{~g} \mathrm{~L}^{-1}$ and 10.5 $\mathrm{mg} \mathrm{L}^{-1}$ to $51.8 \mathrm{~g} \mathrm{~L}^{-1}$ and $32.4 \mathrm{mg} \mathrm{L}^{-1}$, respectively [26]. Later, the fed-batch cultivation of $C$. zofingiensis using pretreated molasses was performed, in which astaxanthin yield and productivity after 10 days of cultivation reached $45.6 \mathrm{mg} \mathrm{L}^{-1}$ and $5.35 \mathrm{mg} \mathrm{L}^{-1}$ day $^{-1}$, respectively [25]. In another fed-batch fermentation study (14-day period), the authors reported even higher biomass concentration and astaxanthin yield, which were $98.4 \mathrm{~g} \mathrm{~L}^{-1}$ and $73.3 \mathrm{mg} \mathrm{L}^{-1}$, respectively [68]. Albeit with ultrahigh biomass concentration, these heterotrophic $C$. zofingiensis 
cultures contained astaxanthin below $1.0 \mathrm{mg} \mathrm{g}^{-1}$ dry weight $[25,26,68]$, much less than that achieved in photoautotrohphic cultures [13,17, 19, 32, 88]. Likely, light is a key inducer for enhancing astaxanthin accumulation in C. zofingiensis. In this context, Sun et al. [27] developed a novel heterotrophy-photoinduction culture strategy for C. zofingiensis: the alga was first cultured in a heterotrophic fed-batch mode for achieving ultrahigh biomass density, followed by transfer of the heterotrophic cultures without dilution to light for photoinduction of astaxanthin. This strategy enabled C. zofingiensis to produce 2.6 $\mathrm{mg} \mathrm{g}^{-1}$ astaxanthin and so far the highest astaxanthin yield and productivity, i.e., $194.5 \mathrm{mg} \mathrm{L}^{-1}$ and $9.9 \mathrm{mg} \mathrm{L}^{-1}$ day $^{-1}$.

There have been several reports about using mixotrophic C. zofingiensis cultures for astaxanthin production $[21,24,29,77]$. In the study conducted by Chen et al. [21], C. zofingiensis was cultured with a high $\mathrm{C} / \mathrm{N}$ ratio in the presence of $\mathrm{HL}$, and astaxanthin content, yield and productivity achieved were $6.5 \mathrm{mg} \mathrm{g}^{-1}, 38.9 \mathrm{mg} \mathrm{L}^{-1}$ and $3.24 \mathrm{mg} \mathrm{L}^{-1} \mathrm{day}^{-1}$, respectively. It has been suggested that phytohormones can be employed in combination with stress conditions to enhance astaxanthin accumulation in H. pluvialis [115]. Similarly, certain phytohormones were shown to promote astaxanthin production by $C$. zofingiensis under mixotrophic growth conditions, with astaxanthin content, yield and productivity being $13.1 \mathrm{mg} \mathrm{g}^{-1}, 89.9 \mathrm{mg} \mathrm{L}^{-1}$ and $7.49 \mathrm{mg} \mathrm{L}^{-1}$ day $^{-1}$, respectively [29]. The detailed summary of astaxanthin production by $C$. zofingiensis under various conditions is listed in Table 1. Albeit the highest astaxanthin content obtained for C. zofingiensis (13.1 $\mathrm{mg} \mathrm{g}^{-1}$ dry weight) is still much lower than that for $H$. pluvialis $\left(>40 \mathrm{mg} \mathrm{g}^{-1}\right.$ dry weight), the astaxanthin yield $\left(\sim 194.5 \mathrm{mg} \mathrm{L}^{-1}\right)$ and productivity $\left(\sim 9.9 \mathrm{mg} \mathrm{L}^{-1}\right.$ day $\left.^{-1}\right)$ for C. zofingiensis are comparable to and in some cases higher than that of $H$. pluvialis [116-121].

Natural astaxanthin has free and esterified forms. Astaxanthin-producing algae, with a couple of exceptions that produce only free form $[105,122]$, accumulate both forms and the relative proportions depend on the algae species and culture conditions $[8,56,104]$. It has been suggested that esterified astaxanthin is more stable and has stronger antioxidant ability than free astaxanthin $[123,124]$. C. zofingiensis accumulates esterified astaxanthin as the major proportion, which can reach $~ 92 \%$ of total astaxanthin and $\sim 70 \%$ of total secondary carotenoids under induction conditions $[13,14,17,32,55,104$, 107].

\section{Simultaneous production of TAG and astaxanthin}

It is believed that integrated production of TAG with high-value products from algae has the potential to improve algal biodiesel production economics [7]. The implementation of this concept, from a biorefinery point of view, requires simultaneous accumulation of TAG and high-value products in algae. The high-value carotenoid astaxanthin, similar to TAG, belongs to secondary metabolites and is stored in LDs in algae [40, 109]. In C. zofingiensis both TAG and astaxanthin are induced to synthesize and accumulate under certain above-mentioned conditions, such as ND, SD, HL, SS, ND+HL, HL + SS, high sugar concentration $[13,14,17-19,29,31$, 32, 62]. Specifically, when plotting TAG contents with astaxanthin contents from different time points of each condition, a strong linear relationship was observed with the $R^{2}$ being over $0.975[13,14]$. This reflects the coordinated and simultaneous accumulation of TAG and astaxanthin in C. zofingiensis and guarantees the feasibility of using this alga for integrated production of the two compounds. In this context, C. zofingiensis has the potential to serve as a leading algal producer of lipids for biodiesel and an alternative promising source of natural astaxanthin.

\section{Extraction of TAG and astaxanthin}

Considering that both TAG and astaxanthin are stored in LDs of C. zofingiensis [40], co-extraction of these two compounds from the alga is possible. Nevertheless, $C$. zofingiensis possesses rigid cell wall particularly under stress conditions [8] and thus cell disruption is required to facilitate extraction of TAG and astaxanthin from the alga and downstream processes. Many mechanic and non-mechanic disruption methods have been developed and applied to rupture cell walls of various microalgae; the former include bead beating [125], grinding [126], ultrasonication [127], high-pressure homogenization [128] and expeller pressing [129], and the latter include repeated freeze-thaw [130], osmotic shock [131], microwave radiation [132] and enzymatic digestion [133]. These methods should also work for cell wall disruption of C. zofingiensis, though modifications may be needed due to differences in cell wall composition and rigidity between C. zofingiensis and other algae [134].

Organic solvents can be applied to ruptured algal cells for easy extraction of lipids and pigments. The frequently used organic system for $C$. zofingiensis is a mixture of chloroform and methanol $(2: 1, \mathrm{v} / \mathrm{v})$, which has been demonstrated to extract both TAG and astaxanthin efficiently [13, 14, 17]. Nevertheless, this polar organic mixture extracts not only TAG and astaxanthin but also polar lipids. Low-polarity organic solvents, such as hexane/isopropanol, have been used for highly selective extraction of TAG from microalgae $[135,136]$. This should work for $C$. zofingiensis to selectively extract TAG as well as astaxanthin. As the use of organic solvents 
brings environmental and safety issues, alternative green solvents, such as supercritical fluids (e.g., $\mathrm{CO}_{2}$ ) and ionic liquids, have emerged as the extraction media for lipids from microalgal biomass [137-140]. Whether these methods can be applied to $C$. zofingiensis for efficient TAG and astaxanthin extraction needs to be experimentally evaluated.

\section{Lipid metabolism in C. zofingiensis}

Although the past decade has witnessed substantial progress in lipid production by $C$. zofingiensis, the content and yield need to be improved for more viable biodiesel uses, which rely on genetic modifications of the alga guided by deep understanding of lipid metabolism. The availability of $C$. zofingiensis genome sequence [33] and knowledge from C. reinhardtii, a close relative to C. zofingiensis with detailed study on acyl-lipid metabolism [141-143], accelerate research and understanding on lipogenesis for TAG biosynthesis in C. zofingiensis.

\section{Profiles of fatty acids and glycerolipid classes}

The fatty acid profile of $C$. zofingiensis has been determined and reported by numerous studies in the past decade [13, 17, 18, 28-32, 37, 62, 79]. In general, the fatty acids are composed of C16:0, C16:1 $1^{\Delta 7}, C 16: 1^{\Delta 3 t}$, $\mathrm{C} 16: 2^{\Delta 7,10}, \mathrm{C} 16: 3^{\Delta 7,10,13}, \mathrm{C} 16: 3^{\Delta 4,7,10,13} \mathrm{C} 18: 0, \mathrm{C} 18: 1^{\Delta 9}$, $\mathrm{C} 18: 2^{\Delta 9,12}, \mathrm{C} 18: 3^{\Delta 6,9,12}, \mathrm{C} 18: 3^{\Delta 9,12,15}$, and $\mathrm{C} 18: 4^{\Delta 6,9,12,15}$

(Fig. 4). This differs from the fatty acid composition of C. reinhardtii in which $\mathrm{C} 18: 3^{\Delta 6,9,12}$ and $\mathrm{C} 18: 4^{\Delta 6,9,12,15}$ are replaced by $C 18: 3^{\Delta 5,9,12}$ and $C 18: 4^{\Delta 5,9,12,15}$, respectively [141]. The relative abundance of fatty acids in $C$. zofingiensis varies greatly depending on culture conditions, for example, the major monounsaturated fatty acid $C 18: 1^{\Delta 9}$ has a considerably higher percentage under $\mathrm{ND}+\mathrm{HL}$ than under favorable growth conditions, with a lower percentage of polyunsaturated fatty acids [13].

In addition to the polar glycerolipids present in C. reinhardtii, e.g., monogalactosyl diacylglycerol (MGDG), digalactosyl diacylglycerol (DGDG), sulfoquinovosyl diacylglycerol (SQDG), phosphatidylglycerol (PG), phosphatidylinositol (PI), phosphatidylethanolamine (PE) and diacylglycerol- $N, N, N$-trimethylhomoserine (DGTS), C. zofingiensis contains phosphatidylcholine (PC) as well $[18,37,38]$. As indicated in Fig. 4 based on the data from Liu et al. [37], under nitrogen-replete favorable growth conditions, the lipid fraction accounts for only a small proportion of cell mass, of which membrane lipids particularly the glycolipids MGDG and DGDG are the major lipid classes. By contrast, under such stress condition as ND, the lipid fraction dominates the proportion of cell mass, contributed by the huge increase of TAG. Polar lipids, on the other hand, decrease severely in their proportion.

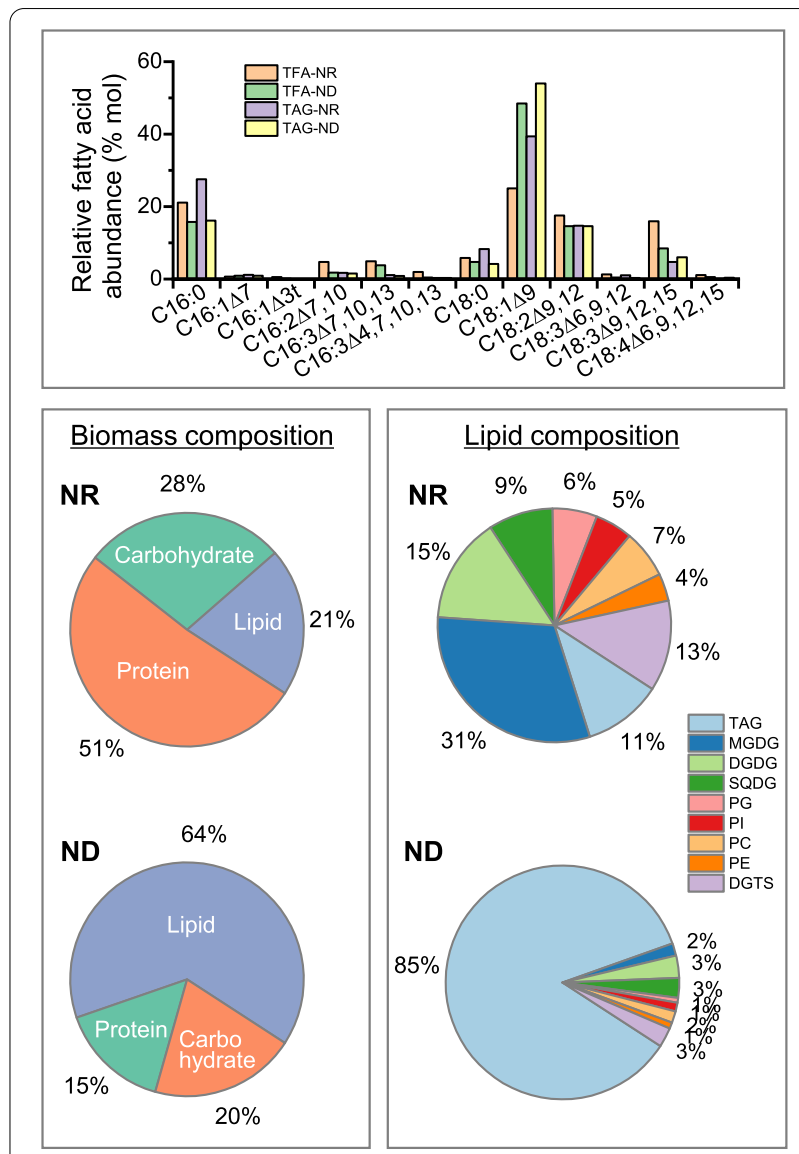

Fig. 4 Profiles of fatty acids and glycerolipids in C. zofingiensis under nitrogen replete (NR) and nitrogen deprivation (ND) conditions. DGDG, digalactosyl diacylglycerol; DGTS, diacylglycerol-N,N,N-tri methylhomoserine; MGDG, monogalactosyl diacylglycerol; SQDG, sulfoquinovosyl diacylglycerol; PE, phosphatidylethanolamine; $P G$, phosphatidylglycerol; PI, phosphatidylinositol; TAG, triacylglycerol; TFA, total fatty acids

\section{Fatty acid biosynthesis, desaturation and degradation}

Green algae, similar to vascular plants, perform de novo fatty acid synthesis in the chloroplast, using acetyl-CoA as the precursor and building block [141]. Multiple routes are proposed for producing acetyl-CoA: from pyruvate mediated by pyruvate dehydrogenase complex (PDHC), from pyruvate via PDHC bypass, from citrate through the ATP-citrate lyase (ACL) reaction, and from acetylcarnitine via carnitine acetyltransferase reaction [144]. C. zofingiensis genome harbors genes encoding enzymes involved in the first three routes [37]. Taking into account the predicted subcellular localization information and transcriptomics data [18, 37, 38], C. zofingiensis likely employs both PDHC and PDHC bypass routes, but mainly the former one, to supply acetyl-CoA in the chloroplast for fatty acid synthesis.

De novo fatty acid synthesis in the chloroplast consists of a series of enzymatic steps mediated by acetyl-CoA 
carboxylase (ACCase), malonyl-CoA:acyl carrier protein (ACP) transacylase (MCT), and type II fatty acid synthase (FAS), an easily dissociable multisubunit complex (Fig. 5). The formation of malonyl-CoA from acetyl-CoA, a committed step in fatty acid synthesis, is catalyzed by ACCase [145]. The chloroplast-localized ACCase in C. zofingiensis is a tetrasubunit enzyme consisting of $\alpha$-carboxyltransferase, $\beta$-carboxyltransferase, biotin carboxyl carrier protein, and biotin carboxylase.
These subunits are well correlated at the transcriptional level $[18,33,37,39]$. Malonyl-CoA has to be converted to malonyl-acyl carrier protein (ACP), through the action of $\mathrm{MCT}$, before entering the subsequent condensation reactions for acyl chain extension. The condensation reactions are catalyzed by three types of 3-ketoacyl-ACP synthase (KAS): KAS III catalyzes the first condensation to form C4:0-ACP from malonyl-ACP and acetyl-CoA, KAS I catalyzes the subsequent condensation reactions
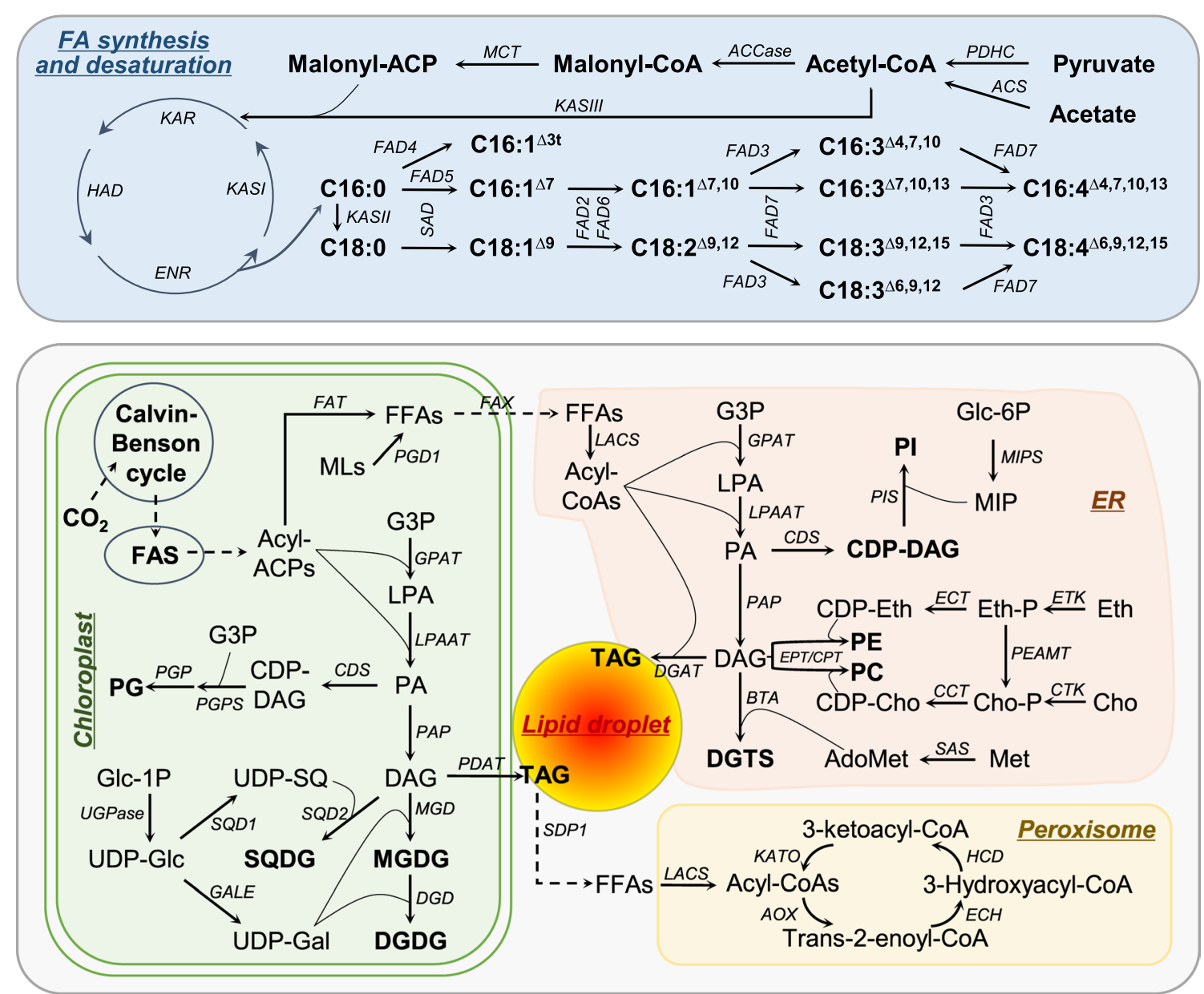

Fig. 5 Lipid metabolic pathways in C. zofingiensis. ACCase, acetyl-CoA carboxylase; AdoMet, S-adenosylmethionine; AOX, acyl-CoA oxidase; BAT, betaine lipid synthase; CDS, phosphatidate cytidylyltransferase; CCT, choline-phosphate cytidylyltransferase; CHK, choline kinase; Cho, Choline; DAG, diacylglycerol; DGAT, Diacylglycerol acyltransferase; DGD, digalactosyldiacylglycerol synthase; DGDG, digalactosyl diacylglycerol; ECH, enoyl-CoA hydratase; ECT, CDP-Ethanolamine synthase; ENR, enoyl-ACP reductase; EPT/CPT, ethanolaminephosphotransferase/cholinephosphotransferase; Eth, Ethanolamine; ETK, ethanolamine kinase; GALE, UDP-galactose 4-epimerase; FAD, fatty acid desaturase; FAT, acyl-ACP thioesterase; G3P, glycerol-3-phosphate; GPAT, glycerol-3-phosphate acyltransferase; HAD, 3-ketoacyl-ACP dehydratase; HCD, 3-hydroxyacyl-CoA dehydrogenase; KAR, 3-ketoacyl-ACP reductase; KAS, 3-ketoacyl-ACP synthase; KATO, 3-ketoacyl-CoA thiolase; LACS, long-chain acyl-CoA synthetase; LPA, lysophosphatidic acid; LPAAT, lysophosphatidic acid acyltransferase; MCT, malonyl-CoA:acyl carrier protein transacylase; Met, methionine; MIPS, myo-inositol-1-phosphate synthase; MGD, monogalactosyldiacylglycerol synthase; MGDG, monogalactosyl diacylglycerol; MLDP, major lipid droplet protein; PA, phosphatidic acid; PAP, phosphatidate phosphatase; PC, phosphatidylcholine; PDAT, phospholipid:diacylglycerol acyltransferase; PE, phosphatidylethanolamine; PEAMT, phosphoethanolamine methyltransferase; PG, phosphatidylglycerol; PGP, phosphatidylglycerophosphatase; PGPS, phosphatidylglycerophosphate synthase; PI, phosphatidylinositol; PIS, phosphatidylinositol synthase; PGD1, Plastid Galactoglycerolipid Degradation1; SAD, stearoyl-ACP desaturase; SAS, S-adenosylmethionine synthase; SQDG, sulfoquinovosyl diacylglycerol; SDP1, Sugar-Dependent1 TAG lipase; TAG, triacyglycerol; UGPase, UDP-glucose pyrophosphorylase 
up to C16:0-ACP, while KAS II catalyzes the formation of C18:0-ACP from C16:0-ACP. Following each condensation, additional reduction and dehydration steps are required to finish the two-carbon addition process, which are mediated in succession by 3-ketoacyl-ACP reductase (KAR), 3-hydroxyacyl-ACP dehydratase (HAD), and enoyl-ACP reductase (ENR) (Fig. 5). C. zofingiensis has been reported to possess one gene copy encoding the chloroplastic form of each KAS I, KAS II, KAS III, KAR, HAD and ENR; these genes are expressed in a well-coordinated manner to allow effective utilization of acetylCoA for the production of C16- and C18-ACPs [37].

Acyl-ACPs in the chloroplast can be either utilized by chloroplast-localized acyltransferases or converted to free fatty acids by the action of acyl-ACP thioesterase (FAT) [141]. Similar to C. reinhardtii, C. zofingiensis harbors a single-copy $F A T$ gene, which correlates well with the de novo fatty acid synthetic genes at the transcriptional levels $[18,37]$. The released free fatty acids, assisted with a fatty acid export 1 (FAX1), are translocated across chloroplast envelopes, which is characterized first in Arabidopsis [146] and then in algae [147, 148]. There are three putative FAX1-encoding genes present in C. zofingiensis [18]. Prior to integration into glycerolipids, the exported free fatty acids need to be ligated with CoA to form acyl-CoAs, catalyzed by long-chain acyl-CoA synthetase (LACS). Similar to vascular plants, such as Arabidopsis [149], algae possess multiple copies of putative $L A C S$ genes, e.g., three in C. reinhardtii [150], six in $C$. zofingiensis [151], five in Phaeodactylum tricornutum [152], and eight in Thalassiosira pseudonana [153]. Of the six C. zofingiensis LACS members, CzLACS2 through CzLACS5 are bona fide LACS enzymes and have overlapping yet distinct substrate preferences [151]. Considering the transcriptional expression data and subcellular localization results, CzLACS2 through CzLACS4, residing at endoplasmic reticulum (ER), are likely involved in TAG biosynthesis, while the peroxisome-localized CzLACS5 participates in fatty acid $\beta$-oxidation process [151].

In C. zofingiensis, unsaturated fatty acids dominate over saturated fatty acids (Fig. 4). The synthesis of unsaturated fatty acids involves a series of desaturases. Aside from the chloroplast-localized stearoyl-ACP desaturase (SAD) that is soluble and utilizes C18:0-ACP as substrate to form C18:1 $1^{\Delta 9}$-ACP [154], fatty acid desaturases (FADs) are usually membrane-bound and act on complex lipids for desaturation $[141,155]$. C. zofingiensis contains two copies of $S A D$ genes, of which $S A D 1$ has a much higher transcriptional level than $S A D 2$ and is considered as the major contributor of $C 18: 1^{\Delta 9}$ formation [18, 37]. In addition to C18:0-ACP, SAD1 accepts C16:0-ACP as the substrate for desaturation, yet in a considerably lower activity [156]. Other C. zofingiensis FADs include FAD2, FAD3,
FAD4, FAD5, FAD6, FAD7 (Fig. 5) [37]. Both FAD2 and FAD6 are $\omega-6$ desaturases: FAD2 is ER-localized and catalyzes desaturation at the $\Delta 12$ position of $C 18: 1^{\Delta 9}$, while FAD6 is chloroplast-localized and likely catalyzes desaturation at the $\Delta 12$ position of $C 18: 1^{\Delta 9}$ and $\Delta 10$ position of $C 16: 1^{\Delta 7}[141,157]$. FAD7, on the other hand, resides in the chloroplast envelop and likely accesses both extrachloroplastic and chloroplastic glycerolipids for the desaturation of $\mathrm{C} 18: 2^{\Delta 9,12}$ and $\mathrm{C} 18: 3^{\Delta 6,9,12}$ at their $\Delta 15$ position and of $C 16: 2^{\Delta 7,10}$ at its $\Delta 13$ position [158]. FAD4 and FAD5 are believed to act on the $\Delta 3$ position (trans) of C16:0 in PG and $\Delta 7$ position of C16:0 in MGDG, respectively [141]. Finally, FAD3 is likely to catalyze desaturation at the $\Delta 4$ position of $C 16$ fatty acyls and $\Delta 6$ position of $\mathrm{C} 18$ fatty acyls [18]. The function of these membranebound FADs from C. zofingiensis, however, is awaiting experimental verification. Considering their transcriptional expression patterns and fatty acid changes upon stress conditions, these FADs may cooperate in a well manner and regulate desaturation degree of fatty acids in C. zofingiensis [18, 37].

Free fatty acids, on the other hand, can enter $\beta$-oxidation pathway for degradation. The location of fatty acid $\beta$-oxidation depends on organisms, e.g., peroxisomes for vascular plants and yeast, both peroxisomes and mitochondria for mammalian cells and probably microalgae [159]. Based on the study in C. reinhardtii [160], fatty acid $\beta$-oxidation in green microalgae is likely to occur in peroxisomes, similar to that in vascular plants [161]. Free fatty acids, once imported into peroxisomes, are converted to acyl-CoAs by peroxisome-localized LACS and then undergo oxidation via a cyclic reaction of four enzymatic steps: oxidation, hydration, dehydrogenation and thiolytic cleavage of an acyl-CoA. These steps involve acyl-CoA oxidase (AOX), enoyl-CoA hydratase (ECH), 3-hydroxyacyl-CoA dehydrogenase (HCD) and 3-ketoacyl-CoA thiolase (KATO) (Fig. 5). In C. zofingiensis, the four enzymes all have peroxisomal forms and their transcriptional expression tends to be down-regulated under several TAG inducing conditions $[18,37]$, suggesting fatty acid $\beta$-oxidation impairment contributes to TAG accumulation. C. zofingiensis has five isoforms of AOX and they may be functionally redundant, as is the case in C. reinhardtii [160]. A summary of genes involved in fatty acid biosynthesis, desaturation and $\beta$-oxidation in C. zofingiensis is listed in Table 2.

\section{Membrane glycerolipid biosynthesis and turnover}

The membrane glycerolipids in C. zofingiensis can be grouped into three categories: glycolipids (MGDG, DGDG and SQDG), phospholipids (PG, PC, PE and PI) and betaine lipid (DGTS) (Fig. 4). In general, the membrane glycerolipid metabolism in green algae is similar 
Table 2 Putative genes involved in fatty acid biosynthesis, desaturation and degradation in C. zofingiensis

\begin{tabular}{|c|c|c|c|c|}
\hline \multirow{2}{*}{$\begin{array}{l}\text { Gene name } \\
\text { abbreviations }\end{array}$} & \multicolumn{2}{|l|}{ Gene ID } & \multirow[t]{2}{*}{ Gene description } & \multirow[t]{2}{*}{ References } \\
\hline & JGI v5.2.3.2 & GenBank & & \\
\hline \multicolumn{5}{|c|}{ Acetyl-CoA production } \\
\hline PDHCE1A & Cz03g08090 & & Pyruvate dehydrogenase complex, E1 a-subunit & \\
\hline PDHC E1B & Cz01g37230 & & Pyruvate dehydrogenase complex, E1 $\beta$-subunit & \\
\hline ACS1 & Cz09g15060 & MK886788 & Acetyl-CoA synthetase & {$[202]$} \\
\hline ACS2 & Cz12g10100 & MK886789 & Acetyl-CoA synthetase & {$[202]$} \\
\hline \multicolumn{5}{|c|}{ De novo fatty acid synthesis } \\
\hline $\mathrm{a}-\mathrm{CT}$ & Cz02g12030 & & Carboxyltransferase a-subunit (ACCase complex) & \\
\hline$\beta-C T$ & Cz02g17060 & & Carboxyltransferase $\beta$-subunit (ACCase complex) & \\
\hline BCCP & Cz03g28270 & & Biotin carboxyl carrier protein (ACCase complex) & \\
\hline BC & Cz13g10110 & GQ996717 & Biotin carboxylase (ACCase complex) & {$[156]$} \\
\hline MCT & Cz13g05150 & & Malonyl-CoA:acyl carrier protein transacylase & \\
\hline KASI & CzO2g14160 & & 3-ketoacyl-ACP synthase, I & \\
\hline KASII & UNPLg00257 & & 3-ketoacyl-ACP synthase, II & \\
\hline KASIIII & Cz18g03070 & & 3-ketoacyl-ACP synthase, III & \\
\hline KAR & Cz01g34370 & & 3-ketoacyl-ACP reductase & \\
\hline HAD & Cz01g09160 & & 3-ketoacyl-ACP dehydratase & \\
\hline ENR & Cz11g20040 & & Enoyl-ACP reductase & \\
\hline \multicolumn{5}{|c|}{ Fatty acid activation and export } \\
\hline FAT & Cz04g05080 & & Acyl-ACP thioesterase & \\
\hline FAX1 & Cz01g44210 & & Fatty acid export & \\
\hline FAX2 & Cz08g09020 & & Fatty acid export & \\
\hline FAX3 & Cz02g41140 & & Fatty acid export & \\
\hline LACS1 & Cz12g27140 & MN317384 & Long-chain acyl-CoA synthetase & [151] \\
\hline LACS2 & Cz11g20120 & MN317385 & Long-chain acyl-CoA synthetase; ER ${ }^{\mathrm{a}}$ & [151] \\
\hline LACS3 & Cz01g36150 & MN317386 & Long-chain acyl-CoA synthetase; ER ${ }^{a}, L^{a}$ & {$[151]$} \\
\hline LACS4 & Cz07g22230 & MN317387 & Long-chain acyl-CoA synthetase; ER ${ }^{\mathrm{a}}$, LD & {$[151]$} \\
\hline LACS6 & Cz08g09130 & MN317389 & Long-chain acyl-CoA synthetase & {$[151]$} \\
\hline \multicolumn{5}{|c|}{ Fatty acid desaturation } \\
\hline SAD1 & Cz04g09090 & GQ996719 & Stearoyl-ACP desaturase & {$[156]$} \\
\hline SAD2 & Cz13g17200 & & Stearoyl-ACP desaturase & \\
\hline FAD6A & Cz08g04110 & & $\omega-6$ fatty acid desaturase, chloroplastic type & \\
\hline FAD6B & Cz11g21120 & & $\omega-6$ fatty acid desaturase like, chloroplastic type & \\
\hline FAD2 & Cz03g33220 & & $\omega-6$ fatty acid desaturase, ER type & \\
\hline FAD7A & Cz04g31180 & & $\omega-3$ fatty acid desaturase & \\
\hline FAD7B & Cz06g28130 & & $\omega-3$ fatty acid desaturase & \\
\hline FAD5A & Cz07g00120 & & MGDG-specific palmitate $\Delta 7$ desaturase & \\
\hline FAD5B & Cz06g00170 & & MGDG-specific palmitate $\Delta 7$ desaturase & \\
\hline FAD5C & Cz13g01140 & & MGDG-specific palmitate $\Delta 7$ desaturase like & \\
\hline FAD3A & CzO6g12050 & & $\Delta 4 / \Delta 6$ desaturase like & \\
\hline FAD3B & UNPLg00012 & & $\Delta 4 / \Delta 6$ desaturase like & \\
\hline FAD4 & Cz12g10230 & & $\triangle 3$ palmitate desaturase & \\
\hline \multicolumn{5}{|c|}{ Fatty acid $\beta$-oxidation } \\
\hline LACS5 & Cz05g30060 & MN317388 & Long-chain acyl-CoA synthetase, peroxisome ${ }^{\mathrm{a}}$ & {$[151]$} \\
\hline $\mathrm{AOX} 1$ & Cz16g14110 & & Acyl-CoA oxidase & \\
\hline $\mathrm{AOX} 2$ & Cz07g30210 & & Acyl-CoA oxidase & \\
\hline AOX3 & Cz07g18040 & & Acyl-CoA oxidase & \\
\hline AOX4 & Cz08g04130 & & Acyl-CoA oxidase & \\
\hline AOX5 & Cz17g14150 & & Acyl-CoA oxidase & \\
\hline
\end{tabular}


Table 2 (continued)

\begin{tabular}{llll}
\hline $\begin{array}{l}\text { Gene name } \\
\text { abbreviations }\end{array}$ & Gene ID & & Gene description \\
\cline { 2 - 3 } & JGl v5.2.3.2 & GenBank & \\
\hline ECH1 & Cz16g07140 & enoyl-CoA hydratase \\
ECH2 & Cz03g36260 & enoyl-CoA hydratase \\
ECH3 & CzO4g19010 & Enoyl-CoA hydratase/isomerase \\
ECH4 & CzO6g10230 & Enoyl-CoA hydratase \\
ECH5 & Cz09g10030 & Enoyl-CoA hydratase \\
HCD & Cz11g22170 & 3-hydroxyacyl-CoA dehydrogenase \\
KATO & Cz06g36270 & 3-ketoacyl-CoA thiolase \\
\hline
\end{tabular}

${ }^{a}$ Where experimental evidence of a subcellular localization is available

to that in vascular plants, except that green algae often contain DGTS and thus its metabolic pathway, while vascular plants lack it (Fig. 5) [162]. MGDG and DGDG, the major chloroplastic lipid fractions, are synthesized in the chloroplast. Using diacylglycerol (DAG) as the acceptor, the galactose moiety from UDP-galactose is transferred leading to MGDG formation, which is catalyzed by MGDG synthase (MGD). An additional transfer of the galactose moiety from UDP-galactose to MGDG, mediated by DGDG synthase (DGD), results in the formation of DGDG. SQDG, another chloroplastic lipid class that plays an important role in photosynthesis, is also biosynthesized in the chloroplast, which involves UDP-sulfoquinovose synthase (SQD1) and SQDG synthase (SQD2) that catalyze UDP-sulfoquinovose formation and transfer of sulfoquinovose from UDP-sulfoquinovose to DAG for SQDG synthesis, respectively [163]. Compared to $C$. reinhardtii that has only one gene copy for each $M G D$, DGD, SQD1 and SQD2 [164], C. zofingiensis harbors one copy for $M D G, S Q D 1$ and $S Q D 2$ each yet three copies for $D G D$ [37]. Upon exposure of $C$. zofingiensis to stress conditions, MGDG, DGDG and SQDG all decreased, yet their biosynthetic pathways showed no transcriptional down-regulation [13, 17, 18, 37, 39].

Of the phospholipids, PG is believed to reside predominantly in the chloroplast and plays a role in photosystem II [165]. In addition, when subjected to sulfur deficient conditions, PG may accumulate and compensate for SQDG impairment to maintain photosystem I activity [166]. Unlike other chloroplastic membrane lipids, PG biosynthesis starts from cytidine diphosphate DAG (CDP-DAG), a product from the condensation of phosphatidic acid (PA) and cytidine triphosphate mediated by phosphatidate cytidylyltransferase (CDS). Through the action of phosphatidylglycerophosphate synthase (PGPS) on CDP-DAG and glycerol-3-phosphate (G3P), phosphatidylglycerophosphate is formed, which is further converted to PG by phosphatidylglycerophosphatase
(PGP). C. zofingiensis is predicted to contain two CDS genes, one PGPS gene and one PGP gene [37]. Similarly, the transcriptional expression pattern of these genes is inconsistent with PG decrease observed under stress conditions [18, 37, 39]. PI also uses CDP-DAG as the precursor for synthesis, catalyzed by phosphatidylinositol synthase (PIS). There are two PIS-encoding genes present in C. zofingiensis [37]. Although C. zofingiensis harbors a gene encoding CDP-DAG-dependent phosphatidylserine (PS) synthase (PSS), no detectable level of PS is observed. This is probably due to that PS is rapidly converted to PE by PS decarboxylase (PSD), which is present in C. zofingiensis [37]. PE can also be synthesized from the CDP-ethanolamine pathway in which ethanolamine kinase (ETK), CDP-ethanolamine synthase (ECT) and ethanolaminephosphotransferase (EPT) are involved. $\mathrm{PC}$, on the other hand, can be synthesized from the CDP-choline pathway and/or the methylation of PE; the former involves choline kinase (CHK), CDP-choline synthase (CCT) and cholinephosphotransferase (CPT) [167]. Similar to Cyanidioschyzon merolae and several Chlamydomonas species [168], C. zofingiensis possesses a single bifunctional EPT/CPT enzyme that is believed to catalyze the last biosynthetic step of both PE and PC [37]. As for DGTS, it is synthesized from DAG and $S$-adenosylmethionine by the action of DGTS synthase (BTA) [164]. Similar in C. reinhardtii, a single BTA gene is present in C. zofingiensis. Table 3 summarizes the putative genes involved in membrane glycerolipid biosynthesis in C. zofingiensis.

Considering that the decreases of membrane glycerolipids upon stress conditions are accompanied with no transcriptional down-regulation of their biosynthetic pathways [18, 37, 39], we hypothesize that their biosyntheses are maintained yet catabolic pathways mediated by lipases are likely stimulated leading to net decreases of these lipids. Microalgae harbor a number of genes encoding putative lipases, yet Plastid Galactoglycerolipid 
Table 3 Putative genes involved in membrane glycerolipid biosynthesis and turnover in C. zofingiensis

\begin{tabular}{|c|c|c|c|c|}
\hline \multirow{2}{*}{$\begin{array}{l}\text { Gene name } \\
\text { abbreviations }\end{array}$} & \multicolumn{2}{|l|}{ Gene ID } & \multirow[t]{2}{*}{ Gene description } & \multirow[t]{2}{*}{ References } \\
\hline & JGI v5.2.3.2 & GenBank & & \\
\hline \multicolumn{5}{|c|}{ Glycolipid synthesis (MGDG, DGDG and SQDG) } \\
\hline GALE1 & Cz12g16020 & & UDP-galactose 4-epimerase & \\
\hline GALE2 & Cz08g10110 & & UDP-galactose 4-epimerase & \\
\hline MGD & Cz08g30040 & & Monogalactosyldiacylglycerol synthase & \\
\hline DGD1 & Cz03g26070 & & Digalactosyldiacylglycerol synthase & \\
\hline DGD2 & Cz10g17090 & & Digalactosyldiacylglycerol synthase & \\
\hline DGD3 & Cz13g19030 & & Digalactosyldiacylglycerol synthase & \\
\hline UGPase & UNPLg00641 & & UDP-glucose pyrophosphorylase & \\
\hline SQD1 & Cz03g31030 & & UDP-sulfoquinovose synthase & \\
\hline SQD2 & Cz07g23140 & & Sulfoquinovosyldiacylglycerol synthase & \\
\hline \multicolumn{5}{|c|}{$P G$ and $P I$ synthesis } \\
\hline CDS1 & Cz10g20080 & & Phosphatidate cytidylyltransferase & \\
\hline CDS2 & Cz01g36190 & & Phosphatidate cytidylyltransferase & \\
\hline PGPS & Cz01g26070 & & Phosphatidylglycerophosphate synthase & \\
\hline PGP & Cz12g07050 & & Phosphatidylglycerophosphatase & \\
\hline MIPS & Cz01g18130 & & myo-inositol-1-phosphate synthase & \\
\hline PIS1 & Cz01g00060 & & phosphatidylinositol synthase & \\
\hline PIS2 & Cz17g13240 & & phosphatidylinositol synthase & \\
\hline \multicolumn{5}{|c|}{ DGTS, PC and PE synthesis } \\
\hline SAS1 & Cz15g18200 & & S-adenosylmethionine synthase & \\
\hline SAS2 & Cz05g24030 & & S-adenosylmethionine synthase & \\
\hline BTA & Cz01g13260 & & Betaine lipid synthase & \\
\hline $\mathrm{CHK}$ & UNPLg00491 & & Choline kinase & \\
\hline $\mathrm{CCT}$ & Cz12g21150 & & Choline-phosphate cytidylyltransferase & \\
\hline EPT/CPT & Cz05g09130 & & Ethanolaminephosphotransferase/cholinephosphotransferase & \\
\hline ETK & Cz11g15030 & & Ethanolamine kinase & \\
\hline $\mathrm{ECT}$ & Cz05g17180 & & CDP-Ethanolamine synthase & \\
\hline \multicolumn{5}{|c|}{ Putative membrane lipid lipases } \\
\hline PGD1 & Cz01g38020 & & Plastid Galactoglycerolipid Degradation1, Lipase class 3 & \\
\hline- & Cz02g15090 & & Alpha/beta hydrolase family & \\
\hline- & Cz03g14190 & & Alpha/beta hydrolase family & \\
\hline- & Cz01g06170 & & Lipase, SF153 & {$[40]$} \\
\hline- & Cz12g10010 & & Lipase, SF153 & {$[40]$} \\
\hline
\end{tabular}

Degradation1 (PGD1) from C. reinhardtii is the only one that has been demonstrated to be involved in membrane lipid turnover [169]. This lipase, required for normal structure of thylakoid membranes, acts specifically on the $s n-1$ position of MGDG to release C18:1 ${ }^{\Delta 9}$ mainly for supporting TAG synthesis and is important during acclimation of $C$. reinhardtii to various adverse conditions $[169,170]$. A single $P G D 1$ gene is present in the genome of C. zofingiensis, which shows a considerable up-regulation at the transcriptional level under multiple stress conditions, well consistent with the severe degradation of MGDG [18, 32, 37-39]. If C. zofingiensis PGD1 has the same function as its homolog in C. reinhardtii, which of course needs experimental verification, additional lipases are required to support the degradation of other chloroplastic lipids, such as DGDG, SQDG and PG. It has been suggested that Cz02g15090 and Cz03g14190 may encode such lipases as they cluster with $P G D 1$ based on the transcriptional expression pattern and are highly up-regulated under ND conditions [37]. Moreover, proteomics analysis of the $\mathrm{LD}$ fraction from C. zofingiensis has identified two lipases (Cz01g06170 and Cz12g10010), which are transcriptionally up-regulated upon ND and can enable yeast cells to produce more TAG when heterologously expressed, indicating that the two lipases may act on membrane lipids (of LDs and/or membrane contact 
sites between LDs and ER and between LDs and chloroplast) that they can access and contribute fatty acids to TAG synthesis [40]. Nevertheless, under SD and SS conditions that also cause severe degradation of chloroplastic lipids, the above mentioned four lipase genes exhibit no transcriptional up-regulation [18, 39]. Whether they are bona fide membrane lipid lipases and what lipid substrates they prefer are awaiting experimental evidences.

Interestingly, it has been reported that phospholipid:diacylglycerol acyltransferase (PDAT) from C. reinhardtii, in addition to functioning as an acyltransferase involved in TAG biosynthesis, has lipase activity toward a broad range of glycolipids and phospholipids, as suggested by the in vitro enzymatic assays [171]. Seemingly, PDAT in microalgae, transcriptionally up-regulated by ND, contributes to membrane lipid turnover in microalgae $[171,172]$, similar to the role of its homolog in vascular plants [173]. The gene encoding PDAT in $C$. zofingiensis is also up-regulated by ND as well as other stress conditions, yet the up-regulation extent is only moderate [18, 32, 37, 39], indicative of its mild contribution to membrane lipid turnover.

\section{TAG biosynthesis and degradation}

In general, as in vascular plants, TAG biosynthesis in microalgae is believed to perform through two pathways, the acyl-CoA-dependent Kennedy pathway and the acyl-CoA-independent pathway [162]. The former pathway involves a series of enzymatic reactions catalyzed in succession by glycerol-3-phosphate acyltransferase (GPAT), 1-acyl-sn-glycerol-3-phosphate acyltransferase (LPAAT), phosphatidate phosphatase (PAP) and diacylglycerol acyltransferase (DGAT). GPAT mediates the first step of the acyl-CoA-dependent pathway leading to lysophosphatidic acid (LPA) formation by transferring the acyl moiety from an acylCoA to the $s n-1$ or $s n-2$ position of G3P [174]. Differing from vascular plants that harbor a high dose of GPAT isoforms [174], microalgae generally contain one chloroplastic form and one extrachloroplastic ER-localized form, which has been indicated in the green algae $C$. reinhardtii [141], Monoraphidium neglectum [175], C. zofingiensis [37] and Lobosphaera incisa [176], the heterokont algae Nannochloropsis oceanica [177] and P. tricornutum [178], and the red alga Cyanidioschyzon merolae [179]. In C. zofingiensis, the extrachloroplastic GPAT2 rather than the chloroplastic GPAT1 shows transcriptional up-regulation under multiple TAG inducing conditions and contributes to ND-associated TAG biosynthesis $[18,37,39]$. Similarly, it is believed that the extrachloroplastic GPAT (ER-localized) from L. incisa and C. merolae is involved in TAG biosynthesis $[176,179]$. By contrast, in the diatom P. tricornutum, the chloroplastic GPAT seemingly plays a role in TAG synthesis, as suggested by its overexpression results [180]. The substrate preference of GPAT determines the fatty acid composition of $s n-1$ position of TAG. Considering that $C$. zofingiensis TAG sn-1/3 consists mainly of $\mathrm{C} 18: 1^{\Delta 9}$ [17], GPAT2 may prefer $\mathrm{C} 18: 1^{\Delta 9}$-CoA as the acyl donor.

LPAAT catalyzes the second acylation step by transferring the acyl moiety from an acyl-CoA to $s n-2$ position of LPA leading to PA formation. LPAAT also has both chloroplastic and extrachloroplastic forms in algae and the number varies depending on algal species $[37,141$, $175,177,178]$. It has been reported that the chloroplastic LPAAT of $C$. reinhardtii (CrLPAAT1), up-regulated by ND, prefers $16: 0-\mathrm{CoA}$ over $\mathrm{C} 18: 1^{\Delta 9}$-CoA as the acyl donor for PA synthesis and is involved in TAG synthesis [181]. Consistent with the acyl-CoA preference of CrLPAAT1, overexpression of CrLPAAT1 in C. reinhardtii promotes increase of TAG with $s n-2$ position being C16 acyls [181]. Interestingly, CrLPAAT2, an ER-localized chlorophyte-specific LPAAT enzyme, also prefers 16:0CoA over C18: $1^{\Delta 9}$-CoA for PA formation, distinguishing from the canonical ER form of LPAAT that generally utilizes C18-CoAs as the acyl donor [182]. This is reasonable as $s n-2$ position of TAG in C. reinhardtii consists predominantly of C16:0 [183, 184]. By contrast, $C$. zofingiensis synthesizes TAG with $s n-2$ position mainly being $C 18: 1^{\Delta 9}[17]$. These may reflect the great difference in acyl-CoA preference of LPAATs between the two closely related green algae $C$. reinhardtii and $C$. zofingiensis. There are three LPAAT isoforms in C. zofingiensis: LPAAT1 (homolog to CrLPAAT1), LPAAT2 (homolog to CrLPAAT2), and LPAAT3 [37]. As is the case in C. reinhardtii, both C. zofingiensis LPAAT1 and LPAAT2 genes are considerably up-regulated by ND, indicative of their involvement in TAG synthesis [37]. Whether the two LPAATs have substrate preference on C18-CoAs and to what extent they contribute to TAG synthesis are awaiting clarification via such experiments as in vitro enzymatic assays and in vivo functional characterization.

Prior to utilization for TAG synthesis, PA needs to be converted to DAG by the action of PAP. There is only one report about functional dissection of algal PAP, in which an extrachloroplastic PAP from C. reinhardtii, upregulated transcriptionally by $\mathrm{ND}$, contributes to TAG synthesis as suggested by both overexpression and suppression experiments [185]. C. zofingiensis harbors three putative PAP isoforms, one chloroplastic form (PAP1) and two extrachloroplastic forms (PAP2 and PAP3) [37]. Interestingly, these $P A P$ genes respond differentially upon various stress conditions of ND, SD and SS: PAP1 is up-regulated by ND, PAP3 is up-regulated by $\mathrm{SD}$ and SS, while PAP3 shows no up-regulation $[18,37,39]$. This 
indicates that C. zofingiensis may adopt different PAPs to cope with different stresses for TAG synthesis.

DGAT catalyzes the last and committed step in the Kennedy pathway for TAG synthesis by transferring the acyl moiety from an acyl-CoA to the $s n-3$ position of a DAG. There are three DGAT types, the membranebound type I (DGAT1) and type II (DGAT2 or DGTT) and the soluble type III (DGAT3) [186]. In general, microalgae harbor a much larger number of DGAT isoforms than vascular plants (e.g., one versus eleven for the type II), pointing to more complex regulations of microalgal TAG synthesis. Although why microalgae need such high dose of DGATs remains less understood, functional characterization of DGATs from multiple aspects has been conducted for many species including $C$. reinhardtii [183, 187, 188], C. zofingiensis [189-191], H. pluvialis [192, 193], N. oceanica [194-196], and P. tricornutum [197-199]. C. zofingiensis harbors ten putative DGAT isoforms, two type I (DGAT1A and DGAT1B) and eight type II (DGTT1 through DGTT8); all are predicted to be extrachloroplast-targeted [189]. For the transcriptional expression pattern upon ND, DGAT1A, DGTT1, DGTT5, DGTT6 and DGTT8 are considerably up-regulated, while the left five show slight or little variation $[37,189]$. It is worth noting that not all ten DGAT isoforms have observed activity to restore TAG synthesis in a TAGdeficient yeast mutant $[189,190]$. It seems not surprising as this phenomenon happens for other algae when expressing their DGAT genes in the same yeast mutant [183, 192-195, 199-201]. The functional failure of some putative algal DGATs in yeast may be attributed to (1) they are not bona fide DGAT enzymes, (2) their protein expression levels are too low to function or the expressed proteins are misassembled into nonfunctional forms in yeast, and (3) certain substrates or co-factors essential for the DGAT activity are absent from yeast, etc. Of the seven functional DGATs from C. zofingiensis based on the functional complementation results, DGAT1A has the highest activity followed by DGTT5, which is also supported by the in vitro DGAT assays using a wide range of substrates [189]. Clearly, DGAT1A and DGTT5, both residing at ER, have overlapping yet distinctive substrate specificity for both acyl-CoAs and DAGs: DGAT1A prefers eukaryotic DAGs with strong activity on C16:0- and C18: $1^{\Delta 9}$-CoAs, while DGTT5 prefers prokaryotic DAGs with weak activity on C16:0- and C18:1 ${ }^{\Delta 9}$-CoAs. Taken into account the transcriptional expression levels, functional complementation results in yeast, in vitro DGAT assays and the fatty acid composition in $s n-2$ and $s n-1 / 3$ positions of TAG [17, 189], DGAT1A likely contributes more than DGTT5 to ND-induced TAG in C. zofingiensis. Unlike ND, SD and SS stimulate the transcriptional expression of DGTT5 but not DGAT1A $[18,39]$. This may partly explain why $C$. zofingiensis has a considerably higher TAG level under ND conditions as compared to under SD and SS conditions $[17,202]$ and further support the important role of DGAT1A in TAG synthesis. Interestingly, DGAT1A and DGTT5 possess strong activity on the CoA forms of $\omega-3$ polyunsaturated fatty acids, such as eicosapentaenoyl-CoA (EPA-CoA) and docosahexaenoylCoA (DHA-CoA) [189]. In this context, DGAT1A and $D G T T 5$ have the potential to serve as promising gene targets of engineering for not only enhancing TAG production but also enriching $\omega-3$ polyunsaturated fatty acids in TAG to add nutritional benefits.

The acyl-CoA-independent pathway for TAG synthesis is mediated by PDAT, which, differing from DGAT that uses acyl-CoAs, transfers the acyl from lipids (mainly the $s n-2$ position of phospholipids) to the $s n-3$ position of a DAG [203]. The enzyme has been named as PDAT, because the phospholipid PC was used as the acyl donor for investigating in vitro enzymatic activities in the pioneering study [204]. In fact, PDAT can utilize not only phospholipids but many other substrates as acyl donors, yet the activity and substrate preference are dependent on the PDAT sources [171, 204, 205]. Seemingly, PDAT functions more under non-stress than under stress conditions and its contribution to TAG synthesis is minor as compared to DGATs in C. reinhardtii $[71,171,183]$. In C. zofingiensis, $P D A T$ is up-regulated under various TAG inducing conditions, yet in a less extent than DGAT1A and DGTT5 [18, 37, 39], suggesting its minor contribution to TAG synthesis, as is the case in C. reinhardtii.

TAG accumulation is dependent on not only biosynthesis but also catabolism. Sugar-Dependent1 (SDP1) represents one of the most well studied TAG lipases, which was first characterized in Arabiodopsis [206]. This TAG lipase, similar to the yeast triacylglycerol lipase 3 and human adipose triglyceride lipase that harbor a patatin-like acyl-hydrolase domain, is LDassociated and acts mainly on TAG for releasing free fatty acids [206]. SDP1 homologs and their roles in TAG degradation have been reported in several microalgae including P. tricornutum [207], L. incise [208], N. oceanica [209] and C. reinhardtii [210]. C. zofingiensis contains a single SPD1-encoding gene, which is transcriptionally down-regulated under several TAGinducing conditions [18, 37-39], suggesting the role of SDP1 in TAG breakdown in this alga as well. Moreover, in C. zofingiensis, another lipase (Cz02g29090) has a more severe down-regulation at its transcriptional level than SDP1 under stress conditions that induce TAG accumulation [18, 37, 39]. This lipase, homologous to AtLip1 from Arabidopsis with confirmed TAG lipase activity [211], is up-regulated upon removal of the stress that leads to TAG degradation [39]. In this 
context, Cz02g29090 may encode a TAG lipase and play a more important role than SDP1 in TAG catabolism in C. zofingiensis. Functional characterization of these lipases will help understand oleaginousness of this alga. The putative genes involved in TAG biosynthesis and catabolism in C. zofingiensis are listed in Table 4.

\section{Roles of LDs in TAG metabolism}

As is the case in vascular plants, TAG, once synthesized, is packed into LDs for storage in algae [212]. LD is an organelle composed of an outer monolayer of polar lipids and a hydrophobic core filled with TAG and/or sterols; the outer monolayer is equipped with many proteins, such as structural proteins that maintain LD and functional enzymes [213]. In addition to serving as a reservoir

Table 4 Putative genes involved in TAG biosynthesis and degradation in C. zofingiensis

\begin{tabular}{|c|c|c|c|c|}
\hline \multirow{2}{*}{$\begin{array}{l}\text { Gene name } \\
\text { abbreviations }\end{array}$} & \multicolumn{2}{|l|}{ Gene ID } & \multirow[t]{2}{*}{ Gene description } & \multirow[t]{2}{*}{ References } \\
\hline & JGI v5.2.3.2 & GenBank & & \\
\hline \multicolumn{5}{|l|}{ G3P production } \\
\hline GPDH1 & Cz12g24180 & & Glycerol-3-phosphate dehydrogenase & \\
\hline GPDH2 & Cz04g17090 & & Glycerol-3-phosphate dehydrogenase & \\
\hline GPDH3 & Cz10g29180 & & Glycerol-3-phosphate dehydrogenase & \\
\hline GPDH4 & Cz08g08240 & & Glycerol-3-phosphate dehydrogenase, mitochondrial form & \\
\hline GK1 & Cz12g27090 & & Glycerol kinase & \\
\hline GK2 & Cz18g13070 & & Glycerol kinase & \\
\hline GK3 & Cz05g35210 & & Glycerol kinase & \\
\hline \multicolumn{5}{|l|}{ TAG biosynthesis } \\
\hline GPAT1 & Cz11g03260 & & Glycerol-3-phosphate acyltransferase, chloroplastic & [37] \\
\hline GPAT2 & Cz09g31330 & & Glycerol-3-phosphate acyltransferase & [37] \\
\hline LPAAT1 & Cz16g02090 & & 1-acyl-sn-glycerol-3-phosphate acyltransferase, chloroplastic & \\
\hline LPAAT2 & Cz04g14150 & & 1-acyl-sn-glycerol-3-phosphate acyltransferase & \\
\hline LPAAT3 & Cz10g20070 & & 1-acyl-sn-glycerol-3-phosphate acyltransferase & \\
\hline PAP1 & Cz05g23060 & & Phosphatidate phosphatase, Lipin & \\
\hline PAP2 & Cz10g16040 & & Phosphatidate phosphatase & \\
\hline PAP3 & Cz16g11240 & & Phosphatidate phosphatase & \\
\hline DGAT1A & Cz06gg05010 & MH523419 & Diacylglycerol acyltransferase, type I; ER ${ }^{a}$ & [189] \\
\hline DGAT1B & Cz09g08290 & MH523420 & Diacylglycerol acyltransferase, type I & [189] \\
\hline DGTT1 & Cz06g35060 & MH523421 & Diacylglycerol acyltransferase, type II & [189] \\
\hline DGTT2 & Cz06g22030 & MH523422 & Diacylglycerol acyltransferase, type II & [189] \\
\hline DGTT3 & Cz09g23010 & MH523423 & Diacylglycerol acyltransferase, type II & [189] \\
\hline DGTT4 & Cz11g24150 & MH523424 & Diacylglycerol acyltransferase, type ॥ & [189] \\
\hline DGTT5 & Cz09g27290 & MH523425 & Diacylglycerol acyltransferase, type II; ER & [189] \\
\hline DGTT6 & Cz15g22140 & MH523426 & Diacylglycerol acyltransferase, type II & [189] \\
\hline DGTT7 & Cz11g21100 & MH523427 & Diacylglycerol acyltransferase, type II & [189] \\
\hline DGTT8 & Cz08g14220 & MH523428 & Diacylglycerol acyltransferase, type ॥ & [189] \\
\hline PDAT & Cz10g07180 & & Phospholipid:diacylglycerol acyltransferase & \\
\hline \multicolumn{5}{|c|}{ LD structural proteins } \\
\hline MLDP & Cz04g29220 & & Major lipid droplet protein; LD & [40] \\
\hline CLS1 & Cz16g16140 & & Caleosin related protein; LD & [40] \\
\hline CLS2 & Cz09g31050 & & Caleosin related protein; LDa & [40] \\
\hline CLS3 & Cz09g11210 & & Caleosin related protein; $L^{a}$ & [40] \\
\hline CLS4 & Cz03g13150 & & Caleosin related protein; LDa & {$[40]$} \\
\hline \multicolumn{5}{|c|}{ Putative lipases involved in TAG degradation } \\
\hline SDP1 & Cz05g29160 & & Sugar-Dependent1 TAG lipase & \\
\hline- & Cz02g29090 & & Putative TAG lipase & \\
\hline
\end{tabular}

${ }^{\text {a }}$ Where experimental evidence of a subcellular localization is available 
for neutral lipids, LD is believed to play roles in many biological processes, such as lipid homeostasis, signaling, membrane trafficking, etc. [213-215]. Proteomic studies of LD fraction, which help understand LD biology and lipid metabolism, have been conducted for many algae including C. reinhardtii [216-218], N. oceanica [219], Fistulifera sp. [220], Dunaliella bardawil [221], L. incise [208], P. tricornutum [222], C. zofingiensis [40], and Parachlorella kessleri [223].

In C. zofingiensis, the LD fraction consists predominantly of TAG (over 90\%), with a very low level of polar lipids [40]. The LD proteins can be classified mainly into functional unknown group, lipid metabolism, carbon metabolism and vesicle trafficking. Similar to in the other green algae, the most abundant LD protein in $C$. zofingiensis is the Major Lipid Droplet Protein (MLDP) [40], which is drastically up-regulated by stress conditions and correlates well with TAG accumulation [32, 37, 39]. MLDP, differing from oleosin, the major LD protein of vascular plants that possesses a long hydrophobic segment stretching into the TAG matrix of LDs [213], has no hydrophobic segment and resides on the surface of LD in a relatively loose association probably due to its intrinsic hydrophobic and topological properties [224, 225]. Expression of C. zofingiensis MLDP can restore the phenotypes (LD size and number and TAG content) of a C. reinhardtii mutant with insertional disruption in its $M L D P$ gene and promote TAG content in a wild type $C$. reinhardtii strain [40], indicating that MLDP functions in not only maintaining LD but also facilitating TAG accumulation. Probably, MLDP overexpression facilitates sequestration of neutral lipids into LDs for storage, thus attenuating the end-product inhibition on TAG biosynthesis-related enzymes for improved TAG synthesis.

Intriguingly, many $C$. zofingiensis $\mathrm{LD}$ proteins have no homologs present in the LD proteome of $C$. reinhardtii, including certain functional unknown proteins, caleosins and lipases, suggesting the unique characteristic of C. $z$ ofingiensis LDs [40]. Caleosin harbors a central hydrophobic segment and thus can stretch into the mono-layer of LDs for anchoring [226]. Although widely present in LDs of vascular plants, caleosin represents a minor integral LD protein group and has an extremely lower abundance than oleosin [213]. By contrast, in C. zofingiensis LDs, caleosin proeins have comparable abundance to MLDP [40]. Unlike $M L D P$ that is up-regulated at early stages of $\mathrm{ND}$, caleosin genes are only up-regulated at late stages of ND. It is hypothesized that MLDP and caleosins have differential functions in LD biogenesis in C. zofingiensis: while MLDP is involved in formation and maintaining size of nascent LDs, caleosins probably function in fusing nascent LDs to large ones [40]. Moreover, a novel model has been proposed for C. zofingiensis LDs, which have connections with both the ER and chloroplast and are equipped with many structural proteins and functional enzymes: the structural proteins, such as MLDP, caleosins, and certain unknown proteins, are highly abundant and maintain the stability of LDs; by contrast, enzymes, such as polar lipid lipases and LACSs, collaborate with those ER and/or chloroplast-localized ones involved in lipid metabolism (e.g., GPAT, LPAAT, DGAT) to contribute to TAG biosynthesis [40]. It is worth noting that this study only performs a single time point proteomics analysis of LDs under ND conditions. The temporal dynamics of the LD proteome upon ND and differences in LD proteomes among various stress conditions, such as ND, $\mathrm{SD}, \mathrm{SS}$ and $\mathrm{HL}$, are interesting and remain to be further investigated.

\section{Mechanistic insights into lipid metabolism for TAG biosynthesis in C. zofingiensis}

C. zofingiensis has the capacity to synthesize and accumulate high levels of TAG under various stress conditions, yet ND is the most efficient stimulus for triggering TAG accumulation $[13,17,20,32]$. To understand the mechanisms of oleaginousness in C. zofingiensis, a multiomics study has been conducted, which involves a systematical and integrated analysis of time-resolved transcriptomes, lipidomes and metabolomes in response to ND [37]. The massive TAG accumulation in C. zofingiensis upon ND is attributed to coordinated regulation of multiple biological processes, including 1) stimulation of protein and amino acid catabolism, starch catabolism and glycolysis that allocate carbon flux to lipids, acetyl-CoA production via the PDHC and PDHC bypass pathways (providing precursor for de novo fatty acid synthesis), de novo fatty acid synthesis, fatty acid activation and desaturation and membrane lipid turnover (providing acyl-CoAs for TAG assembly), G3P production via the glycerol-3-phosphate dehydrogenase (GPHD)- and glycerol kinase (GK)-mediated pathways, acyltransferases (GPAT, LPAAT and DGAT) for TAG assembly, LD proteins, such as MLDP and caleosins, for LD formation and storage of TAG, ATP production via glycolysis and TCA cycle (providing energy molecules), NADPH production via the oxidative pentose phosphate (OPP) pathway and $\mathrm{NADP}^{+}$-dependent malic enzyme (ME) (providing reductants), and 2) suppression of TAG breakdown and fatty acid $\beta$-oxidation.

Compared to the green algae C. reinhardtii [227] and $M$. neglectum [175] with time-resolved transcriptomes under ND conditions, C. zofingiensis shows several key distinctions regarding oleaginousness for TAG accumulation [37]. First, unlike in C. reinhardtii or M. neglectum the PDHC bypass route contributes more than the chloroplastic PDHC route to acety-CoA production, the 
chloroplastic PDHC route serves as a major source of acety-CoA in C. zofingiensis. Second, regarding the genes involved in de novo fatty acid synthesis in response to ND, most show a well-coordinated up-regulation in $C$. zofingiensis; by contrast, many genes are down-regulated to different degrees in C. reinhardtii and M. neglectum. Third, in C. zofingiensis the ER-localized GPAT rather than the chloroplastic one contributes to ND-induced TAG synthesis, while in $C$. reinhardtii the chloroplastic GPAT likely contributes more than the ER one to TAG synthesis. Fourth, C. zofingiensis is superior to C. reinhardtii in the dose of DGAT isoforms and the abundance of their transcripts thus accumulates a considerably higher level of TAG. Fifth, while consisting of predominantly $\mathrm{C} 16$ fatty acyls in $C$. reinhardtii, the $s n-2$ position of TAG in C. zofingiensis is composed of mainly C18 fatty acyls, suggesting that $C$. zofingiensis, differing from $C$. reinhardtii, employs the eukaryotic pathway rather than the prokaryotic pathway as the major for TAG biosynthesis. Six, C. reinhardtii synthesizes a basal level of starch under favorable growth conditions and shows a transient increase of starch upon ND; by contrast, C. zofingiensis synthesizes starch constantly and the starch level decreases upon ND via stimulating starch degradation, providing carbon precursors for TAG synthesis.

C. zofingiensis accumulates TAG as the carbon and energy reservoir under stress conditions and when the carbon source is in excess, and obviously there are common attributes as well as distinctions in TAG metabolism among these different conditions [18, 33, 37-39, 228]. Nevertheless, how algal cells sense these conditions to trigger TAG synthesis and accumulation remains largely unknown and is worth of deep investigation.

\section{Carotenogenesis for astaxanthin biosynthesis in C. zofingiensis}

The carotenoid profile in C. zofingiensis has been reported by many independent research groups and varies likely due to the use of different culture conditions and analytic methods [22, 24, 32, 33, 41, 54, 55, 104, 107]. In general, C. zofingiensis contains predominantly primary carotenoids including lutein, $\beta$-carotene, zeaxanthin, neoxanthin, violaxanthin, and $\alpha$-carotene under favorable growth conditions, with lutein and $\beta$-carotene being the major ones; upon stress conditions, such as $\mathrm{ND}$, secondary carotenoids including astaxanthin, canthaxanthin, keto-lutein, echinenone, and adonixanthin accumulate and become the dominated portion of carotenoids (Fig. 6). Nevertheless, the astaxathin content in C. zofingiensis, ranging from 0.1 to $1 \%$ of dry weight depending on culture conditions (Table 1 ), is much lower than that in $H$. pluvialis ( $4 \%$ of dry weight). This necessitates the requirements of complicated downstream purification processes for C. zofingiensis astaxanthin, leading to input of more production costs and thus the impairment of commercial potential. Genetic engineering of $C$. zofingiensis may have the potential to break the inherent constraints on astaxanthin accumulation, which relies on a better understanding of carotenogenesis for astaxanthin biosynthesis in this alga. With the assistance of whole genome sequence and reconstruction of carotenogenic pathways [33, 41], carotenogenic genes for synthesis of the carotenoid precursors isopentenyl pyrophosphate (IPP) and dimethylallyl pyrophosphate (DMAPP), of primary carotenoids from IPP/DMAPP, and of astaxanthin from $\beta$-carotene have been identified (Fig. 7 and Table 5), which are detailed in the subsequent sections.

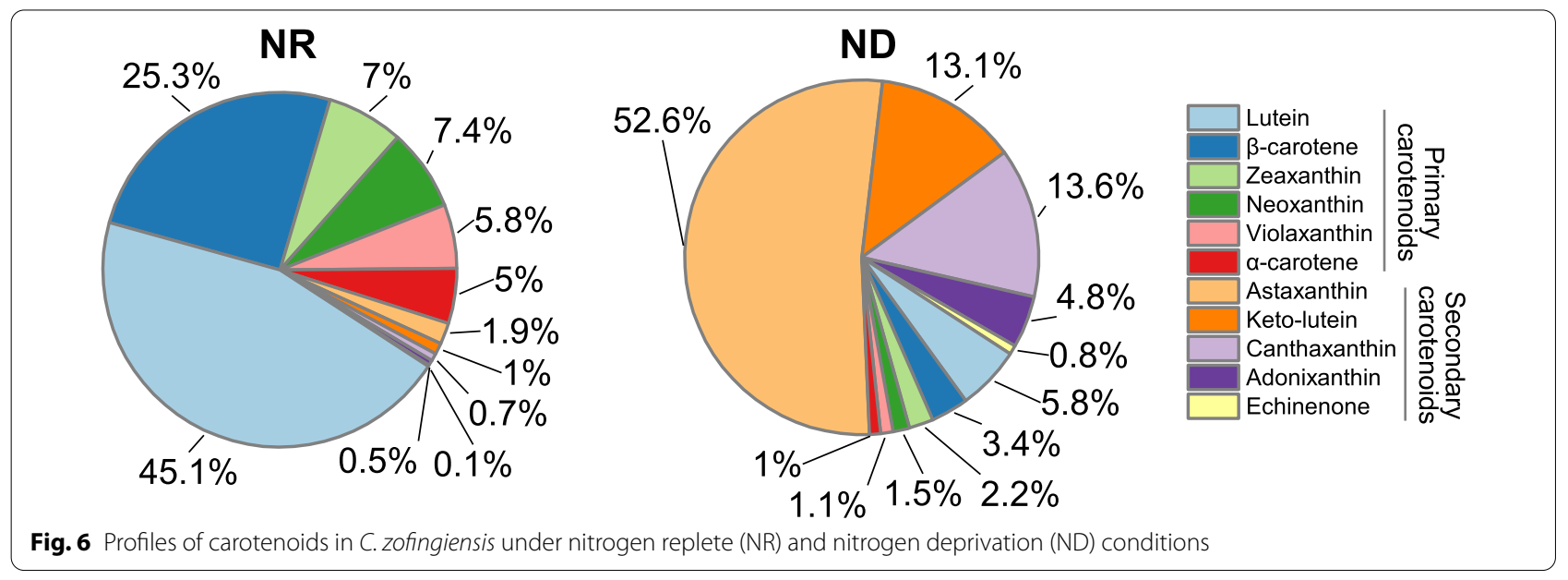




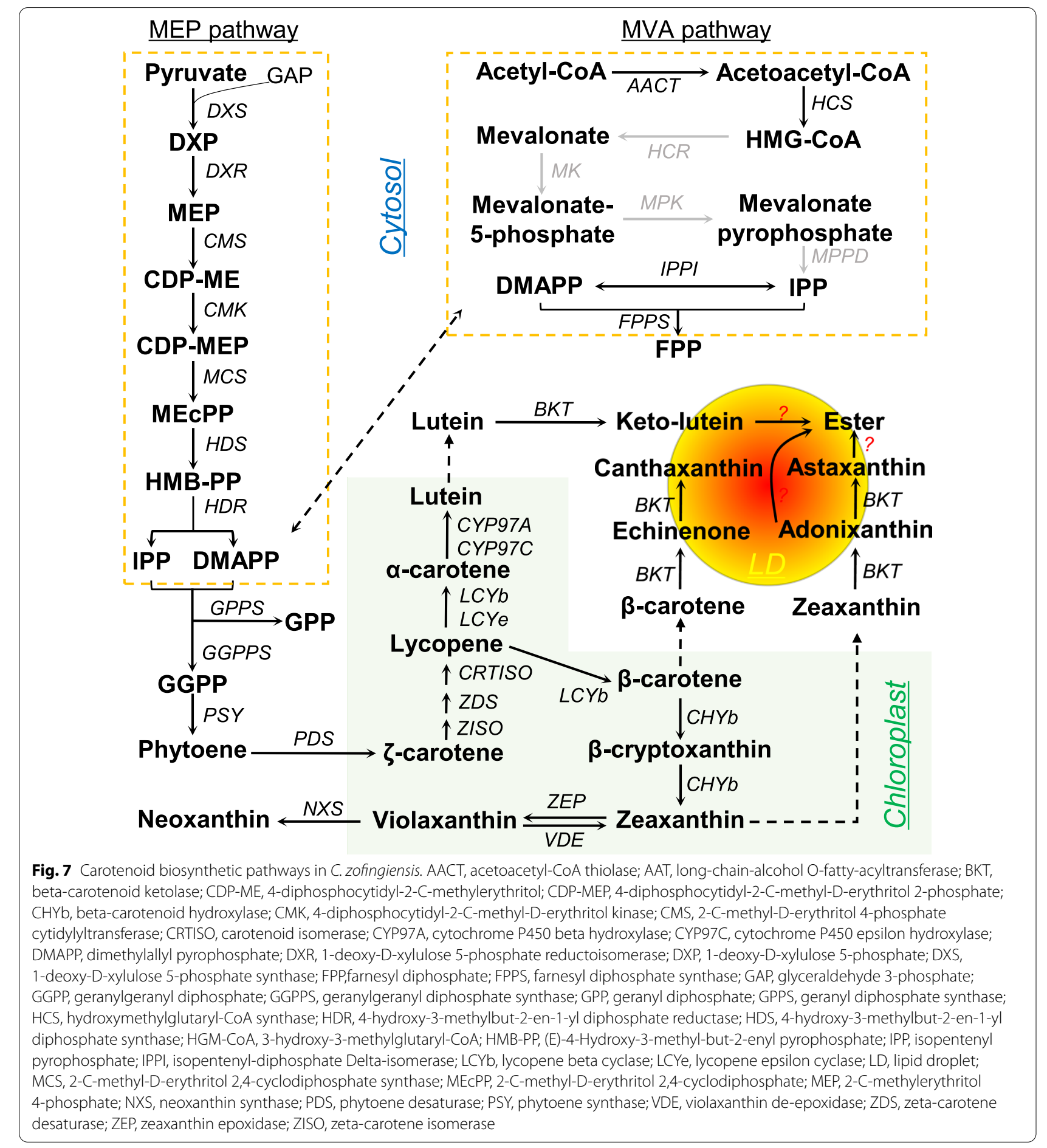

\section{IPP/DMAPP formation}

There are two pathways for the biosynthesis of IPP/ DMAPP in vascular plants, the 2-C-methylerythritol 4-phosphate (MEP) pathway and mevalonate (MVA) pathway [229]. The MEP pathway occurs in the chloroplast and converts pyruvate and glyceraldehyde 3-phosphate (GAP) to IPP/DMAPP via the intermediates 1-deoxy-D-xylulose 5-phosphate (DXP), MEP, 4-diphosphocytidyl-2-C-methylerythritol (CDP-ME), 4-diphosphocytidyl-2-C-methyl-D-erythritol 2-phosphate (CDP-MEP), 2-C-methylD-erythritol 2,4-cyclodiphosphate (MEcPP), and 
Table 5 Putative genes involved in carotenoid biosynthesis in C. zofingiensis

\begin{tabular}{|c|c|c|c|c|}
\hline \multirow{2}{*}{$\begin{array}{l}\text { Gene name } \\
\text { abbreviations }\end{array}$} & \multicolumn{2}{|l|}{ Gene ID } & \multirow[t]{2}{*}{ Gene description } & \multirow[t]{2}{*}{ References } \\
\hline & JGI v5.2.3.2 & GenBank & & \\
\hline \multicolumn{5}{|c|}{ IPP/DMAPP synthesis } \\
\hline DXS & Cz02g35280 & MT188717 & 1-deoxy-D-xylulose 5-phosphate synthase & {$[41]$} \\
\hline DXR & Cz07g19130 & MT188718 & 1-deoxy-D-xylulose 5-phosphate reductoisomerase & {$[41]$} \\
\hline CMS & Cz12g10090 & MT188719 & 2-C-methyl-D-erythritol 4-phosphate cytidylyltransferase & {$[41]$} \\
\hline CMK & Cz11g24270 & MT188720 & 4-diphosphocytidyl-2-C-methyl-D-erythritol kinase & {$[41]$} \\
\hline MCS & Cz02g10180 & MT188721 & 2-C-methyl-D-erythritol 2,4-cyclodiphosphate synthase & {$[41]$} \\
\hline HDS & Cz13g08020 & MT188722 & 4-hydroxy-3-methylbut-2-en-1-yl diphosphate synthase & {$[41]$} \\
\hline HDR & Cz05g23010 & MT188723 & 4-hydroxy-3-methylbut-2-enyl diphosphate reductase & {$[41]$} \\
\hline AACT & Cz04g25080 & MT188724 & Acetoacetyl-CoA thiolase & {$[41]$} \\
\hline HCS & Cz19g02060 & MT188725 & Hydroxymethylglutaryl-CoA synthase & [41] \\
\hline IPPI1 & Cz03g02150 & & Isopentenyl-diphosphate delta-isomerase & [41] \\
\hline IPPI2 & Cz08g23110 & & Isopentenyl-diphosphate delta-isomerase & [41] \\
\hline IPPI3 & Cz03g10110 & & Isopentenyl-diphosphate delta-isomerase & [41] \\
\hline \multicolumn{5}{|c|}{ IPP/DMAPP to carotenoids } \\
\hline GPPS & Cz02g31110 & & Geranyl diphosphate synthase & \\
\hline FPPS & Cz01g03190 & & Farnesyl diphosphate synthase & \\
\hline GGPPS & Cz02g19200 & & Geranylgeranyl diphosphate synthase & \\
\hline PSY & Cz05g32220 & FR670783 & Phytoene synthase & [239] \\
\hline PDS & Cz02g32280 & EF621406 & Phytoene desaturase & [245] \\
\hline ZDS & Cz10g17010 & & $\zeta$-carotene desaturase & \\
\hline ZISO & Cz10g17130 & & $\zeta$-carotene isomerase & \\
\hline CRTISO1 & Cz16g01210 & & Carotenoid isomerase & \\
\hline CRTISO2 & Cz12g03260 & & Carotenoid isomerase & \\
\hline CRTISO3 & Cz14g22040 & & Carotenoid isomerase & \\
\hline LCYe & Cz09g18310 & HE664109 & Lycopene $\varepsilon$-cyclase & {$[250]$} \\
\hline LCYb & Cz12g10170 & FN563998 & lycopene $\beta$-cyclase & [251] \\
\hline CYP97A1 & Cz13g16110 & & Cytochrome P450 $\beta$-hydroxylase & \\
\hline CYP97A2 & Cz09g14130 & & Cytochrome P450 $\beta$-hydroxylase & \\
\hline CYP97C & Cz09g07100 & & Cytochrome P450 ع-hydroxylase & \\
\hline $\mathrm{CHYb}$ & Cz12g16080 & EU016205 & $\beta$-carotenoid hydroxylase & [304] \\
\hline BKT1 & Cz13g13100 & AY772713 & $\beta$-carotenoid ketolase/oxygenase & {$[260]$} \\
\hline BKT2 & Cz04g11250 & & $\beta$-carotenoid ketolase/oxygenase & \\
\hline ZEP & Cz07g30060 & HE863825 & Zeaxanthin epoxidase & [252] \\
\hline VDE & Cz06g02070 & & Violaxanthin de-epoxidase & \\
\hline NXS & Cz15g04070 & & Neoxanthin synthase & \\
\hline
\end{tabular}

(E)-4-Hydroxy-3-methyl-but-2-enyl pyrophosphate (HMB-PP), catalyzed in order by DXP synthase (DXS), DXP reductoisomerase (DXR), 2-C-methyl-D-erythritol 4-phosphate cytidylyltransferase (CMS), 4-diphosphocytidyl-2-C-methyl-D-erythritol kinase (CMK), 2-C-methyl-D-erythritol 2,4-cyclodiphosphate synthase (MCS), 4-hydroxy-3-methylbut-2-en-1-yl diphosphate synthase (HDS), and 4-hydroxy-3-methylbut-2-en-1-yl diphosphate reductase (HDR). By contrast, the MVA pathway occurs in the cytosol and starts with acetylCoA for producing IPP/DMAPP via the intermediates
acetoacetyl-CoA, 3-hydroxy-3-methylglutaryl-CoA (HGM-CoA), mevalonate, mevalonate-5-phosphate, and mevalonate pyrophosphate, catalyzed successively by acetoacetyl-CoA thiolase (AACT), hydroxymethylglutaryl-CoA synthase (HCS), HMG-CoA reductase (HCR), mevalonate-5-kinase (MK), phosphomevalonate kinase (MPK), and mevalonate-5-pyrophosphate decarboxylase (MPPD). IPP and DMAPP can be interconverted by the action of IPP delta-isomerase (IPPI).

All enzymes involved in the MEP pathway have been identified in C. zofingiensis and each are encoded by a 
sing-copy gene; by contrast, many enzymes involved in the MVA pathway are missing (Fig. 7 and Table 5). Similarly, the MVA pathway is also incomplete in the green algae $C$. reinhardtii and $H$. pluvialis [230, 231], suggesting that it may be lost during the evolution of green algae [232]. Moreover, it is believed that C. reinhardtii and $H$. pluvialis utilize the MEP pathway rather than the MVA pathway to supply IPP/DMAPP for carotenoid biosynthesis [231, 233]. Fosmidomycin and mevinolin are inhibitors targeting the MEP pathway and the MVA pathway, respectively. Carotenoid levels in C. zofingiensis were impaired by fosmidomycin instead of mevinolin, indicating that this alga also employs the MEP pathway for carotenoid biosynthesis [14]. Intriguingly, upon ND or SD that triggers accumulation of secondary carotenoids including astaxanthin, the MEP pathway was not up-regulated at the transcriptional level in C. zofingiensis [39, 41]. Probably, no up-regulation of the MEP pathway is needed to provide precursors for carotenoids, as the level of total carotenoids in C. zofingiensis shows little change. By contrast, in $H$. pluvialis the MEP pathway showed a considerable up-regulation in response to ND and/or HL $[230,234]$. This difference may partially explain why $C$. zofingiensis synthesizes a lower level of astaxanthin than H. pluvialis.

\section{Biosynthesis of primary carotenoids}

Condensation of one DMAPP with one, two and three IPP molecules produces geranyl diphosphate (GPP), farnesyl diphosphate (FPP) and geranylgeranyl diphosphate (GGPP), which are catalyzed by GPP synthase (GPPS), FPP synthase (FPPS) and GGPP synthase (GGPPS), respectively. GGPP is the direct metabolic precursor for carotenoids. The head-to-head condensation of two GGPP molecules mediated by phytoene synthase (PSY) leads to formation of phytoene, a colorless C40 carotenoid. Phytoene is then converted to lycopene through several desaturation and isomerization steps catalyzed by phytoene desaturase (PDS), $\zeta$-carotene isomerase (ZISO), $\zeta$-carotene desaturase (ZDS) and carotenoid isomerase (CRTISO) (Fig. 7). It is worth noting that some photosynthetic bacteria, differing from vascular plants and eukaryotic algae, employ a single enzyme, crtI, to catalyze the formation of lycopene from phytoene [235-237]. C. zofingiensis harbors a single gene for each of GPPS, FPPS, GGPPS, PSY, PDS, ZISO and ZDS and three gene copies for CRTISO (Table 5). PSY is considered as the first and key rate-limiting enzyme that determines the metabolic flux to carotenoids [238]. Heterologous expression of the C. zofingiensis PSY gene in C. reinhardtii led to increased level of carotenoids [239], consistent with previous studies of overexpressing PSY gene in algae and vascular plants [238, 240, 241]. PDS that catalyzes the desaturation of phytoene to $\zeta$-carotene is also considered as a rate-limiting enzyme for carotenoid biosynthesis [242, 243]. In C. zofingiensis, PDS was up-regulated under carotenogenic conditions of HL and correlated with carotenoid accumulation [244, 245]. The up-regulation of PDS by HL also occurs in H. pluvialis, not only at the transcriptional level but also at the translational level [246]. It has been reported that overexpression of $P D S$ promoted carotenoid synthesis in several algae including C. zofingiensis [34], H. pluvialis [243] and C. reinhardtii [247]. Besides, PDS mutants with certain point mutations showed strong resistance to the herbicide norflurazon and can be used as dominant selectable marker for algal transformation [34, 243, 247, 248]. Interestingly, the mutation of $\mathrm{L}$ (leucine) to $\mathrm{F}$ (phenylalanine) at the position 516 of C. zofingiensis PDS, unlike other mutations that confer norflurazon resistance yet attenuate desaturation activity, enhanced the desaturation activity by $30 \%$ [249].

The cyclization of lycopene is critical as it determines the destination of lycopene to either $\beta$-carotene or $\alpha$-carotene and their downstream derivatives. The action of lycopene $\beta$-cyclase ( $\mathrm{LCYb}$ ) adds $\beta$-ionone rings on both ends of lycopene leading to $\beta$-carotene formation, while the collaboration of LCYb and lycopene $\varepsilon$-cyclase (LCYe) generates a $\beta$-ionone ring on one end and a $\varepsilon$-ionone ring on the other end resulting in $\alpha$-carotene formation (Fig. 7). C. zofingiensis harbors a single gene for each of LCYb and LCYe; LCYb can convert lycopene and $\delta$-carotene to $\beta$-carotene and $\alpha$-carotene, respectively, while LCYe only acts on lycopene to produce $\delta$-carotene $[250,251]$. These two genes have differential expression patterns, e.g., $L C Y b$ is up-regulated, while $L C Y e$ is considerably down-regulated in response to stress conditions that trigger accumulation of $\beta$-carotene derivatives at the expense of $\alpha$-carotene derivatives [18, 32, 39, 250, 252], supporting the determining roles of LCYb and LCYe in allocating carotenoid flux between the two branching ways.

Catalyzed by the non-heme di-iron type of $\beta$-carotenoid hydroxylase $(\mathrm{CHYb}), \beta$-carotene undergoes two sequential hydroxylation steps leading to zeaxanthin formation via the intermediate $\beta$-cryptoxanthin. By contrast, the hydroxylation of $\alpha$-carotene to lutein is mediated by the heme-containing cytochrome P450 enzymes CYP97A and CYP97C, which add a hydroxyl group on the $\beta$ - and $\varepsilon$-rings of $\alpha$-carotene, respectively (Fig. 7). Of the four hydroxylase genes in C. zofingiensis (one $\mathrm{CHYb}$ gene, two CYP97A genes and one CYP97C genes), only $C H Y b$ has been functionally characterized [14, 253]. Unlike $C H Y b$ that varies in its expression pattern depending on stress conditions, CYP97A and CYP97C genes are normally down-regulated, in support of attenuated 
lutein accumulation in C. zofingiensis [18, 32, 39, 244]. Zeaxanthin, by the action of zeaxanthin epoxidase (ZEP) that introduces epoxy groups, is converted to violaxanthin via the intermediate antheraxanthin. Violaxanthin can also be converted back to zeaxanthin by violaxanthin de-epoxidase (VDE). The interconversion between zeaxanthin and violaxanthin is referred to as the violaxanthin cycle, which is widely present in vascular plants and algae and plays important roles in photoprotection against adverse environments [254, 255]. Moreover, the introduction of an allenic double bond to violaxanthin generates neoxanthin, which is mediated by neoxanthin synthase (NXS). There is a single gene present in C. zofingiensis encoding for each of ZEP, VDE and NXS (Table 5). These genes tend to undergo transcriptional suppression upon stress conditions, consistent with the impaired synthesis of violaxanthin and neoxanthin $[18$, 32, 39].

\section{Astaxanthin biosynthesis}

Unlike the primary carotenoids mentioned above, astaxanthin is a keto-carotenoid and its formation requires additional ketolation steps mediated by $\beta$-carotenoid ketolase (BKT) in algae $[111,256]$. The biosynthesis of astaxanthin from $\beta$-carotene, involving two hydroxylation steps and two ketolation steps in total, has multiple routes and may vary in different organisms. Considering that BKT is efficient in converting $\beta$-carotene to canthaxanthin but poor in converting zeaxanthin to astaxanthin and $\mathrm{CHYb}$ has strong activity to catalyze astaxanthin formation from canthaxanthin [253, 257-259], H. pluvialis is likely to employ the route with two stepwise ketolation reactions followed by two stepwise hydroxylation reactions as the major contributor for astaxanthin synthesis. $C$. zofingiensis is predicted to contain two $B K T$ genes, $B K T 1$ and $B K T 2$, yet only $B K T 1$ has been functionally characterized [228, 260]. Intriguingly, inactivation of $B K T 1$ led to complete abolishment of astaxanthin accumulation [33, 261], indicating that $B K T 1$ instead of $B K T 2$ is involved in astaxanthin biosynthesis in C. zofingiensis. Differing from $H$. pluvialis that contains only a trace amount of canthaxanthin, C. zofingiensis accumulates canthaxanthin up to $30 \%$ of the secondary carotenoids $[13,19,22,54,55]$, indicating that its CHYb may have no or low activity in converting canthaxanthin to astaxanthin thus leading to the buildup of canthaxanthin as an end product. On the other hand, C. zofingiensis synthesizes adonixanthin, the intermediate of ketolating zeaxanthin to astaxanthin that is not detectable in $H$. pluvialis, and adonixanthin is stimulated to accumulate upon astaxanthin-inducing conditions [18, 19, 32, 39]. Moreover, suppression of BKT activity by the specific chemical inhibitor diphenylamine or BKT1 mutation boosts zeaxanthin accumulation at the expense of astaxanthin [33, 261, 262]. These results, plus the functional validation of $B K T 1$ in a zeaxanthin-producing $E$. coli system [263], suggesting that the $C$. zofingiensis BKT accepts zeaxanthin as the substrate to form astaxanthin with a moderate efficiency. In line with these studies, the in vitro assays of C. zofingiensis BKT and CHYb provided solid evidence to support that BKT1 is able to ketolating zeaxanthin to astaxanthin, while $\mathrm{CHYb}$ has no activity in hydroxylating canthaxanthin to astaxanthin [14]. In this context, C. zofingiensis employs a route different from $H$. pluvialis for astaxanthin synthesis, namely, the $\mathrm{CHYb}$ catalyzed hydroxylation of $\beta$-carotene to zeaxanthin first and then the BKT-catalyzed ketolation of zeaxanthin to astaxanthin (Fig. 7). It is worth noting that C. zofingiensis BKT1 may also act on lutein and adds a keto group on the $\beta$-ring to generate keto-lutein, as $B K T 1$ dysfunction impairs keto-lutein accumulation [261]. By contrast, no keto-lutein is detected in $\mathrm{H}$. pluvialis.

The amino acid variance in certain positions of the BKT polypeptides may cause the functional difference of BKT enzymes between $C$. zofingiensis and $H$. pluvialis. It has been reported that singe-amino acid mutations in over ten positions of C. zofingiensis BKT1 abolished astaxanthin accumulation [33, 36, 261]. One of these mutated positions, R51 (arginine at the position 51), may be critical for C. zofingiensis BKT1 in the function of ketolating zeaxanthin to astaxnathin [14]. First, in the corresponding position of $H$. pluvialis BKT that has no activity on zeaxanthin ketolation, the amino acid residue is lysine (K), different from C. zofingiensis BKT1. Second, substitution of R51 with $\mathrm{K}$ in C. zofingiensis BKT1 blocks astaxanthin accumulation and promotes zeaxanthin level considerably [36]. Third, the BKT from C. reinhardtii, which resembles $C$. zofingiensis BKT1 and functions in converting zeaxanthin to astaxanthin [263], harbors the amino acid residue $\mathrm{R}$ in the position corresponding to R51 of C. zofingiensis BKT1. Moreover, in this position, C. zofingiensis BKT2 that is believed to have no activity on zeaxanthin contains the same amino acid residue $\mathrm{K}$ as $H$. pluvialis BKT. Functional validation of the K-to- $\mathrm{R}$ mutant of $H$. pluvialis BKT remains to be performed and would provide insights into understanding the substrate utilization of BKT enzymes for zeaxanthin. The functional difference of $\mathrm{CHYb}$ enzymes between $C$. zofingiensis and $H$. pluvialis may be also attributed to the amino acid variance in their polypeptides. It has been reported that overexpression of $C r B K T$ in $C$. reinhardtii and the vascular plants Arabidopsis thaliana and Lycopersicon esculentum each results in the accumulation of a substantial amount of canthaxanthin [253, 263, 264]. Therefore, the endogenous $\mathrm{CHYb}$ enzymes from these organisms are likely similar to C. zofingiensis $\mathrm{CHYb}$ and have no/ 
low activity in converting canthaxanthin to astaxanthin. In silico analysis of these $\mathrm{CHYb}$ polypeptides with $H$. pluvialis $\mathrm{CHYb}$ has suggested involvement of several candidate amino acid residues in the function of ketolating canthaxanthin to astaxanthin [14].

Similar in H. pluvialis, astaxanthin in C. zofingiensis is stored in cytoplasmic LDs $[14,109,265]$. As the primary carotenoids including lycopene, $\beta$-carotene and zeaxanthin are synthesized in the chloroplast, whereas the ketolation steps for astaxanthin biosynthesis occur outside of the chloroplast [108], certain carotenoids have to transport across the chloroplast envelops for supporting extrachloroplastic astaxanthin synthesis. It is believed that in $H$. pluvialis the transport takes place after $\beta$-ionone ring cyclization, namely, $\beta$-carotene is the intermediate exported from the chloroplast during astaxanthin induction [109]. The exported $\beta$-carotene is likely packed into cytoplasmic LDs and undergoes ketolation and hydroxylation steps for astaxanthin biosynthesis, considering that both activities of $\mathrm{BKT}$ and $\mathrm{CHYb}$ are detected in the isolated LD fractions [108]. This may not hold true in C. zofingiensis, as neither BKT nor $\mathrm{CHYb}$ is present in LDs based on the proteomics analysis of the purified LD fraction [40]. Albeit lacking experimental evidence, C. zofingiensis BKT and $\mathrm{CHYb}$ are predicted to reside in the ER and chloroplast, respectively [41]. In this context, export of both $\beta$-carotene and zeaxanthin from the chloroplast is in need to support the BKT-mediated ketolation for producing canthaxanthin and astaxanthin, respectively. Nevertheless, if the $\mathrm{CHYb}$ activity is also present outside the chloroplast in C. zofingiensis, zeaxanthin export may be not necessary. As no signs of vesicular transport observed, it has been hypothesized that carotenoid binding proteins rather than vesicular transport are involved in facilitating export of $\beta$-carotene in $H$. pluvialis $[109,246]$. Nevertheless, no such protein has so far been identified. In algae under stress conditions, LDs are connected with both the chloroplast and ER and may serve as bridges to allow diffusion of lipids, such as DAG between the chloroplast and ER along the LD-delimiting mono-layer [266]. This may also be applicable to carotenoids in $C$. zofingiensis, for example, $\beta$-carotene and zeaxanthin are translocated along the LD mono-layer to ER for ketolation mediated by BKT; the ketolated carotenoids, such as astaxanthin, canthaxanthin and adonixanthin, can diffuse as well along the LD mono-layer and enter LDs for storage.

\section{Esterification of astaxanthin}

Astaxanthin in C. zofingiensis and $H$. pluvialis has long been found to be present mainly in the form of ester (mono-ester and di-ester), which reaches up to $90 \%$ of total astaxanthin depending on algal strains and culture conditions $[13,14,16,88,267]$. It is thought that the formation of astaxanthin ester from free astaxanthin involves acyltransferase(s) that may transfer an acyl moiety from acyl-CoA and/or acyl-containing lipids to the hydroxyl end groups of astaxanthin. Nevertheless, the enzyme(s) responsible for esterification of astaxanthin have yet to be identified, albeit there have been several presumptions. In mammals, DGAT1, besides the involvement in TAG synthesis, has been demonstrated to also possess acyl CoA:retinol acyltransferase activity and catalyze retinol esterification [268, 269]. Retinol is a degradation production of carotenoids, raising the hypothesis whether DGAT(s) have the ability to esterify astaxanthin. Based on the results that the ER fraction (where DGAT enzymes reside) can mediate astaxanthin ester synthesis by feeding $\beta$-carotene in vitro and the addition of DGAT inhibitors impair astaxanthin ester formation, DGATs have been proposed as the candidate enzymes responsible for astaxanthin esterification in $H$. pluvialis [15]. However, it cannot be excluded that unknown acyltransferases that have astaxanthin esterification activity may also be present in the ER fraction and vulnerable to DGAT inhibitors. It is worth noting that in C. reinhardtii and some vascular plants, albeit multiple DGATs are present in their genomes, the reconstruction of astaxanthin biosynthesis pathways in them leads to the accumulation of free astaxanthin rather than ester [263, 264, $270,271]$, questioning the role of DGATs in astaxanthin esterification. Since both C. zofingiensis and H. pluvialis harbor multiple $D G A T$ gene copies yet lack well-established genetic tools $[189,192,193]$, it is challenging to validate the esterification function of these DGAT genes in vivo via genetic manipulations. Functional analysis in free astaxanthin-producing yeast may represent an option. Recently, heterologous expression of the ten $C$. zofingiensis DGAT genes each in a free astaxanthin-producing yeast strain has been conducted and the results failed to support the role of DGATs in astaxanthin esterification [14]. Another proposed candidate enzyme for astaxanthin esterification is a long-chain-alcohol O-fatty-acyltransferase from C. zofingiensis [33], which is transcriptionally up-regulated under many astaxanthin inducing conditions [14, 32]. Nevertheless, heterologous expression of this gene in the free astaxanthin-producing yeast strain also failed to produce detectable esterified astaxanthin [14].

Moreover, it has been reported in vascular plants that esterase-like enzymes are involved in esterification of several carotenoids. One is PYP1, an esterase/lipase/ thioesterase family of acyltransferase from tomato that contributes to esterification of violaxanthin and neoxanthin [272]. The other one is XAT, a Gly-Asp-Ser-Leu motif-containing esterase/lipase, which has the ability 
to esterify lutein, zeaxanthin and cryptoxanthin using a broad range of acyl donors [273]. They may have the potential to also function as astaxanthin esterase. Searching C. zofingiensis genome reveals the presence of homolog of PYP1 (encoded by Cz02g16070) but not of XAN. Experimental evidence is needed for clarifying function of this PYP1 homolog. On the other hand, a high-throughput forward genetic screening via random mutagenesis represents an alternative option to probe the genuine acyltransferase responsible for astaxanthin esterification. Although labor-intensive and time-consuming, it has been successfully applied to C. zofingiensis for identifying genes involved in astaxanthin biosynthesis and lipid metabolism [33, 35, 36, 261].

Interestingly, it has been reported that some of the astaxanthin-producing algae accumulate only free astaxanthin $[105,122]$, raising the questions that why $C$. zofingiensis and $H$. pluvialis synthesize predominantly esterified astaxanthin, whereas some algae produce only free astaxanthin and what's the biological significance of astaxanthin esterification. Identification and characterization of the genuine astaxanthin esterase and mutants of algae defective in this enzyme would help address these questions.

\section{Mechanistic insights into carotenogenesis for astaxanthin biosynthesis in C. zofingiensis}

As mentioned above, C. zofingiensis and H. pluvialis tend to synthesize and accumulate astaxanthin under stress conditions. It is widely accepted that astaxanthin formation is a survival strategy of algae to cope with adverse conditions [56, 111]. Astaxanthin biosynthesis may offer multiple layers of protection to C. zofingiensis cells. First, astaxanthin accumulates in cytoplasmic LDs that reside peripherally and surround the chloroplast $[14,55]$. These astaxanthin-containing LDs may function like a sunscreen to reduce the amount of light impinging on the chloroplast and other organelles, thus attenuating photosynthetic photoinhibition and photodamage associated with excess photons. Second, C. zofingiensis accumulates reactive oxygen species (ROS) triggered by stress conditions [13, 244]; astaxanthin has strong antioxidation activity and can serve as a powerful scavenger to mitigate excess ROS for preventing algal cells from damage. Third, astaxanthin is more abundant in oxygen content than other carotenoids in C. zofingiensis; astaxanthin buildup has the potential to lower intracellular oxygen levels and thus the generation of ROS.

Exposure of $C$. zofingiensis to astaxanthin inducing conditions, secondary carotenoids increased considerably, yet the content of total carotenoids showed only a slight increase accompanied with a severe decrease of primary carotenoids $[14,18,32,41]$. In this context, the increase of secondary carotenoids including astaxanthin in C. zofingiensis is not likely attributed to the enhancement of overall carotenoid flux, as suggested in $\mathrm{H}$. pluvialis, but instead caused by rerouting the carotenoid flux from primary carotenoids to secondary carotenoids. This is also supported by the transcriptional regulation of carotenoid biosynthetic pathways in C. zofingiensis upon stress conditions: the MEP pathway and lycopene formation from IPP/DMAPP were not stimulated, while genes involved in the biosynthesis of astaxanthin and other secondary carotenoids are up-regulated and genes involved in lutein biosynthesis were down-regulated [18, 32, 39, 41]. It is worth noting that stress conditions that induce astaxanthin biosynthesis also trigger ROS buildup in $C$. zofingiensis [13, 244]. Moreover, addition of external ROS to C. zofingiensis cultures can promote accumulation of secondary carotenoids including astaxanthin [113, 244]. These also happen in H. pluvialis [274-276]. Therefore, it is believed that ROS are involved in the regulation of carotenogenesis for astaxanthin biosynthesis. Nevertheless, what ROS species are generated by these stress conditions and how algal cells sense ROS for triggering carotenogenesis still remain largely unknown.

C. zofingiensis synthesizes astaxanthin, yet at a level much lower than that in H. pluvialis, likely attributed to the differences between the two algae with respect to astaxanthin biosynthesis and regulation. First, during carotenogenesis, up-regulation of the MEP pathway and lycopene formation from IPP/DMAPP occurs in $H$. pluvialis but not in C. zofingiensis [41]. Therefore, the carbon flux to carotenoids is limited and cannot support high astaxanthin accumulation in C. zofingiensis. Second, unlike $H$. pluvialis $\mathrm{BKT}$ and $\mathrm{CHYb}$ that have strong activity in converting $\beta$-carotene in succession to astaxanthin without accumulating intermediates, $C$. zofingiensis $\mathrm{BKT}$ is able to convert zeaxanthin to astaxanthin yet not efficiently and $\mathrm{CHYb}$ has no activity in hydroxylating canthaxanthin to astaxanthin, leading to buildup of the intermediates canthaxanthin and adonixanthin $[14,36]$. In this context, the astaxanthin biosynthetic pathway in C. zofingiensis performs less efficiently than that in $H$. pluvialis. Third, aside from acting on $\beta$-carotene and zeaxanthin, $C$. zofingiensis BKT is likely able to convert lutein to keto-lutein for accumulation [14, 36], further diverting carotenoid flux away from astaxanthin. Fourth, the synthesis of violaxanthin competes with astaxanthin formation for the substrate zeaxanthin in $C$. zofingiensis and thus may attenuate zeaxanthin availability for astaxanthin synthesis. Fifth, the astaxanthin esterase in C. zofingiensis is likely to accept more substrates for esterification than that in $H$. pluvialis, giving that $C$. zofingiensis accumulates esterified forms of astaxanthin, adonixanthin and keto-lutein $[41,107]$, while $H$. pluvialis 
produces only esterified astaxanthin [267, 277]. The nonspecific substrate utilization of astaxanthin esterase may impair the availability of the enzyme for astaxanthin ester formation in C. zofingiensis. The less efficient esterification of astaxanthin likely in turn inhibits astaxanthin synthesis in a feedback manner.

\section{Crosstalk between TAG and astaxanthin biosynthesis in C. zofingiensis}

Astaxanthin is a secondary metabolite that accumulates in $C$. zofingiensis and $H$. pluvialis under diverse stress conditions $[13,15,16,19,32,230]$. These stress conditions also trigger synthesis of TAG, the major storage lipid, in the two algae. The concurrent synthesis of astaxanthin and TAG that share and may compete for the carbon precursor pyruvate, plus the presence of astaxanthin predominantly esterified with fatty acids, points to the potential crosstalk between TAG and astaxanthin biosynthesis in $C$. zofingiensis and $H$. pluvialis. It has been demonstrated that inhibition of de novo fatty acid synthesis by specific chemical inhibitors attenuated or even abolished astaxanthin accumulation in $H$. pluvialis [15, 16, 278]. Probably, the inhibition of fatty acid synthesis causes a shortage of fatty acids for astaxanthin esterification leading to attenuated accumulation of astaxanthin ester in H. pluvialis [278]. Furthermore, astaxanthin, once synthesized, is packed into the TAG-filled LDs for storage [109]. The inhibition of fatty acid synthesis causes a considerable reduction in TAG level and thus less LDs for accommodating astaxanthin, which likely in turn imposes feedback inhibition on astaxanthin synthesis and esterification. Therefore, it has been proposed that a certain level of TAG (or a certain number of LDs) is a prerequisite for astaxanthin synthesis and accumulation in H. pluvialis [15, 16, 278].

Intriguingly, the impaired astaxanthin accumulation caused by inhibition of de novo fatty acid synthesis that happens in $H$. pluvialis does not occur in C. zofingiensis; instead, astaxanthin showed an increase upon treatment of the inhibitor cerulenin [13, 14, 279]. The astaxanthin increase associated with cerulenin treatment is likely from transformation of other carotenoids rather than the shunt of carbon flux from fatty acids to carotenoid biosynthetic pathways, as the total carotenoids showed little change, whereas $\beta$-carotene and canthaxanthin decreased [14]. One possible explanation for the contrary responses of astaxanthin to cerulenin treatment in $C$. zofingiensis and $H$. pluvialis is that the former synthesizes considerably lower astaxanthin than the latter and, therefore, needs fewer fatty acids and TAG-filled LDs for astaxanthin esterification and storage, respectively. Consistent with this, C. zofingiensis has lower ratios of astaxanthin/total fatty acids (TFA) and astaxanthin/TAG than $H$. pluvialis
[14]. Nevertheless, both ratios in C. zofingiensis showed drastic increases upon cerulenin treatment and their values exceeded that in $H$. pluvialis, suggesting astaxanthin biosynthesis and accumulation in C. zofingiensis may not be restricted by the availability of fatty acids or TAG [14]. Probably, cerulenin treatment induces ROS production [13], which in turn serves as a signal to stimulate astaxanthin biosynthesis in C. zofingiensis.

The inhibition of astaxanthin biosynthesis, on the other hand, has little effect on TAG accumulation in C. zofingiensis [14]. This resembles the observations in H. pluvialis [16] and points to the fact that TAG biosynthesis is independent of astaxanthin biosynthesis process in these two algae. It is reasonable as many algae that do not synthesize astaxanthin are also able to accumulate TAG [3].

\section{Metabolic engineering for potential improvements in TAG and astaxanthin accumulation by $C$. zofingiensis}

Both TAG and astaxanthin are secondary metabolites and generally accumulate in C. zofingiensis under stress conditions rather than favorable growth conditions. These abiotic stress conditions, nevertheless, impair algal growth and thus the production of TAG and astaxanthin. Genetic engineering of C. zofingiensis has the potential to allow the alga to synthesize more target products and accumulate even under non-stress conditions. Many candidate genes with engineering potential for improving TAG and/or astaxanthin production have been identified, which can be achieved by such strategies as 'pushing, 'pulling' and 'protection' summarized in Table 6.

\section{Metabolic engineering for TAG improvement}

Acetyl-CoA is the precursor of de novo fatty acid synthesis and increasing acetyl-CoA supply has proven to be a feasible 'pushing' strategy for promoting fatty acid synthesis and TAG accumulation in several algae [280-282]. C. zofingiensis mainly employs the chloroplastic PDHC and ACS, which are transcriptionally up-regulated by ND, to produce acetyl-CoA for de novo fatty acid synthesis. Overexpression of them has the potential to enhance TAG synthesis in C. zofingiensis. The fatty acyls used in the Kennedy pathway for TAG assembly are in the form of acyl-CoAs; they can be converted from the de novo synthesized acylACPs mediated by the combination of FAT and LACS or from turnover of membrane lipids mediated by the combination of membrane lipid lipase and LACS. In C. zofingiensis, FAT, LACS2, PGD1 and certain other putative membrane lipid lipase genes (Cz02g15090, Cz03g14190, Cz01g06170 and Cz12g10010) are considerably up-regulated by TAG inducing conditions and may represent promising engineering targets. As 
Table 6 List of potential engineering targets for enhancing the synthesis of TAG and/or astaxanthin in C. zofingiensis

\begin{tabular}{|c|c|c|c|c|}
\hline Gene Name & Gene ID & Function description & $\begin{array}{l}\text { Expression } \\
\text { pattern upon } \\
\text { ND }\end{array}$ & Strategies \\
\hline \multicolumn{5}{|c|}{ Metabolic engineering for TAG improvements } \\
\hline $\mathrm{PDHC}$ & $\begin{array}{l}\text { E1A (Cz03g08090), E1B } \\
\quad(C z 01 g 37230)\end{array}$ & Produces acetyl-CoA from pyruvate & Up-regulated & Overexpression \\
\hline ACS & ACS2 (Cz12g10100) & Produces acetyl-CoA from acetate & Up-regulated & Overexpression \\
\hline FAT & Cz04g05080 & Releases FFAs from acyl-ACPs & Up-regulated & Overexpression \\
\hline PGD1 & Cz01g38020 & Releases FFAs from MGDG & Up-regulated & Overexpression \\
\hline Putative membrane lipid lipases & $\begin{array}{l}\text { Cz02g15090, Cz03g14190, } \\
\text { Cz01g06170, } \\
\text { Cz12g10010 }\end{array}$ & Releases FFAs from membrane lipids & Up-regulated & Overexpression \\
\hline LACS & LACS2 (Cz1 1g20120) & Converts FFAs to acyl-CoAs for TAG synthesis & Up-regulated & Overexpression \\
\hline GPDH & GPDH2 (Cz04g17090) & Converts DHAP to G3P for TAG synthesis & Up-regulated & Overexpression \\
\hline GK & GK2 (Cz18g13070) & Converts glycerol to G3P for TAG synthesis & Up-regulated & Overexpression \\
\hline GPAT & GPAT2 (Cz09g31330) & $\begin{array}{l}\text { Transfers the acyl moiety from acyl-CoAs to G3P for } \\
\text { LPA synthesis }\end{array}$ & Up-regulated & Overexpression \\
\hline LPAAT & $\begin{array}{l}\text { LPAAT1 (Cz16g02090), } \\
\text { LPAAT2 (Cz04g14150) }\end{array}$ & $\begin{array}{l}\text { Transfers the acyl moiety from acyl-CoAs to LPA for } \\
\text { PA synthesis }\end{array}$ & Up-regulated & Overexpression \\
\hline DGAT & $\begin{array}{l}\text { DGAT1A (Cz06g05010), } \\
\text { DGTT5 (Cz09g08290) }\end{array}$ & $\begin{array}{l}\text { Transfers the acyl moiety from acyl-CoAs to DAG for } \\
\text { TAG synthesis }\end{array}$ & Up-regulated & Overexpression \\
\hline MLDP & Cz04g29220 & Stabilizes LDs for TAG storage & Up-regulated & Overexpression \\
\hline Putative TAG lipases & $\begin{array}{l}\text { SDP1 (Cz05g29160) } \\
\text { Cz05g31060 }\end{array}$ & Hydrolysis of TAG & Down-regulated & Suppression \\
\hline LACS & LACS5 (Cz12g27140) & Converts FFAs to acyl-CoAs for $\beta$-oxidation & Little change & Suppression \\
\hline \multicolumn{5}{|c|}{ Metabolic engineering for astaxanthin improvements } \\
\hline DXS & Cz02g35280 & Involved in the MEP pathway for IPP/DMAPP & Little change & Overexpression \\
\hline DXR & Cz07g19130 & Involved in the MEP pathway for IPP/DMAPP & Little change & Overexpression \\
\hline HDR & Cz05g23010 & Involved in the MEP pathway for IPP/DMAPP & Little change & Overexpression \\
\hline PSY & Cz05g32220 & Condenses GGPP to phytoene & Little change & Overexpression \\
\hline PDS & Cz02g32280 & Converts phytoene to phytofluene & Little change & Overexpression \\
\hline LCYb & Cz12g10170 & Synthesizes $\beta$-carotene from lycopene & Up-regulated & Overexpression \\
\hline LCYe & Cz09g18310 & Synthesizes a-carotene from lycopene & Down-regulated & Suppression \\
\hline BKT & BKT1 (Cz13g13100) & $\begin{array}{l}\text { Converts } \beta \text {-carotene and zeaxanthin to canthaxan- } \\
\text { thin and astaxanthin, respectively }\end{array}$ & Up-regulated & Overexpression \\
\hline $\mathrm{CHYb}$ & Cz12g16080 & Converts $\beta$-carotene to zeaxanthin & Up-regulated & Overexpression \\
\hline \multicolumn{5}{|c|}{ Metabolic engineering for both TAG and astaxanthin improvements } \\
\hline MYB & Cz10g24240, Cz06g23090 & $\begin{array}{l}\text { MYB transcriptional factor predicted to regulate } \\
\text { both TAG and astaxanthin synthesis }\end{array}$ & Up-regulated & Overexpression \\
\hline bHLH & Cz03g20070, UNPLg00160 & $\begin{array}{l}\text { bHLH transcriptional factor predicted to regulate } \\
\text { both TAG and astaxanthin synthesis }\end{array}$ & Up-regulated & Overexpression \\
\hline bZIP & Cz15g21170, UNPLg00449 & $\begin{array}{l}\text { bZIP transcriptional factor predicted to regulate } \\
\text { both TAG and astaxanthin synthesis }\end{array}$ & Up-regulated & Overexpression \\
\hline G6PD & Cz06g12080, Cz03g12030 & Provides NADPH & Up-regulated & Overexpression \\
\hline $6 P G D$ & Cz05g06160 & Provides NADPH & Up-regulated & Overexpression \\
\hline ME & Cz15g18140 & Produces pyruvate and NADPH from malate & Up-regulated & Overexpression \\
\hline
\end{tabular}

a support, heterologous expression of C. zofingiensis LACS2 in N. oceanica or yeast has proven to promote TAG synthesis [151]. Heterologous expression of Cz01g06170 or Cz12g10010 also promoted TAG synthesis in yeast [40]. G3P is the other precursor used for
TAG assembly and its generation can be either from glycerol catalyzed by glycerol kinase (GK) or from dihydroxyacetone phosphate (DHAP) catalyzed by G3P dehydrogenase (GPDH) [283]. C. zofingiensis GPDH2 and $G K 2$ correlate well with TAG accumulation at the 
transcriptional level and are candidate gene targets with engineering potential. It has been reported in $C$. reinhardtii that overexpression of GPD2, a homolog to C. zofingiensis GPDH2, promoted TAG accumulation substantially [284].

The acyltransferases GPAT, LPAAT and DGAT are appealing candidates of the 'pulling' strategy, as they provide strong pulling force to integrate fatty acids to the glycerol backbone for TAG assembly. It has been reported in several algae that overexpression of GPAT and/or $L P A A T$ allowed synthesis of more TAG [181, 285288]. In C. zofingiensis, GPAT2, LPAAT1 and LPAAT2 are stimulated to express under TAG inducing conditions and, therefore, are considered as promising gene targets. It has been demonstrated that engineering $C$. zofingiensis via overexpressing GPAT2 led to enhanced TAG accumulation [37]. Compared to GPAT and LPAAT, DGAT is believed to catalyze the rate-limiting step in TAG synthesis and represents a particularly interesting target for manipulating not only TAG content but also the fatty acid composition of TAG. Substantial TAG improvements (up to over twofold increase) by overexpressing $D G A T$ genes have been achieved in many algae including C. reinhardtti $[188,289], N$. oceanica $[189,194-196]$, and P. tricornutum [99, 198, 199, 290, 291], T. pseudonana [292], Scenedesmus obliquus [293] and Neochloris oleoabundans [294]. Of the ten C. zofingiensis DGAT genes, $D G A T 1 A$ and DGTT5, which are up-regulated considerably by ND, possess strong activities towards a broad range of substrates for TAG synthesis [189]. Overexpression of these two genes in C. zofingiensis may have the potential to boost TAG synthesis and production. Moreover, MLDP, the major structural protein of LDs that has been shown to promote TAG synthesis in yeast and $C$. reinhardtii via overexpression [40], is also a candidate target for TAG improvement in C. zofingiensis.

It is worth noting that TAG level in algae depends on not only biosynthesis but also catabolism. Protecting TAG against degradation represents another option to promote algal TAG level. Several TAG lipases from algae have been characterized and suppression of these lipase genes has proven to successfully enhance TAG content [207, 209, 210, 295, 296]. In C. zofingiensis, SDP1 and another putative TAG lipase encoded by Cz02g29090 are believed to participate in TAG degradation; suppression of them via knockdown or knockout should be beneficial to TAG accumulation. Moreover, the fatty acids released from TAG can enter peroxisomes and undergo degradation via the fatty acid $\beta$-oxidation process [159]. Inactivation of this process hinders TAG degradation and thus can increase TAG content, which has been achieved in $C$. reinhardtii by inactivating an $A O X$ gene [160]. This should also be applicable to C. zofingiensis via suppressing $A O X$ genes or $L A C S 5$ that encodes a peroxisomal enzyme response for converting free fatty acids to acyl-CoAs ready for downstream oxidation.

\section{Metabolic engineering for astaxanthin improvement}

In C. zofingiensis, the MEP pathway, not stimulated under astaxanthin inducing conditions, is likely a limiting factor for astaxanthin synthesis. Therefore, overexpression of $D X S, D X R$, and $H D R$, which are considered as key genes involved in controlling carbon flux of the MEP pathway [297-299], may provide sufficient precursors IPP/ DMAPP and push them to carotenoids for synthesizing more astaxanthin. Manipulation of the pathway that converts IPP/DMAPP to lycopene via overexpressing involved genes (e.g., PSY, PDS, ZDS) may also have the potential to enhance astaxanthin synthesis, as it can pull IPP/DMAPP away from sterols and other isoprenoids to carotenoids. In support of this, heterologous expression of C. zofingiensis PSY gene in C. reinhardtii resulted in enhanced carotenoid synthesis [239]. In addition, overexpression of PDS in C. zofingiensis enabled the alga to produce more total carotenoids as well as astaxanthin [34].

$\mathrm{LCYb}$ and LCYe compete for lycopene and control the carotenoid flux between $\beta$-carotene and $\alpha$-carotene derivatives and thus are promising engineering targets. Overexpression of $L C Y b$ may pull more carotenoid flux to $\beta$-carotene for downstream astaxanthin synthesis. On the other hand, suppressing LCYe can impair biosynthesis of lutein and its keto derivative keto-lutein, particularly the latter that accumulates under astaxnathin inducing conditions $[19,41]$, and thus has the potential to shunt the carotenoid flux from the $\alpha$-carotene branching route to the $\beta$-carotene branching route. Considering the low efficiency of $C$. zofingiensis BKT in ketolating zeaxanthin to astaxanthin and failure of $\mathrm{CHYb}$ in hydroxylating canthaxanthin to astaxanthin [14], introduction of a BKT converting zeaxanthin to astaxanthin efficiently and a CHYb with strong activity in converting canthaxanthin to astaxanthin is essential for minimizing the buildup of intermediates and pulling carotenoid flux to the end product astaxanthin, and represents a promising engineering strategy to increase astaxanthin content as well as purity. The truncated C. reinhardtii BKT and H. pluvialis $\mathrm{CHYb}$ are such enzyme pair for maximizing astaxanthin production from $\beta$-carotene, which have been evidenced in vascular plants [253, 270]. Overexpressing a gene responsible for astaxanthin esterification also has the potential to boost astaxanthin production, because it can on the one hand sequester free astaxanthin thereby releasing the product feedback inhibition on astaxanthin biosynthesis and on the other hand protect astaxanthin against degradation since esterified astaxanthin is more stable than free astaxanthin. Of course, such a gene needs 
to be characterized first. Furthermore, the carotenoids $\beta$-carotene and zeaxanthin that are synthesized in the chloroplast have to be exported out for astaxanthin biosynthesis. Therefore, promoting transport of carotenoid precursors across the chloroplast membranes is beneficial to astaxanthin synthesis.

\section{Metabolic engineering for both TAG and astaxanthin improvements}

The biosynthesis of TAG and astaxanthin each involves multiple coordinated steps. While manipulating a single gene can hardly obtain satisfactory increase of target products, multigene engineering is not easy to achieve in algae. Transcriptional factors (TFs) are a group of regulators that bind with certain upstream elements of their target genes and control their transcriptional expression. It has been reported in algae that TF manipulation is able to up-regulate multiple genes involved in lipid metabolism simultaneously and boost TAG accumulation [300303]. In C. zofingiensis, there are several TFs of MYB (Cz10g24240 and Cz06g23090), bHLH (Cz03g20070 and UNPLg00160) and bZIP (Cz15g21170 and UNPLg00449) that are predicted to regulate both lipid metabolism for TAG synthesis and carotenogenesis for astaxanthin synthesis [37, 41]. Overexpression of these TF genes may have the potential to achieve TAG and astaxanthin improvements concurrently in C. zofingiensis. Overexpressing the genes involved in NADPH production is also a possible direction, as both biosynthetic pathways need NADPH as the reductant. Glucose-6-phosphate 1-dehydrogenase (G6PD), 6-phosphogluconate dehydrogenase (6PGD) and malic enzyme (ME) are such targets of engineering in C. zofingiensis. Moreover, blocking the carbon competing pathways, e.g., starch biosynthesis via suppression of ADP-glucose pyrophosphorylase, may reroute the carbon flux to lipids and carotenoids leading to enhanced accumulation of both TAG and astaxanthin.

\section{Conclusions}

Despite substantial progresses achieved in the exploration of algal lipids for biodiesel production, the cost remains high and, therefore, restricts realization of commercial production of algae-derived biodiesel. Integrated production of algal lipids with value-added products represents one of the most promising strategies to improve the production economics of algal biodiesel. C. zofingiensis, able to grow robustly and achieve high biomass density under multiple trophic conditions, synthesizes TAG, the most energy-dense lipid ideal for making biodiesel, as well as astaxanthin, a high-value keto-carotenoid with broad applications. The simultaneous accumulation of TAG and astaxanthin allows integrated production of these two compounds by C. zofingiensis and thus has the potential to bring down the production cost of algal biodiesel. Since the release of chromosome-level whole genome sequence, many efforts have been made to better understand the pathways and regulation of TAG and astaxanthin biosynthesis in $C$. zofingiensis, which reveal distinctive features of this alga and help identify numerous gene targets for future engineering toward further improvements in TAG and/or astaxanthin levels. This has to rely on the establishment of more sophisticated genetic tools to allow easy and stable transformation, gene manipulation and genome editing of C. zofingiensis. Moreover, future directions also lie in development of next-generation culture systems for sustainable and cost-effective production of TAG-and-astaxanthin-rich biomass, and exploration of the state-of-art downstream processes for integrated production of TAG and astaxanthin. Breakthroughs occurring in these fields will greatly expand the production capacity and improve the production economics of C. zofingiensis.

\section{Abbreviations}

AACT: Acetoacetyl-CoA thiolase; AAT: Long-chain-alcohol O-fatty-acyltransferase; ACCase: Acetyl-CoA carboxylase; AdoMet: S-Adenosylmethionine; AOX: Acyl-CoA oxidase; BAT: Betaine lipid synthase; BKT: Beta-carotenoid ketolase; CDP-ME: 4-Diphosphocytidyl-2-C-methylerythritol; CDP-MEP: 4-Diphosphocytidyl-2-C-methyl-D-erythritol 2-phosphate; CDS: Phosphatidate cytidylyltransferase; CCT: Choline-phosphate cytidylyltransferase; CHK: Choline kinase; Cho: Choline; CHYb: Beta-carotenoid hydroxylase; CMK: 4-Diphosphocytidyl-2-C-methyl-D-erythritol kinase; CMS: 2-C-methyl-D-erythritol 4-phosphate cytidylyltransferase; CRTISO: Carotenoid isomerase; CYP97A: Cytochrome P450 beta hydroxylase; CYP97C: Cytochrome P450 epsilon hydroxylase; DAG: Diacylglycerol; DGAT: Diacylglycerol acyltransferase; DGD: Digalactosyldiacylglycerol synthase; DGDG: Digalactosyl diacylglycerol; DMAPP: Dimethylallyl pyrophosphate; DXR: 1-Deoxy-D-xylulose 5-phosphate reductoisomerase; DXP: 1-Deoxy-D-xylulose 5-phosphate; DXS: 1-Deoxy-D-xylulose 5-phosphate synthase; ECH: Enoyl-CoA hydratase; ECT: CDP-Ethanolamine synthase; ENR: Enoyl-ACP reductase; EPT/CPT: Ethanolaminephosphotransferase/ cholinephosphotransferase; Eth: Ethanolamine; ETK: Ethanolamine kinase; GALE: UDP-galactose 4-epimerase; FAD: Fatty acid desaturase; FAT: Acyl-ACP thioesterase; FPP: Farnesyl diphosphate; FPPS: Farnesyl diphosphate synthase; GAP: Glyceraldehyde 3-phosphate; GGPP: Geranylgeranyl diphosphate; GGPPS: Geranylgeranyl diphosphate synthase; G3P: Glycerol-3-phosphate; GPAT: Glycerol-3-phosphate acyltransferase; GPP: Geranyl diphosphate; GPPS: Geranyl diphosphate synthase; HCS: Hydroxymethylglutaryl-CoA synthase; HAD: 3-Ketoacyl-ACP dehydratase; HCD: 3-Hydroxyacyl-CoA dehydrogenase; HDR: 4-Hydroxy-3-methylbut-2-en-1-yl diphosphate reductase; HDS: 4-Hydroxy3-methylbut-2-en-1-yl diphosphate synthase; HGM-CoA: 3-Hydroxy-3-methylglutaryl-CoA; HMB-PP: (E)-4-Hydroxy-3-methyl-but-2-enyl pyrophosphate; IPP: Isopentenyl pyrophosphate; IPPI: Isopentenyl-diphosphate deltaisomerase; KAR: 3-Ketoacyl-ACP reductase; KAS: 3-Ketoacyl-ACP synthase; KATO: 3-Ketoacyl-COA thiolase; LACS: Long-chain acyl-CoA synthetase; LCYb: Lycopene beta cyclase; LCYe: Lycopene epsilon cyclase; LD: Lipid droplet; LPA: Lysophosphatidic acid; LPAAT: Lysophosphatidic acid acyltransferase; MCS: 2-C-methyl-D-erythritol 2,4-cyclodiphosphate synthase; MCT: MalonylCoA:acyl carrier protein transacylase; Met: Methionine; MECPP: 2-C-methylD-erythritol 2,4-cyclodiphosphate; MEP: 2-C-methylerythritol 4-phosphate; MIPS: Myo-inositol-1-phosphate synthase; MGD: Monogalactosyldiacylglycerol synthase; MGDG: Monogalactosyl diacylglycerol; MLDP: Major lipid droplet protein; NXS: Neoxanthin synthase; PA: Phosphatidic acid; PAP: Phosphatidate phosphatase; PC: Phosphatidylcholine; PDAT: Phospholipid:diacylglycerol acyltransferase; PDS: Phytoene desaturase; PE: Phosphatidylethanolamine; PEAMT: Phosphoethanolamine methyltransferase; PG: Phosphatidylglycerol; PGP: Phosphatidylglycerophosphatase; PGPS: Phosphatidylglycerophosphate 
synthase; PI: Phosphatidylinositol; PIS: Phosphatidylinositol synthase; PGD1: Plastid Galactoglycerolipid Degradation 1; PSY: Phytoene synthase; SAD: Stearoyl-ACP desaturase; SAS: S-adenosylmethionine synthase; SQDG: Sulfoquinovosyl diacylglycerol; SDP1: Sugar-Dependent1 TAG lipase; TAG :Triacyglycerol; UGPase: UDP-glucose pyrophosphorylase; VDE: Violaxanthin de-epoxidase; ZDS: Zeta-carotene desaturase; ZEP: Zeaxanthin epoxidase; ZISO: Zeta-carotene isomerase.

\section{Acknowledgements}

Not applicable.

\section{Authors' contributions}

$J L$ conceived the frame of the manuscript. $Y Z$ and $Y Y$ drafted the manuscript. $J \mathrm{~L}$ reviewed and edited the manuscript. All authors read and approved the final manuscript.

\section{Funding}

This work is partially supported by grants from the National Natural Science Foundation of China (32072183) and the National Key R\&D Program of China (2018YFA0902500).

\section{Availability of data and materials}

All data generated or analyzed during this study are included in this published article.

\section{Declarations}

\section{Ethics approval and consent to participate}

Not applicable.

\section{Consent for publication}

All authors approved the manuscript.

\section{Competing interests}

The authors declare that they have no competing interests.

Received: 12 March 2021 Accepted: 7 May 2021

Published online: 15 May 2021

\section{References}

1. Zhou W, Wang J, Chen P, Ji C, Kang Q, Lu B, et al. Bio-mitigation of carbon dioxide using microalgal systems: advances and perspectives. Renew Sust Energ Rev. 2017;76:1163-75.

2. Chisti Y. Biodiesel from microalgae. Biotechnol Adv. 2007;25:294-306.

3. Hu Q, Sommerfeld M, Jarvis E, Ghirardi M, Posewitz M, Seibert M, et al. Microalgal triacylglycerols as feedstocks for biofuel production: perspectives and advances. Plant J. 2008;54:621-39.

4. Wijffels RH, Barbosa MJ. An outlook on microalgal biofuels. Science. 2010;329:796-9.

5. Lenka SK, Carbonaro N, Park R, Miller SM, Thorpe I, Li Y. Current advances in molecular, biochemical, and computational modeling analysis of microalgal triacylglycerol biosynthesis. Biotechnol Adv. 2016;34:1046-63.

6. Raheem A, Prinsen P, Vuppaladadiyam AK, Zhao M, Luque R. A review on sustainable microalgae based biofuel and bioenergy production: recent developments. J Cleaner Prod. 2018;181:42-59.

7. Tang Y, Rosenberg JN, Bohutskyi P, Yu G, Betenbaugh MJ, Wang F. Microalgae as a feedstock for biofuel precursors and value-added products: green fuels and golden opportunities. BioResources. 2016;11:2850-85.

8. Liu J, Sun Z, Gerken H, Liu Z, Jiang Y, Chen F. Chlorella zofingiensis as an alternative microalgal producer of astaxanthin: biology and industrial potential. Mar Drugs. 2014;12:3487-515.

9. Lupette J, Benning C. Human health benefits of very-long-chain polyunsaturated fatty acids from microalgae. Biochimie. 2020;178:15-25.

10. Ambati R, Phang S-M, Ravi S, Aswathanarayana R. Astaxanthin: sources, extraction, stability, biological activities and its commercial applications-A review. Mar Drugs. 2014;12:128-52.
11. Dose J, Matsugo S, Yokokawa H, Koshida Y, Okazaki S, Seidel U, et al. Free radical scavenging and cellular antioxidant properties of astaxanthin. Int J Mol Sci. 2016;17:103

12. Galasso C, Orefice I, Pellone P, Cirino P, Miele R, lanora A, et al. On the neuroprotective role of astaxanthin: new perspectives? Mar drugs. 2018;16:247.

13. Liu J, Mao X, Zhou W, Guarnieri MT. Simultaneous production of triacylglycerol and high-value carotenoids by the astaxanthin-producing oleaginous green microalga Chlorella zofingiensis. Bioresour Technol. 2016;214:319-27.

14. Zhang Y, Ye Y, Ding W, Mao X, Li Y, Gerken H, et al. Astaxanthin is ketolated from zeaxanthin independent of fatty acid synthesis in Chromochloris zofingiensis. Plant Physiol. 2020;183:883-97.

15. Chen G, Wang B, Han D, Sommerfeld M, Lu Y, Chen F, et al. Molecular mechanisms of the coordination between astaxanthin and fatty acid biosynthesis in Haematococcus pluvialis (Chlorophyceae). Plant J. 2015;81:95-107.

16. Zhekisheva M, Zarka A, Khozin-Goldberg I, Cohen Z, Boussiba S. Inhibition of astaxanthin synthesis under high irradiance does not abolish triacylglycerol accumulation in the green alga Haematococcus pluvialis (Chlorophyceae). J Phycol. 2005;41:819-26.

17. Mao X, Wu T, Sun D, Zhang Z, Chen F. Differential responses of the green microalga Chlorella zofingiensis to the starvation of various nutrients for oil and astaxanthin production. Bioresour Technol. 2018;249:791-8.

18. Mao $X$, Zhang Y, Wang $X$, Liu J. Novel insights into salinity-induced lipogenesis and carotenogenesis in the oleaginous astaxanthin-producing alga Chromochloris zofingiensis: a multi-omics study. Biotechnol Biofuels. 2020;13:73.

19. Mulders KJM, Janssen JH, Martens DE, Wijffels RH, Lamers PP. Effect of biomass concentration on secondary carotenoids and triacylglycerol (TAG) accumulation in nitrogen-depleted Chlorella zofingiensis. Algal Res. 2014;6:8-16.

20. Mulders KM, Lamers P, Wijfels R, Martens D. Dynamics of biomass composition and growth during recovery of nitrogen-starved Chromochloris zofingiensis. Appl Microbiol Biotechnol. 2015;99:1873-84.

21. Chen JH, Liu L, Wei D. Enhanced production of astaxanthin by Chromochloris zofingiensis in a microplate-based culture system under high light irradiation. Bioresour Technol. 2017;245:518-29.

22. Del Campo JA, Rodriguez H, Moreno J, Vargas MA, Rivas J, Guerrero MG. Accumulation of astaxanthin and lutein in Chlorella zofingiensis (Chlorophyta). Appl Microbiol Biotechnol. 2004;64:848-54.

23. Ip PF, Chen F. Production of astaxanthin by the green microalga Chlorella zofingiensis in the dark. Process Biochem. 2005;40:733-8.

24. Ip PF, Wong KH, Chen F. Enhanced production of astaxanthin by the green microalga Chlorella zofingiensis in mixotrophic culture. Process Biochem. 2004;39:1761-6.

25. Liu J, Sun Z, Zhong Y, Gerken H, Huang J, Chen F. Utilization of cane molasses towards cost-saving astaxanthin production by a Chlorella zofingiensis mutant. J Appl Phycol. 2013;25:1447-56.

26. Sun N, Wang Y, Li Y-T, Huang J-C, Chen F. Sugar-based growth, astaxanthin accumulation and carotenogenic transcription of heterotrophic Chlorella zofingiensis (Chlorophyta). Process Biochem. 2008:43:1288-92.

27. Sun Z, Zhang Y, Sun L-p, Liu J. Light elicits astaxanthin biosynthesis and accumulation in the fermented ultrahigh-density Chlorella zofingiensis. J Agri Food Chem. 2019;67:5579-5586.

28. Sun Z, Zhou Z-g, Gerken H, Chen F, Liu J. Screening and characterization of oleaginous Chlorella strains and exploration of photoautotrophic Chlorella protothecoides for oil production. Bioresour Technol. 2015;184:53-62.

29. Chen J-h, Wei D, Lim P-E. Enhanced coproduction of astaxanthin and lipids by the green microalga Chromochloris zofingiensis: Selected phytohormones as positive stimulators. Bioresour Technol. 2020;295:122242

30. Liu J, Huang J, Fan KW, Jiang Y, Zhong Y, Sun Z, et al. Production potential of Chlorella zofingienesis as a feedstock for biodiesel. Bioresour Technol. 2010;101:8658-63.

31. Liu J, Huang J, Jiang Y, Chen F. Molasses-based growth and production of oil and astaxanthin by Chlorella zofingiensis. Bioresour Technol. 2012;107:393-8. 
32. Kou Y, Liu M, Sun P, Dong Z, Liu J. High light boosts salinity stressinduced biosynthesis of astaxanthin and lipids in the green alga Chromochloris zofingiensis. Algal Res. 2020;50:101976.

33. Roth MS, Cokus SJ, Gallaher SD, Walter A, Lopez D, Erickson E, et al. Chromosome-level genome assembly and transcriptome of the green alga Chromochloris zofingiensis illuminates astaxanthin production. Proc Natl Aca Sci USA. 2017;114:4296-305.

34. Liu J, Sun Z, Gerken H, Huang J, Jiang Y, Chen F. Genetic engineering of the green alga Chlorella zofingiensis: a modified norflurazon-resistant phytoene desaturase gene as a dominant selectable marker. Appl Microbiol Biotechnol. 2014;98:5069-79.

35. Roth MS, Westcott DJ, Iwai M, Niyogi KK. Hexokinase is necessary for glucose-mediated photosynthesis repression and lipid accumulation in a green alga. Commun Biology. 2019;2:347.

36. Ye Y, Huang J-C. Defining the biosynthesis of ketocarotenoids in Chromochloris zofingiensis. Plant Diversity. 2020;42:61-6.

37. Liu J, Sun Z, Mao X, Gerken H, Wang X, Yang W. Multiomics analysis reveals distinct mechanism of oleaginousness in the emerging model alga Chromochloris zofingiensis. Plant J. 2019;98:1060-77.

38. Roth MS, Gallaher SD, Westcott DJ, Iwai M, Louie KB, Mueller M, et al. Regulation of oxygenic photosynthesis during trophic transitions in the green alga Chromochloris zofingiensis. Plant Cell. 2019;31:579-601.

39. Mao X, Lao Y, Sun H, Li X, Yu J, Chen F. Timeresolved transcriptome analysis during transitions of sulfur nutritional status provides insight into triacylglycerol (TAG) and astaxanthin accumulation in the green alga Chromochloris zofingiensis. Biotechnol Biofuels. 2020;13:128.

40. Wang $X$, Wei H, Mao X, Liu J. Proteomics analysis of lipid droplets from the oleaginous alga Chromochloris zofingiensis reveals novel proteins for lipid metabolism. Genomics Proteomics Bioinformatics. 2019;17:260-72.

41. Zhang Y, Shi M, Mao X, Kou Y, Liu J. Time-resolved carotenoid profiling and transcriptomic analysis reveal mechanism of carotenogenesis for astaxanthin synthesis in the oleaginous green alga Chromochloris zofingiensis. Biotechnol Biofuels. 2019;12:287.

42. Dönz OC. Chlorella zofingiensis, eine neue Bodenalge. Bericht der Schweizerischen Botanischen Gesellschaft. 1934:43:127-31.

43. Hindák F. Taxonomic position of the chlorococcal alga Chlorella zofingiensis Dönz 1934 (Chlorophyceae). Algol Stud. 1982;40:13-23.

44. Kalina T, Punčochářová M. Taxonomy of the subfamily Scotiellocystoideae Fott 1976 (Chlorellaceae, Chlorophyceae). Algol Stud. 1987:45:473-521.

45. Huss VAR, Frank C, Hartmann EC, Hirmer M, Kloboucek A, Seidel BM, et al. Biochemical taxonomy and molecular phylogeny of the genus Chlorella sensu lato (Chlorophyta). J Phycol. 1999;35:587-98.

46. Hanagata N. Phylogeny of the subfamily Scotiellocystoideae (Chlorophyceae, Chlorophyta) and related taxa inferred from 18s ribosomal RNA gene sequence data. J Phycol. 1998;34:1049-54.

47. Krienitz L, Bock C, Dadheech PK, Pröschold T. Taxonomic reassessment of the genus Mychonastes (Chlorophyceae, Chlorophyta) including the description of eight new species. Phycologia. 2011;50:89-106.

48. Fučíková K, Lewis AL. Intersection of Chlorella, Muriella and Bracteacoccus: resurrecting the genus Chromochloris Kol et Chodat (Chlorophyceae, Chlorophyta). Fottea. 2012;12:83-93.

49. Azaman SNA, Nagao N, Yusoff FM, Tan SW, Yeap SK. A comparison of the morphological and biochemical characteristics of Chlorella sorokiniana and Chlorella zofingiensis cultured under photoautotrophic and mixotrophic conditions. PeerJ. 2017;5:e3473.

50. Koren I, Boussiba S, Khozin-Goldberg I, Zarka A. Chromochloris zofingiensis (Chlorophyceae) divides by consecutive multiple fission cell-cycle under batch and continuous cultivation. Biology. 2021;10:157.

51. Coleman AW. The nuclear cell cycle in Chlamydomonas (chlorophyceae). J Phycol. 1982;18:192-5.

52. Takeda H. Sugar composition of the cell wall and the taxonomy of chlorella (Chlorophyceae). J Phycol. 1991;27:224-32.

53. Takeda H. Chemical-composition of cell-walls as a taxonomical marker. J Plant Res. 1993;106:195-200.

54. Rise M, Cohen E, Vishkautsan M, Cojocaru M, Gottlieb HE, Arad SM. Accumulation of secondary carotenoids in Chlorella zofingiensis. J Plant Physiol. 1994;144:287-92.

55. Bar E, Rise M, Vishkautsan M, Arad S. Pigments and structural changes in Chlorella zofingiensis upon light and nitrogen stress. J Plant Physiol. 1995;146:527-34.
56. Lemoine Y, Schoefs B. Secondary ketocarotenoid astaxanthin biosynthesis in algae: a multifunctional response to stress. Photosynth Res. 2010:106:155-77.

57. Imaizumi Y, Nagao N, Yusoff FM, Taguchi S, Toda T. Estimation of optimum specific light intensity per cell on a high-cell-density continuous culture of Chlorella zofingiensis not limited by nutrients or $\mathrm{CO}_{2}$. Bioresour Technol. 2014;162:53-9.

58. Imaizumi Y, Nagao N, Yusoff FM, Kurosawa N, Kawasaki N, Toda T. Lumostatic operation controlled by the optimum light intensity per dry weight for the effective production of Chlorella zofingiensis in the high cell density continuous culture. Algal Res. 2016;20:110-7.

59. Bonente G, Passarini F, Cazzaniga S, Mancone C, Buia MC, Tripodi M, et al. The occurrence of the psbS gene product in Chlamydomonas reinhardtii and in other photosynthetic organisms and its correlation with energy quenching. Photochem Photobiol. 2008;84:1359-70.

60. Sudibyo H, Pradana YS, Samudra TT, Budiman A, Indarto, Suyono EA. Study of cultivation under different colors of light and growth kinetic study of Chlorella zofingiensis dönz for biofuel production. Energy Procedia. 2017;105:270-276.

61. Minyuk G, Dantsyuk N, Chelebieva E, Chubchikova I, Drobetskaya I, Solovchenko A. The effect of diverse nitrogen sources in the nutrient medium on the growth of the green microalgae Chromochloris zofingiensis in the batch culture. Mar Biol J. 2019;4:41-52.

62. Minyuk G, Sidorov R, Solovchenko A. Effect of nitrogen source on the growth, lipid, and valuable carotenoid production in the green microalga Chromochloris zofingiensis. J Appl Phycol. 2020;32:923-35.

63. Goldman JC, Brewer PG. Effect of nitrogen source and growth rate on phytoplankton-mediated changes in alkalinity. Limno Oceano. 1980;25:352-7.

64. Liu J, Sommerfeld M, Hu Q. Screening and characterization of Isochrysis strains and optimization of culture conditions for docosahexaenoic acid production. Appl Microbiol Biotechnol. 2013;97:4785-98.

65. Shi X-M, Zhang X-W, Chen F. Heterotrophic production of biomass and lutein by Chlorella protothecoides on various nitrogen sources. Enzy Microb Technol. 2000;27:312-8.

66. Hata N, Ogbonna JC, Hasegawa Y, Taroda H, Tanaka H. Production of astaxanthin by Haematococcus pluvialis in a sequential heterotrophicphotoautotrophic culture. J Appl Phycol. 2001;13:395-402.

67. Zaslavskaia LA, Lippmeier JC, Shih C, Ehrhardt D, Grossman AR, Apt KE. Trophic conversion of an obligate photoautotrophic organism through metabolic engineering. Science. 2001;292:2073.

68. Zhang Z, Huang JJ, Sun D, Lee Y, Chen F. Two-step cultivation for production of astaxanthin in Chlorella zofingiensis using a patented energy-free rotating floating photobioreactor (RFP). Bioresour Technol. 2017; $224: 515-22$

69. Zhang Z, Sun D, Wu T, Li Y, Lee Y, Liu J, et al. The synergistic energy and carbon metabolism under mixotrophic cultivation reveals the coordination between photosynthesis and aerobic respiration in Chlorella zofingiensis. Algal Res. 2017;25:109-16.

70. Sun H, Ren Y, Lao Y, Li X, Chen F. A novel fed-batch strategy enhances lipid and astaxanthin productivity without compromising biomass of Chromochloris zofingiensis. Bioresour Technol. 2020;308:123306.

71. Boyle NR, Page MD, Liu B, Blaby IK, Casero D, Kropat J, et al. Three acyltransferases and nitrogen-responsive regulator are implicated in nitrogen starvation-induced triacylglycerol accumulation in Chlamydomonas. J Biol Chem. 2012;287:15811-25.

72. Van Wagenen J, Miller TW, Hobbs S, Hook P, Crowe B, Huesemann M. Effects of light and temperature on fatty acid production in Nannochloropsis salina. Energies. 2012;5:731-40.

73. Jia J, Han D, Gerken HG, Li Y, Sommerfeld M, Hu Q, et al. Molecular mechanisms for photosynthetic carbon partitioning into storage neutral lipids in Nannochloropsis oceanica under nitrogen-depletion conditions. Algal Res. 2015;7:66-77.

74. Abida H, Dolch LJ, Mei C, Villanova V, Conte M, Block MA, et al. Membrane glycerolipid remodeling triggered by nitrogen and phosphorus starvation in Phaeodactylum tricornutum. Plant Physiol. 2015;167:118-36.

75. Xing G, Yuan H, Yang J, Li J, Gao Q, Li W, et al. Integrated analyses of transcriptome, proteome and fatty acid profilings of the oleaginous microalga Auxenochlorella protothecoides UTEX 2341 reveal differential 
reprogramming of fatty acid metabolism in response to low and high temperatures. Algal Res. 2018;33:16-27.

76. Pal D, Khozin-Goldberg I, Cohen Z, Boussiba S. The effect of light, salinity, and nitrogen availability on lipid production by Nannochloropsis sp. Appl Microbiol Biotechnol. 2011;90:1429-41.

77. Wang Y, Liu Z, Qin S. Effects of iron on fatty acid and astaxanthin accumulation in mixotrophic Chromochloris zofingiensis. Biotechnol Lett. 2013;35:351-7.

78. Zhu S, Huang W, Xu J, Wang Z, Xu J, Yuan Z. Metabolic changes of starch and lipid triggered by nitrogen starvation in the microalga Chlorella zofingiensis. Bioresour Technol. 2014;152:292-8.

79. Liu J, Huang J, Sun Z, Zhong Y, Jiang Y, Chen F. Differential lipid and fatty acid profiles of photoautotrophic and heterotrophic Chlorella zofingiensis: Assessment of algal oils for biodiesel production. Bioresour Technol. 2011;102:106-10.

80. Suyono EA, Listyarini A, Hardiyati A. Effect high salinity and red-blue light treatment on lipid content of the microalgae Chlorella zofingiensis Dönz as biodiesel source. Appl Mecha Mater. 2016;842:103-10.

81. Feng P, Deng Z, Hu Z, Fan L. Lipid accumulation and growth of Chlorella zofingiensis in flat plate photobioreactors outdoors. Bioresour Technol. 2011;102:10577-84.

82. Jiang X, Liu L, Chen J, Wei D. Effects of Xanthophyllomyces dendrorhous on cell growth, lipid, and astaxanthin production of Chromochloris zofingiensis by mixed culture strategy. J Appl Phycol. 2018;30:3009-15.

83. $\mathrm{Xu} \mathrm{H}$, Miao X, Wu Q. High quality biodiesel production from a microalga Chlorella protothecoides by heterotrophic growth in fermenters. J Biotechnol. 2006;126:499-507.

84. Cheng Y, Zhou W, Gao C, Lan K, Gao Y, Wu Q. Biodiesel production from Jerusalem artichoke (Helianthus Tuberosus L.) tuber by heterotrophic microalgae Chlorella protothecoides. J Chem Technol Biotechnol. 2009:84:777-81.

85. Yan D, Lu Y, Chen Y-F, Wu Q. Waste molasses alone displaces glucosebased medium for microalgal fermentation towards cost-saving biodiesel production. Bioresour Technol. 2011;102:6487-93.

86. Shi Y, Liu M, Ding W, Liu J. Novel insights into phosphorus deprivationboosted lipid synthesis in the marine alga Nannochloropsis oceanica without compromising biomass production. J Agri Food Chem. 2020:68:11488-502.

87. Mühlroth A, Winge $P$, El Assimi A, Jouhet J, Maréchal E, HohmannMarriott MF, et al. Mechanisms of phosphorus acquisition and lipid class remodeling under $\mathrm{P}$ limitation in a marine microalga. Plant Physiol. 2017:175:1543-59.

88. Orosa M, Valero JF, Herrero C, Abalde J. Comparison of the accumulation of astaxanthin in Haematococcus pluvialis and other green microalgae under $\mathrm{N}$-starvation and high light conditions. Biotechnol Lett. 2001;23:1079-85.

89. Shi M, Wei H, Chen Q, Wang X, Zhou W, Liu J. Exploring an isolate of the oleaginous alga Micractinium inermum for lipid production: molecular characterization and physiochemical analysis under multiple growth conditions. J Appl Phycol. 2019;31:1035-46.

90. Hirai K, Hayashi T, Hasegawa Y, Sato A, Tsuzuki M, Sato N. Hyperosmosis and its combination with nutrient-limitation are novel environmental stressors for induction of triacylglycerol accumulation in cells of Chlorella kessleri. Sci Rep. 2016;6:25825.

91. Kim B-H, Ramanan R, Kang Z, Cho D-H, Oh H-M, Kim H-S. Chlorella sorokiniana HS1, a novel freshwater green algal strain, grows and hyperaccumulates lipid droplets in seawater salinity. Biomass Bioenerg. 2016;85:300-5.

92. Xia L, Rong J, Yang $\mathrm{H}, \mathrm{He} \mathrm{Q}$, Zhang $\mathrm{D}, \mathrm{Hu} \mathrm{C}$. $\mathrm{NaCl}$ as an effective inducer for lipid accumulation in freshwater microalgae Desmodesmus abundans. Bioresour Technol. 2014;161:402-9.

93. Ferreira GF, Ríos Pinto LF, Maciel Filho R, Fregolente LV. A review on lipid production from microalgae: association between cultivation using waste streams and fatty acid profiles. Renew Sustain Energ Rev. 2019;109:448-66.

94. Griffiths M, Harrison S. Lipid productivity as a key characteristic for choosing algal species for biodiesel production. J Appli Phycol. 2009;21:493-507.

95. Mata TM, Martins AA, Caetano NS. Microalgae for biodiesel production and other applications: a review. Renew Sustain Energ Rev. 2010;14:217-32.
96. Breuer G, Lamers PP, Martens DE, Draaisma RB, Wijffels RH. The impact of nitrogen starvation on the dynamics of triacylglycerol accumulation in nine microalgae strains. Bioresour Technol. 2012;124:217-26.

97. Guccione A, Biondi N, Sampietro G, Rodolfi L, Bassi N, Tredici M. Chlorella for protein and biofuels: from strain selection to outdoor cultivation in a Green Wall Panel photobioreactor. Biotechnol Biofuels. 2014;7:84.

98. Ma X, Chen T, Yang B, Liu J, Chen F. Lipid production from Nannochloropsis. Mar Drugs. 2016;14:61.

99. Haslam RP, Hamilton ML, Economou CK, Smith R, Hassall KL, Napier $J A$, et al. Overexpression of an endogenous type 2 diacylglycerol acyltransferase in the marine diatom Phaeodactylum tricornutum enhances lipid production and omega-3 long-chain polyunsaturated fatty acid content. Biotechnol Biofuels. 2020;13:87.

100. Knothe G. Improving biodiesel fuel properties by modifying fatty ester composition. Energ Environ Sci. 2009;2:759-66.

101. Knothe G. "Designer" biodiesel: optimizing fatty ester composition to improve fuel properties. Energ Fuel. 2008;22:1358-64.

102. Jin E, Polle JEW, Lee HK, Hyun SM, Chang M. Xanthophylls in microalgae: from biosynthesis to biotechnological mass production and application. J Microbiol Biotechnol. 2003;13:165-74.

103. Harker M, Young AJ. Inhibition of astaxanthin synthesis in the green alga Haematococcus pluvialis. Euro J Phycol. 1995;30:179-87.

104. Orosa M, Torres E, Fidalgo P, Abalde J. Production and analysis of secondary carotenoids in green algae. J Appl Phycol. 2000;12:553-6.

105. Toyoshima H, Takaichi S, Kawasaki S. Water-soluble astaxanthin-binding protein (AstaP) from Coelastrella astaxanthina Ki-4 (Scenedesmaceae) involving in photo-oxidative stress tolerance. Algal Res. 2020;50:101988.

106. Qin S, Liu G-X, Hu Z-Y. The accumulation and metabolism of astaxanthin in Scenedesmus obliquus (Chlorophyceae). Process Biochem. 2008:43:795-802.

107. Mulders KM, Weesepoel Y, Bodenes P, Lamers P, Vincken J-P, Martens $D$, et al. Nitrogen-depleted Chlorella zofingiensis produces astaxanthin, ketolutein and their fatty acid esters: a carotenoid metabolism study. J Appl Phycol. 2015;27:125-40.

108. Grunewald K, Hirschberg J, Hagen C. Ketocarotenoid biosynthesis outside of plastids in the unicellular green alga Haematococcus pluvialis. J Biol Chem. 2001:276:6023-9.

109. Grunewald K, Hagen C. Beta-carotene is the intermediate exported from the chloroplast during accumulation of secondary carotenoids in Haematococcus pluvialis. J Appl Phycol. 2001;13:89-93.

110. Lorenz RT, Cysewski GR. Commercial potential for Haematococcus microalgae as a natural source of astaxanthin. Trends Biotechnol. 2000;18:160-7.

111. Han D, LiY, Hu Q. Astaxanthin in microalgae: pathways, functions and biotechnological implications. Algae. 2013.

112. Del Campo JA, Moreno J, Rodríguez H, Angeles Vargas M, Rivas Jn, Guerrero MG. Carotenoid content of chlorophycean microalgae: factors determining lutein accumulation in Muriellopsis sp. (Chlorophyta). J Biotechnol. 2000;76:51-59.

113. Ip PF, Chen F. Employment of reactive oxygen species to enhance astaxanthin formation in Chlorella zofingiensis in heterotrophic culture. Process Biochem. 2005:40:3491-6.

114. Ip PF, Chen F. Peroxynitrite and nitryl chloride enhance astaxanthin production by the green microalga Chlorella zofingiensis in heterotrophic culture. Process Biochem. 2005;40:3595-9.

115. Zhao Y, Wang H-P, Han B, Yu X. Coupling of abiotic stresses and phytohormones for the production of lipids and high-value by-products by microalgae: a review. Bioresour Technol. 2019;274:549-56.

116. Wan M, Zhang Z, Wang J, Huang J, Fan J, Yu A, et al. Sequential heterotrophy-dilution-photoinduction cultivation of Haematococcus pluvialis for efficient production of astaxanthin. Bioresour Technol. 2015;198:557-63.

117. Ranjbar R, Inoue R, Shiraishi H, Katsuda T, Katoh S. High efficiency production of astaxanthin by autotrophic cultivation of Haematococcus pluvialis in a bubble column photobioreactor. Biochem Eng J. 2008:39:575-80.

118. Zhang XW, Gong XD, Chen F. Dynamics and stability analysis of the growth and astaxanthin production system of Haematococcus pluvialis. J Ind Microbiol Biotechnol. 1999;23:133-7. 
119. López MCG-M, Sánchez EDR, López JLC, Fernández FGA, Sevilla JMF, Rivas J, et al. Comparative analysis of the outdoor culture of Haematococcus pluvialis in tubular and bubble column photobioreactors. J Biotechnol. 2006;123:329-342.

120. Wen Z, Liu Z, Hou Y, Liu C, Gao F, Zheng Y, et al. Ethanol induced astaxanthin accumulation and transcriptional expression of carotenogenic genes in Haematococcus pluvialis. Enzy Microbial Technol. 2015;78:10-7.

121. Sun H, Kong Q, Geng Z, Duan L, Yang M, Guan B. Enhancement of cell biomass and cell activity of astaxanthin-rich Haematococcus pluvialis. Bioresour Technol. 2015;186:67-73.

122. Kopecky J, Schoefs B, Loest K, Stys D, Pulz O. Microalgae as a source for secondary carotenoid production: a screening study. Algol Stud. 2000:98:153-68.

123. Sun Z, Liu J, Zeng $X$, Huangfu J, Jiang Y, Wang M, et al. Astaxanthin is responsible for antiglycoxidative properties of microalga Chlorella zofingiensis. Food Chem. 2011;126:1629-35.

124. Kobayashi M, Sakamoto Y. Singlet oxygen quenching ability of astaxanthin esters from the green alga Haematococcus pluvialis. Biotechnol Lett. 1999;21:265-9.

125. Postma PR, Miron TL, Olivieri G, Barbosa MJ, Wijffels RH, Eppink MHM. Mild disintegration of the green microalgae Chlorella vulgaris using bead milling. Bioresour Technol. 2015;184:297-304.

126. Zheng H, Yin J, Gao Z, Huang H, Ji X, Dou C. Disruption of Chlorella vulgaris cells for the release of biodiesel-producing lipids: a comparison of grinding, ultrasonication, bead milling, enzymatic lysis, and microwaves. Appl Biochem Biotechnol. 2011;164:1215-24.

127. Greenly JM, Tester JW. Ultrasonic cavitation for disruption of microalgae. Bioresour Technol. 2015;184:276-9.

128. Xie Y, Ho SH, Chen CNN, Chen CY, Jing K, Ng IS, et al. Disruption of thermo-tolerant Desmodesmus sp. F51 in high pressure homogenization as a prelude to carotenoids extraction. Biochem Eng J. 2016;109:243-51.

129. Topare N, Raut S, Renge V, Khedkar S, Chauhan YP, Bhagat S. Extraction of oil from algae by solvent extraction and oil expeller method. Int J Chem Sci. 2011;9:1746-50.

130. Thoisen C, Hansen BW, Nielsen SL. A simple and fast method for extraction and quantification of cryptophyte phycoerythrin. MethodsX. 2017:4:209-13.

131. González-González LM, Astals S, Pratt S, Jensen PD, Schenk PM. Impact of osmotic shock pre-treatment on microalgae lipid extraction and subsequent methane production. Bioresour Technol Rep. 2019;7:100214.

132. Balasubramanian S, Allen JD, Kanitkar A, Boldor D. Oil extraction from Scenedesmus obliquus using a continuous microwave systemdesign, optimization, and quality characterization. Bioresour Technol. 2011;102:3396-403

133. Gerken HG, Donohoe B, Knoshaug EP. Enzymatic cell wall degradation of Chlorella vulgaris and other microalgae for biofuels production. Planta. 2013;237:239-53.

134. Gorgich M, Mata TM, Martins AA, Branco-Vieira M, Caetano NS. Comparison of different lipid extraction procedures applied to three microalgal species. Energ Rep. 2020;6:477-82.

135. Lee SJ, Yoon B-D, Oh H-M. Rapid method for the determination of lipid from the green alga Botryococcus braunii. Biotechnol Tech. 1998;12:553-6.

136. Ryckebosch E, Muylaert K, Foubert I. Optimization of an analytical procedure for extraction of lipids from microalgae. J Am Oil Chem Soc. 2012;89:189-98.

137. Kim Y-H, Choi Y-K, Park J, Lee S, Yang Y-H, Kim HJ, et al. lonic liquidmediated extraction of lipids from algal biomass. Bioresour Technol. 2012;109:312-5.

138. Obeid S, Beaufils N, Camy S, Takache H, Ismail A, Pontalier P-Y. Supercritical carbon dioxide extraction and fractionation of lipids from freezedried microalgae Nannochloropsis oculata and Chlorella vulgaris. Algal Res. 2018;34:49-56.

139. Orr VCA, Plechkova NV, Seddon KR, Rehmann L. Disruption and wet extraction of the microalgae Chlorella vulgaris using room-temperature ionic liquids. ACS Sustain Chem Eng. 2016;4:591-600.

140. Patil PD, Dandamudi KPR, Wang J, Deng Q, Deng S. Extraction of bio-oils from algae with supercritical carbon dioxide and co-solvents. J Supercrit Fluids. 2018;135:60-8.
141. Li-Beisson Y, Beisson F, Riekhof W. Metabolism of acyl-lipids in Chlamydomonas reinhardtii. Plant J. 2015;82:504-22.

142. Terashima M. Chlamydomonas: triacylglycerol accumulation. In: Hippler M, editor. Chlamydomonas: biotechnology and biomedicine. Cham: Springer International Publishing; 2017. p. 193-217.

143. Merchant SS, Kropat J, Liu B, Shaw J, Warakanont J. TAG, You're it! Chlamydomonas as a reference organism for understanding algal triacylglycerol accumulation. Curr Opin Biotechnol. 2012;23:352-63.

144. Rawsthorne S. Carbon flux and fatty acid synthesis in plants. Prog Lipid Res. 2002;41:182-96.

145. Ohlrogge JB, Jaworski JG. Regulation of fatty acid synthesis. Annu Rev Plant Physiol Plant Mol Biol. 1997;48:109-36.

146. Li N, Gügel IL, Giavalisco P, Zeisler V, Schreiber L, Soll J, et al. FAX1, a novel membrane protein mediating plastid fatty acid export. PLoS Biol. 2015;13:e1002053.

147. Li N, Zhang Y, Meng H, Li S, Wang S, Xiao Z, et al. Characterization of Fatty Acid EXporters involved in fatty acid transport for oil accumulation in the green alga Chlamydomonas reinhardtii. Biotechnol Biofuels. 2019;12:14.

148. Takemura T, Imamura S, Tanaka K. Identification of a chloroplast fatty acid exporter protein, CmFAX1, and triacylglycerol accumulation by its overexpression in the unicellular red alga Cyanidioschyzon merolae. Algal Res. 2019;38:101396.

149. Shockey JM, Fulda MS, Browse JA. Arabidopsis contains nine long-chain acyl-coenzyme A synthetase genes that participate in fatty acid and glycerolipid metabolism. Plant Physiol. 2002;129:1710.

150. Jia B, Song Y, Wu M, Lin B, Xiao K, Hu Z, et al. Characterization of long-chain acyl-CoA synthetases which stimulate secretion of fatty acids in green algae Chlamydomonas reinhardtii. Biotechnol biofuels. 2016:9:184-184.

151. Wu T, Fu Y, Shi Y, Li Y, Kou Y, Mao X, et al. Functional characterization of long-chain acyl-CoA synthetase gene family from the oleaginous alga Chromochloris zofingiensis. J Agri Food Chem. 2020;68:4473-84.

152. Guo X, Jiang M, Wan X, Hu C, Gong Y. Identification and biochemical characterization of five long-chain acyl-coenzyme A synthetases from the diatom Phaeodactylum tricornutum. Plant Physiol Biochem. 2014;74:33-41.

153. Tonon T, Qing R, Harvey D, Li Y, Larson TR, Graham IA. Identification of a long-chain polyunsaturated fatty acid acyl-coenzyme A synthetase from the diatom Thalassiosira pseudonana. Plant Physiol. 2005;138:402.

154. Sobrado P, Lyle KS, Kaul SP, Turco MM, Arabshahi I, Marwah A, et al. Identification of the binding region of the [2Fe-2S] ferredoxin in stearoylacyl carrier protein desaturase: insight into the catalytic complex and mechanism of action. Biochem. 2006;45:4848-58.

155. Harwood JL. Recent advances in the biosynthesis of plant fatty acids. Biochim Biophysi Acta. 1996;1301:7-56.

156. Liu J, Sun Z, Zhong Y, Huang J, Hu Q, Chen F. Stearoyl-acyl carrier protein desaturase gene from the oleaginous microalga Chlorella zofingiensis: cloning, characterization and transcriptional analysis. Planta. 2012;236:1665-76.

157. Chi X, Zhang X, Guan X, Ding L, Li Y, Wang M, et al. Fatty acid biosynthesis in eukaryotic photosynthetic microalgae: Identification of a microsomal delta 12 desaturase in Chlamydomonas reinhardtii. J Microbiol. 2008;46:189-201.

158. Nguyen HM, Cuiné S, Beyly-Adriano A, Légeret B, Billon E, Auroy $P$ et al. The green microalga Chlamydomonas reinhardtii has a single $\omega-3$ fatty acid desaturase which localizes to the chloroplast and impacts both plastidic and extraplastidic membrane lipids. Plant Physiol. 2013;163:914-28.

159. Kong F, Romero IT, Warakanont J, Li-Beisson Y. Lipid catabolism in microalgae. New Phytol. 2018;218:1340-8.

160. Kong F, Liang Y, Legeret B, Beyly-Adriano A, Blangy S, Haslam RP, et al. Chlamydomonas carries out fatty acid beta-oxidation in ancestral peroxisomes using a bona fide acyl-CoA oxidase. Plant J. 2017;90:358-71.

161. Poirier Y, Antonenkov VD, Glumoff T, Hiltunen JK. Peroxisomal B-oxidation - a metabolic pathway with multiple functions. Biochim Biophys Acta Mol Cell Res. 2006;1763:1413-26.

162. Li-Beisson Y, Thelen JJ, Fedosejevs E, Harwood JL. The lipid biochemistry of eukaryotic algae. Prog Lipid Res. 2019;74:31-68. 
163. Shimojima M. Biosynthesis and functions of the plant sulfolipid. Prog Lipid Res. 2011;50:234-9.

164. Riekhof WR, Sears BB, Benning C. Annotation of genes involved in glycerolipid biosynthesis in Chlamydomonas reinhardtii: discovery of the betaine lipid synthase BTA1 ${ }_{C r}$. Eukaryot Cell. 2005;4:242-52.

165. Pineau B, Girard-Bascou J, Eberhard S, Choquet Y, Trémolières A, GérardHirne C, et al. A single mutation that causes phosphatidylglycerol deficiency impairs synthesis of photosystem II cores in Chlamydomonas reinhardtii. Eur J Biochem. 2004;271:329-38.

166. Sugimoto K, Midorikawa T, Tsuzuki M, Sato N. Upregulation of PG synthesis on sulfur-starvation for PS I in Chlamydomonas. Biochem Biophys Res Commun. 2008;369:660-5.

167. Gibellini F, Smith TK. The Kennedy pathway-De novo synthesis of phosphatidylethanolamine and phosphatidylcholine. IUBMB Life. 2010;62:414-28.

168. Sato N, Mori N, Hirashima T, Moriyama T. Diverse pathways of phosphatidylcholine biosynthesis in algae as estimated by labeling studies and genomic sequence analysis. Plant J. 2016;87:281-92.

169. Li X, Moellering ER, Liu B, Johnny C, Fedewa M, Sears BB, et al. A galactoglycerolipid lipase is required for triacylglycerol accumulation and survival following nitrogen deprivation in Chlamydomonas reinhardtii. Plant Cell. 2012;24:4670-86.

170. Du Z-Y, Lucker BF, Zienkiewicz K, Miller TE, Zienkiewicz A, Sears BB, et al. Galactoglycerolipid lipase PGD1 is involved in thylakoid membrane remodeling in response to adverse environmental conditions in Chlamydomonas. Plant Cell. 2018;30:447-65.

171. Yoon K, Han D, Li Y, Sommerfeld M, Hu Q. Phospholipid:diacylglycerol acyltransferase is a multifunctional enzyme involved in membrane lipid turnover and degradation while synthesizing triacylglycerol in the unicellular green microalga Chlamydomonas reinhardtii. Plant Cell. 2012;24:3708-24.

172. Liu X-Y, Ouyang L-L, Zhou Z-G. Phospholipid: diacylglycerol acyltransferase contributes to the conversion of membrane lipids into triacylglycerol in Myrmecia incisa during the nitrogen starvation stress. Sci rep. 2016;6:26610-26610.

173. Fan J, Yan C, Zhang X, Xu C. Dual role for phospholipid:diacylglycerol acyltransferase: enhancing fatty acid synthesis and diverting fatty acids from membrane lipids to triacylglycerol in Arabidopsis leaves. Plant Cell. 2013;25:3506-18.

174. Jayawardhane KN, Singer SD, Weselake RJ, Chen G. Plant sn-glycerol3-phosphate acyltransferases: biocatalysts involved in the biosynthesis of intracellular and extracellular lipids. Lipids. 2018;53:469-80.

175. Jaeger D, Winkler A, Mussgnug JH, Kalinowski J, Goesmann A, Kruse O. Time-resolved transcriptome analysis and lipid pathway reconstruction of the oleaginous green microalga Monoraphidium neglectum reveal a model for triacylglycerol and lipid hyperaccumulation. Biotechnol Biofuels. 2017;10:197.

176. Sun L-P, Ouyang L-L, Bao H, Liu J-G, Sun Z, Zhou Z-G. Comparison between two isoforms of glycerol-3-phosphate acyltransferase in microalga Myrmecia incisa: Subcellular localization and role in triacylglycerol synthesis. Algal Res. 2021;54:102172.

177. Li J, Han D, Wang D, Ning K, Jia J, Wei L, et al. Choreography of transcriptomes and lipidomes of Nannochloropsis reveals the mechanisms of oil synthesis in microalgae. Plant Cell. 2014;26:1645-65.

178. Levitan O, Dinamarca J, Zelzion E, Lun DS, Guerra LT, Kim MK, et al. Remodeling of intermediate metabolism in the diatom Phaeodactylum tricornutum under nitrogen stress. Proc Natl Acad Sci USA. 2015;112:412-7.

179. Fukuda S, Hirasawa E, Takemura T, Takahashi S, Chokshi K, Pancha I, et al. Accelerated triacylglycerol production without growth inhibition by overexpression of a glycerol-3-phosphate acyltransferase in the unicellular red alga Cyanidioschyzon merolae. Sci Rep. 2018;8:12410.

180. Wang X, Liu S-F, Li R-Y, Yang W-D, Liu J-S, Lin CSK, et al. TAG pathway engineering via GPAT2 concurrently potentiates abiotic stress tolerance and oleaginicity in Phaeodactylum tricornutum. Biotechnol Biofuels. 2020:13:160.

181. Yamaoka Y, Achard D, Jang S, Legeret B, Kamisuki S, Ko D, et al. Identification of a Chlamydomonas plastidial 2-lysophosphatidic acid acyltransferase and its use to engineer microalgae with increased oil content. Plant Biotechnol J. 2016;14:2158-67.
182. Kim Y, Terng EL, Riekhof WR, Cahoon EB, Cerutti H. Endoplasmic reticulum acyltransferase with prokaryotic substrate preference contributes to triacylglycerol assembly in Chlamydomonas. Proc Natl Acad Sci. 2018;115:1652-7.

183. Liu J, Han D, Yoon K, Hu Q, Li Y. Characterization of type 2 diacylglycerol acyltransferases in Chlamydomonas reinhardtii reveals their distinct substrate specificities and functions in triacylglycerol biosynthesis. Plant J. 2016;86:3-19.

184. Fan J, Andre C, Xu C. A chloroplast pathway for the de novo biosynthesis of triacylglycerol in Chlamydomonas reinhardtii. FEBS Lett. 2011;585:1985-91.

185. Deng X-d, Cai J-j, Fei X-w. Involvement of phosphatidate phosphatase in the biosynthesis of triacylglycerols in Chlamydomonas reinhardtii. J Zhejiang Univ Sci B. 2013;14:1121-31.

186. Xu Y, Caldo KMP, Pal-Nath D, Ozga J, Lemieux MJ, Weselake RJ, et al. Properties and biotechnological applications of acyl-coa:diacylglycerol acyltransferase and phospholipid:diacylglycerol acyltransferase from terrestrial plants and microalgae. Lipids. 2018;53:663-88.

187. Sanjaya, Miller R, Durrett TP, Kosma DK, Lydic TA, Muthan B, et al. Altered lipid composition and enhanced nutritional value of Arabidopsis leaves following introduction of an algal diacylglycerol acyltransferase 2. Plant Cell. 2013;25:677-693.

188. Iwai M, Ikeda K, Shimojima M, Ohta H. Enhancement of extraplastidic oil synthesis in Chlamydomonas reinhardtii using a type-2 diacylglycerol acyltransferase with a phosphorus starvation-inducible promoter. Plant Biotechnol J. 2014;12:808-19.

189. Mao X, Wu T, Kou Y, Shi Y, Zhang Y, Liu J. Characterization of type I and type II diacylglycerol acyltransferases from the emerging model alga Chlorella zofingiensis reveals their functional complementarity and engineering potential. Biotechnol Biofuels. 2019;12:28.

190. Xu Y, Falarz L, Chen G. Characterization of type-2 diacylglycerol acyltransferases in the green microalga Chromochloris zofingiensis. J Agri Food Chem. 2019:67:291-8.

191. Xu Y, Caldo KMP, Falarz L, Jayawardhane K, Chen G. Kinetic improvement of an algal diacylglycerol acyltransferase 1 via fusion with an acyl-CoA binding protein. Plant J. 2020;102:856-71.

192. Ma H, Wu X, Wei Z, Zhao L, Li Z, Liang Q, et al. Functional divergence of diacylglycerol acyltransferases in the unicellular green alga Haematococcus pluvialis. J Exp Bot. 2020;72:510-24.

193. Cui H, Zhao C, Xu W, Zhang H, Hang W, Zhu X, et al. Characterization of type-2 diacylglycerol acyltransferases in Haematococcus lacustris reveals their functions and engineering potential in triacylglycerol biosynthesis. BMC Plant Biol. 2021;21:20.

194. Wei H, Shi Y, Ma X, Pan Y, Hu H, Li Y, et al. A type-I diacylglycerol acyltransferase modulates triacylglycerol biosynthesis and fatty acid composition in the oleaginous microalga Nannochloropsis oceanica. Biotechnol Biofuels. 2017;10:174.

195. Xin Y, Lu Y, Lee Y-Y, Wei L, Jia J, Wang Q, et al. Producing designer oils in industrial microalgae by rational modulation of co-evolving type-2 diacylglycerol acyltransferases. Mol Plant. 2017;10:1523-39.

196. Zienkiewicz K, Zienkiewicz A, Poliner E, Du Z-Y, Vollheyde K, Herrfurth C, et al. Nannochloropsis, a rich source of diacylglycerol acyltransferases for engineering of triacylglycerol content in different hosts. Biotechnol Biofuels. 2017:10:8.

197. Guiheneuf F, Leu S, Zarka A, Khozin-Goldberg I, Khalilov I, Boussiba S. Cloning and molecular characterization of a novel acylCoA:diacylglycerol acyltransferase 1-like gene (PtDGAT1) from the diatom Phaeodactylum tricornutum. FEBS J. 2011;278:3651-66.

198. Cui Y, Zhao J, Wang Y, Qin S, Lu Y. Characterization and engineering of a dual-function diacylglycerol acyltransferase in the oleaginous marine diatom Phaeodactylum tricornutum. Biotechnol Biofuels. 2018;11:32.

199. Zhang Y, Pan Y, Ding W, Hu H, Liu J. Lipid production is more than doubled by manipulating a diacylglycerol acyltransferase in algae. GCB Bioenergy. 2021;13:185-200.

200. Hung C-H, Ho M-Y, Kanehara K, Nakamura Y. Functional study of diacylglycerol acyltransferase type 2 family in Chlamydomonas reinhardtii. FEBS Lett. 2013;587:2364-70.

201. Gong Y, Zhang J, Guo X, Wan X, Liang Z, Hu CJ, et al. Identification and characterization of PtDGAT2B, an acyltransferase of the DGAT2 
acyl-Coenzyme A: Diacylglycerol acyltransferase family in the diatom Phaeodactylum tricornutum. FEBS Lett. 2013;587:481-7.

202. Wu T, Mao X, Kou Y, Li Y, Sun H, He Y, et al. Characterization of microalgal acetyl-CoA synthetases with high catalytic efficiency reveals their regulatory mechanism and lipid engineering potential. J Agri Food Chem. 2019;67:9569-78.

203. Falarz LJ, Xu Y, Caldo KMP, Garroway CJ, Singer SD, Chen G. Characterization of the diversification of phospholipid:diacylglycerol acyltransferases in the green lineage. Plant J. 2020;103:2025-38.

204. Dahlqvist A, Stahl U, Lenman M, Banas A, Lee M, Sandager L, et al. Phospholipid : diacylglycerol acyltransferase: an enzyme that catalyzes the acyl-CoA-independent formation of triacylglycerol in yeast and plants. Proc Natl Acad Sci USA. 2000;97:6487-92.

205. Feng Y, Zhang Y, Ding W, Wu P, Cao X, Xue S. Expanding of phospholipid:diacylglycerol acyltransferase (PDAT) from Saccharomyces cerevisiae as multifunctional biocatalyst with broad acyl donor/ acceptor selectivity. Appl Biochem Biotechnol. 2019;188:824-35.

206. Eastmond PJ. SUGAR-DEPENDENT1 encodes a patatin domain triacylglycerol lipase that initiates storage oil breakdown in germinating Arabidopsis seeds. Plant Cell. 2006;18:665.

207. Barka F, Angstenberger M, Ahrendt T, Lorenzen W, Bode HB, Büchel C. Identification of a triacylglycerol lipase in the diatom Phaeodactylum tricornutum. Biochim Biophys Acta Mol Cell Biol Lipids. 2016;1861:239-48.

208. Siegler H, Valerius O, Ischebeck T, Popko J, Tourasse NJ, Vallon O, et al. Analysis of the lipid body proteome of the oleaginous alga Lobosphaera incisa. BMC Plant Biol. 2017:17:98.

209. Nobusawa T, Yamakawa-Ayukawa K, Saito F, Nomura S, Takami A, Ohta H. A homolog of Arabidopsis SDP1 lipase in Nannochloropsis is involved in degradation of de novo-synthesized triacylglycerols in the endoplasmic reticulum. Biochim Biophys Acta Mol Cell Biol Lipids. 2019;1864:1185-93.

210. Warakanont J, Li-Beisson Y, Benning C. LIP4 is involved in triacylglycerol degradation in Chlamydomonas reinhardtii. Plant Cell Physiol. 2019:60:1250-9.

211. El-Kouhen K, Blangy S, Ortiz E, Gardies A-M, Ferté N, Arondel V. Identification and characterization of a triacylglycerol lipase in Arabidopsis homologous to mammalian acid lipases. FEBS Lett. 2005;579:6067-73.

212. Goold H, Beisson F, Peltier G, Li-Beisson Y. Microalgal lipid droplets: composition, diversity, biogenesis and functions. Plant Cell Rep. 2015:34:545-55.

213. Huang AHC. Plant lipid droplets and their associated proteins: potential for rapid advances. Plant Physiol. 2018;176:1894-918.

214. IschebeckT, Krawczyk HE, Mullen RT, Dyer JM, Chapman KD. Lipid droplets in plants and algae: distribution, formation, turnover and function. Semin Cell Dev Biol. 2020;108:82-93.

215. Zienkiewicz K, Zienkiewicz A. Degradation of lipid droplets in plants and algae-right time, many paths, one goal. Front Plant Sci. 2020;11:1400

216. Nguyen HM, Baudet M, Cuiné S, Adriano J-M, Barthe D, Billon E, et al. Proteomic profiling of oil bodies isolated from the unicellular green microalga Chlamydomonas reinhardtii: with focus on proteins involved in lipid metabolism. Proteomics. 2011;11:4266-73.

217. Wang ZT, Ullrich N, Joo S, Waffenschmidt S, Goodenough U. Algal lipid bodies: stress induction, purification, and biochemical characterization in wild-type and starchless Chlamydomonas reinhardtii. Eukaryot Cell. 2009:8:1856-68.

218. Moellering ER, Benning C. RNA interference silencing of a major lipid droplet protein affects lipid droplet size in Chlamydomonas reinhardtii. Eukaryot Cell. 2010;9:97-106

219. Vieler A, Brubaker SB, Vick B, Benning C. A Lipid droplet protein of Nannochloropsis with functions partially analogous to plant oleosins. Plant Physiol. 2012;158:1562-9.

220. Nojima D, Yoshino T, Maeda Y, Tanaka M, Nemoto M, Tanaka T. Proteomics analysis of oil body-associated proteins in the oleaginous diatom. J Proteome Res. 2013;12:5293-301.

221. Davidi L, Levin Y, Ben-Dor S, Pick U. Proteome analysis of cytoplasmatic and plastidic $\beta$-carotene lipid droplets in Dunaliella bardawil. Plant Physiol. 2015;167:60-79.
222. Lupette J, Jaussaud A, Seddiki K, Morabito C, Brugière S, Schaller H, et al. The architecture of lipid droplets in the diatom Phaeodactylum tricornutum. Algal Res. 2019;38:101415.

223. You Z, Zhang Q, Peng Z, Miao X. Lipid droplets mediate salt stress tolerance in Parachlorella kessleri. Plant Physiol. 2019;181:510.

224. Tsai C-H, Zienkiewicz K, Amstutz CL, Brink BG, Warakanont J, Roston R, et al. Dynamics of protein and polar lipid recruitment during lipid droplet assembly in Chlamydomonas reinhardtii. Plant J. 2015;83:650-60.

225. Huang NL, Huang MD, Chen TLL, Huang AHC. Oleosin of subcellular lipid droplets evolved in green algae. Plant Physiol. 2013;161:1862-74.

226. Chen JCF, Tsai CCY, Tzen JTC. Cloning and secondary structure analysis of caleosin, a unique calcium-binding protein in oil bodies of plant seeds. Plant Cell Physiol. 1999:40:1079-86.

227. Blaby IK, Glaesener AG, Mettler T, Fitz-Gibbon ST, Gallaher SD, Liu B, et al. Systems-level analysis of nitrogen starvation-induced modifications of carbon metabolism in a Chlamydomonas reinhardtii starchless mutant. Plant Cell. 2013;25:4305-23.

228. Huang W, Ye J, Zhang J, Lin Y, He M, Huang J. Transcriptome analysis of Chlorella zofingiensis to identify genes and their expressions involved in astaxanthin and triacylglycerol biosynthesis. Algal Res. 2016;17:236-43.

229. Rodriguez-Concepcion M, Avalos J, Bonet ML, Boronat A, GomezGomez L, Hornero-Mendez D, et al. A global perspective on carotenoids: Metabolism, biotechnology, and benefits for nutrition and health. Prog Lipid Res. 2018;70:62-93.

230. Gwak Y, Hwang Y-S, Wang B, Kim M, Jeong J, Lee C-G, et al. Comparative analyses of lipidomes and transcriptomes reveal a concerted action of multiple defensive systems against photooxidative stress in Haematococcus pluvialis. J Exp Bot. 2014;65:4317-34.

231. Lohr M, Im C-S, Grossman AR. Genome-based examination of chlorophyll and carotenoid biosynthesis in Chlamydomonas reinhardtii. Plant Physiol. 2005:138:490-515.

232. Vranová E, Coman D, Gruissem W. Network analysis of the MVA and MEP pathways for isoprenoid synthesis. Annu Rev Plant Biol. 2013;64:665-700

233. Hagen C, Grunewald K. Fosmidomycin as an inhibitor of the nonmevalonate terpenoid pathway depresses synthesis of secondary carotenoids in flagellates of the green alga Haematococcus pluvialis. J Appl Bot. 2000;74:137-40.

234. Liang C, Zhang W, Zhang X, Fan X, Xu D, Ye N, et al. Isolation and expression analyses of methyl-d-erythritol 4-phosphate (MEP) pathway genes from Haematococcus pluvialis. J Appl Phycol. 2016;28:209-18.

235. Steiger S, Jackisch Y, Sandmann G. Carotenoid biosynthesis in Gloeobacter violaceus PCC4721 involves a single crtl-type phytoene desaturase instead of typical cyanobacterial enzymes. Arch Microbiol. 2005;184:207-14

236. Harada J, Nagashima KVP, Takaichi S, Misawa N, Matsuura K, Shimada K. Phytoene desaturase, $\mathrm{Crtl}$, of the purple photosynthetic bacterium, Rubrivivax gelatinosus, produces both neurosporene and lycopene. Plant Cell Physiol. 2001;42:1112-8.

237. Misawa N, Nakagawa M, Kobayashi K, Yamano S, Izawa Y, Nakamura K, et al. Elucidation of the Erwinia uredovora carotenoid biosynthetic pathway by functional analysis of gene products expressed in Escherichia coli. J Bacteriol. 1990:172:6704-12.

238. Fraser PD, Romer S, Shipton CA, Mills PB, Kiano JW, Misawa N, et al. Evaluation of transgenic tomato plants expressing an additional phytoene synthase in a fruit-specific manner. Proc Natl Acad Sci USA. 2002;99:1092-7.

239. Cordero B, Couso I, León R, Rodríguez H, Vargas M. Enhancement of carotenoids biosynthesis in Chlamydomonas reinhardtii by nuclear transformation using a phytoene synthase gene isolated from Chlorella zofingiensis. Appl Microbiol Biotechnol. 2011;91:341-51.

240. Ducreux LJM, Morris WL, Hedley PE, Shepherd T, Davies HV, Millam S, et al. Metabolic engineering of high carotenoid potato tubers containing enhanced levels of $\beta$-carotene and lutein. J Exp Bot. 2005;56:81-9.

241. Couso I, Vila M, Rodriguez H, Vargas MA, León R. Overexpression of an exogenous phytoene synthase gene in the unicellular alga Chlamydomonas reinhardtii leads to an increase in the content of carotenoids. Biotechnol Prog. 2011;27:54-60.

242. Chamovitz D, Sandmann G, Hirschberg J. Molecular and biochemicalcharacterization of herbicide-resistant mutants of cyanobacteria 
reveals that phytoene desaturation is a rate-limiting step in carotenoid biosynthesis. J Biol Chem. 1993;268:17348-53.

243. Steinbrenner J, Sandmann G. Transformation of the green alga Haematococcus pluvialis with a phytoene desaturase for accelerated astaxanthin biosynthesis. Appl Environ Microbiol. 2006;72:7477-84.

244. Li Y, Huang J, Sandmann G, Chen F. High-light and sodium chloride stress differentially regulate the biosynthesis of astaxanthin in Chlorella zofingiensis (Chlorophyceae). J Phycol. 2009;45:635-41.

245. Huang JC, Liu J, Li YT, Chen F. Isolation and characterization of the phytoene desaturase gene as a potential selective marker for genetic engineering of the astaxanthin-producing green alga Chlorella zofingiensis (Chlorophyta). J Phycol. 2008;44:684-90.

246. Grunewald K, Eckert M, Hirschberg J, Hagen C. Phytoene desaturase is localized exclusively in the chloroplast and up-regulated at the mRNA level during accumulation of secondary carotenoids in Haematococcus pluvialis (Volvocales, Chlorophyceae). Plant Physiol. 2000;122:1261-8.

247. Liu J, Gerken H, Huang J, Chen F. Engineering of an endogenous phytoene desaturase gene as a dominant selectable marker for Chlamydomonas reinhardtii transformation and enhanced biosynthesis of carotenoids. Process Biochem. 2013;48:788-95.

248. Taparia Y, Zarka A, Leu S, Zarivach R, Boussiba S, Khozin-Goldberg I. A novel endogenous selection marker for the diatom Phaeodactylum tricornutum based on a unique mutation in phytoene desaturase 1. Sci Rep. 2019;9:8217.

249. Liu J, Zhong Y, Sun Z, Huang J, Sandmann G, Chen F. One amino acid substitution in phytoene desaturase makes Chlorella zofingiensis resistant to norflurazon and enhances the biosynthesis of astaxanthin Planta. 2010;232:61-7

250. Cordero BF, Couso I, Leon R, Rodriguez H, Vargas MA. Isolation and characterization of a lycopene $\varepsilon$-cyclase gene of Chlorella (Chromochloris) zofingiensis. Regulation of the carotenogenic pathway by nitrogen and light. Mar Drugs. 2012;10:2069-2088.

251. Cordero BF, Obraztsova I, Martin L, Couso I, Leon R, Vargas MA, et al. Isolation and characterization of a lycopene beta-cyclase gene from the astaxanthin-producing green alga Chlorella zofingiensis (Chlorophyta). J Phycol. 2010;46:1229-38.

252. Couso I, Cordero BF, Vargas MÁ, Rodríguez H. Efficient heterologous transformation of Chlamydomonas reinhardtii npq2 mutant with the zeaxanthin epoxidase gene isolated and characterized from Chlorella zofingiensis. Mar Drugs. 2012;10:1955-76.

253. Huang J-C, Zhong Y-J, Liu J, Sandmann G, Chen F. Metabolic engineering of tomato for high-yield production of astaxanthin. Metab Eng. 2013;17:59-67.

254. Havaux M, Niyogi KK. The violaxanthin cycle protects plants from photooxidative damage by more than one mechanism. Proc Natl Acad Sci. 1999;96:8762-7.

255. Jahns P, Holzwarth AR. The role of the xanthophyll cycle and of lutein in photoprotection of photosystem II. Biochim Biophys Acta Bioenerg. 2012;1817:182-93.

256. Shah MMR, Liang Y, Cheng JJ, Daroch M. Astaxanthin-producing green microalga Haematococcus pluvialis: from single cell to high value commercial products. Front Plant Sci. 2016;7:531.

257. Fraser PD, Miura Y, Misawa N. In vitro characterization of astaxanthin biosynthetic enzymes. J Biol Chem. 1997;272:6128-35.

258. Huang JC, Chen F, Sandmann G. Stress-related differential expression of multiple beta-carotene ketolase genes in the unicellular green alga Haematococcus pluvialis. J Biotechnol. 2006;122:176-85.

259. Lotan T, Hirschberg J. Cloning and expression in Escherichia coli of the gene encoding $\beta$-C-4-oxygenase, that converts $\beta$-carotene to the ketocarotenoid canthaxanthin in Haematococcus pluvialis. FEBS Lett. 1995;364:125-8

260. Huang JC, Wang Y, Sandmann G, Chen F. Isolation and characterization of a carotenoid oxygenase gene from Chlorella zofingiensis (Chlorophyta). Appl Microbiol Biotechnol. 2006;71:473-9.

261. Huang $W$, Lin $Y$, He M, Gong Y, Huang J. Induced high-yield production of zeaxanthin, lutein, and $\beta$-carotene by a mutant of Chlorella zofingiensis. J Agri Food Chem. 2018;66:891-7.

262. Wang Y, Chen T. The biosynthetic pathway of carotenoids in the astaxanthin-producing green alga Chlorella zofingiensis. World J Microbiol Biotechnol. 2008;24:2927-32.
263. Zhong Y-J, Huang J-C, Liu J, Li Y, Jiang Y, Xu Z-F, et al. Functional characterization of various algal carotenoid ketolases reveals that ketolating zeaxanthin efficiently is essential for high production of astaxanthin in transgenic Arabidopsis. J Exp Bot. 2011;62:3659-69.

264. Perozeni F, Cazzaniga S, Baier T, Zanoni F, Zoccatelli G, Lauersen KJ, et al. Turning a green alga red: engineering astaxanthin biosynthesis by intragenic pseudogene revival in Chlamydomonas reinhardtii. Plant Biotechnol J. 2020;18:2053-67.

265. Peled E, Leu S, Zarka A, Weiss M, Pick U, Khozin-Goldberg I, et al. Isolation of a novel oil globule protein from the green alga Haematococcus pluvialis (Chlorophyceae). Lipids. 2011;46:851-61.

266. Goodson C, Roth R, Wang ZT, Goodenough U. Structural correlates of cytoplasmic and chloroplast lipid body synthesis in Chlamydomonas reinhardtii and stimulation of lipid body production with acetate boost. Eukaryot Cell. 2011;10:1592-606.

267. Miao F, Lu D, Li Y, Zeng M. Characterization of astaxanthin esters in Haematococcus pluvialis by liquid chromatography-atmospheric pressure chemical ionization mass spectrometry. Anal Biochem. 2006;352:176-81.

268. Shih MYS, Kane MA, Zhou P, Yen CLE, Streeper RS, Napoli JL, et al. Retinol esterification by DGAT1 is essential for retinoid homeostasis in murine skin. J Biol Chem. 2009;284:4292-9.

269. Yen CL, Monetti M, Burri BJ, Farese RV Jr. The triacylglycerol synthesis enzyme DGAT1 also catalyzes the synthesis of diacylglycerols, waxes, and retinyl esters. J Lipid Res. 2005;46:1502-11.

270. Zhu Q, Zeng D, Yu S, Cui C, Li J, Li H, et al. From golden rice to aSTARice: bioengineering astaxanthin biosynthesis in rice endosperm. Mol Plant. 2018;11:1440-8.

271. Hasunuma T, Miyazawa SI, Yoshimura S, Shinzaki Y, Tomizawa Kl, Shindo $\mathrm{K}$, et al. Biosynthesis of astaxanthin in tobacco leaves by transplastomic engineering. Plant J. 2008;55:857-68.

272. Ariizumi T, Kishimoto S, Kakami R, Maoka T, Hirakawa H, Suzuki Y, et al. Identification of the carotenoid modifying gene PALE YELLOW PETAL 1 as an essential factor in xanthophyll esterification and yellow flower pigmentation in tomato (Solanum lycopersicum). Plant J. 2014;79:453-65.

273. Watkins JL, Li M, McQuinn RP, Chan KX, McFarlane HE, Ermakova M, et al. A GDSL esterase/lipase catalyzes the esterification of lutein in bread wheat. Plant Cell. 2019;31:3092.

274. Fan L, Vonshak A, Zarka A, Boussiba S. Does astaxanthin protect Haematococcus against light damage? Z Naturforsch C. 1998;53:93-100.

275. Steinbrenner J, Linden $\mathrm{H}$. Regulation of two carotenoid biosynthesis genes coding for phytoene synthase and carotenoid hydroxylase during stress-induced astaxanthin formation in the green alga Haematococcus pluvialis. Plant Physiol. 2001;125:810-7.

276. Kobayashi M, Kakizono T, Nagai S. Enhanced carotenoid biosynthesis by oxidative stress in acetate-induced cyst cells of a green unicellular alga Haematococcus pluvialis. Appl Environ Microbiol. 1993;59:867-73.

277. Holtin K, Kuehnle M, Rehbein J, Schuler P, Nicholson G, Albert K. Determination of astaxanthin and astaxanthin esters in the microalgae Haematococcus pluvialis by LC-(APCI)MS and characterization of predominant carotenoid isomers by NMR spectroscopy. Anal Bioanal Chem. 2009;395:1613-22.

278. Schoefs B, Rmiki N-E, Rachadi J, Lemoine Y. Astaxanthin accumulation in Haematococcus requires a cytochrome $\mathrm{P} 450$ hydroxylase and an active synthesis of fatty acids. FEBS Lett. 2001;500:125-8.

279. Zhang Z, Sun D, Mao X, Liu J, Chen F. The crosstalk between astaxanthin, fatty acids and reactive oxygen species in heterotrophic Chlorella zofingiensis. Algal Res. 2016;19:178-83.

280. Yan J, Cheng R, Lin X, You S, Li K, Rong H, et al. Overexpression of acetylCOA synthetase increased the biomass and fatty acid proportion in microalga Schizochytrium. Appl Microbiol Biotechnol. 2013;97:1933-9.

281. Rengel R, Smith RT, Haslam RP, Sayanova O, Vila M, León R. Overexpression of acetyl-CoA synthetase (ACS) enhances the biosynthesis of neutral lipids and starch in the green microalga Chlamydomonas reinhardtii. Algal Res. 2018;31:183-93.

282. Avidan O, Pick U. Acetyl-CoA synthetase is activated as part of the PDH-bypass in the oleaginous green alga Chlorella desiccata. J Exp Bot. 2015;66:7287-98. 
283. Morales-Sánchez D, Kim Y, Terng EL, Peterson L, Cerutti H. A multidomain enzyme, with glycerol-3-phosphate dehydrogenase and phosphatase activities, is involved in a chloroplastic pathway for glycerol synthesis in Chlamydomonas reinhardtii. Plant J. 2017;90:1079-92.

284. Driver T, Trivedi DK, McIntosh OA, Dean AP, Goodacre R, Pittman JK. Two glycerol-3-phosphate dehydrogenases from Chlamydomonas have distinct roles in lipid metabolism. Plant Physiol. 2017;174:2083.

285. Wang X, Dong H-P, Wei W, Balamurugan S, Yang W-D, Liu J-S, et al. Dual expression of plastidial GPAT1 and LPAT1 regulates triacylglycerol production and the fatty acid profile in Phaeodactylum tricornutum. Biotechnol Biofuels. 2018;11:318.

286. Niu Y-F, Wang X, Hu D-X, Balamurugan S, Li D-W, Yang W-D, et al. Molecular characterization of a glycerol-3-phosphate acyltransferase reveals key features essential for triacylglycerol production in Phaeodacty/um tricornutum. Biotechnol Biofuels. 2016;9:60.

287. Iskandarov U, Sitnik S, Shtaida N, Didi-Cohen S, Leu S, Khozin-Goldberg I, et al. Cloning and characterization of a GPAT-like gene from the microalga Lobosphaera incisa (Trebouxiophyceae): overexpression in Chlamydomonas reinhardtii enhances TAG production. J Appl Phycol. 2016;28:907-19.

288. Balamurugan S, Wang X, Wang H-L, An C-J, Li H, Li D-W, et al. Occurrence of plastidial triacylglycerol synthesis and the potential regulatory role of AGPAT in the model diatom Phaeodacty/um tricornutum. Biotechnol Biofuels. 2017;10:97.

289. Ahmad I, Sharma AK, Daniell H, Kumar S. Altered lipid composition and enhanced lipid production in green microalga by introduction of brassica diacylglycerol acyltransferase 2. Plant Biotechnol J. 2015;13:540-50.

290. Niu YF, Zhang MH, Li DW, Yang WD, Liu JS, Bai WB, et al. Improvement of neutral lipid and polyunsaturated fatty acid biosynthesis by overexpressing a type 2 diacylglycerol acyltransferase in marine diatom Phaeodactylum tricornutum. Mar Drugs. 2013:11:4558-69.

291. Dinamarca J, Levitan O, Kumaraswamy GK, Lun DS, Falkowski PG. Overexpression of a diacylglycerol acyltransferase gene in Phaeodactylum tricornutum directs carbon towards lipid biosynthesis. J Phycol. 2017:53:405-14.

292. Manandhar-Shrestha K, Hildebrand M. Characterization and manipulation of a DGAT2 from the diatom Thalassiosira pseudonana: Improved TAG accumulation without detriment to growth, and implications for chloroplast TAG accumulation. Algal Res. 2015;12:239-48.

293. Chen C-Y, Kao A-L, Tsai Z-C, Chow T-J, Chang H-Y, Zhao X-Q, et al. Expression of type 2 diacylglycerol acyltransferse gene DGTT1 from Chlamydomonas reinhardtii enhances lipid production in Scenedesmus obliquus. Biotechnol J. 2016;11:336-44.
294. Klaitong P, Fa-aroonsawat S, Chungjatupornchai W. Accelerated triacylglycerol production and altered fatty acid composition in oleaginous microalga Neochloris oleoabundans by overexpression of diacylglycerol acyltransferase 2. Microb Cell Fact. 2017;16:61.

295. Trentacoste EM, Shrestha RP, Smith SR, Glé C, Hartmann AC, Hildebrand $M$, et al. Metabolic engineering of lipid catabolism increases microalgal lipid accumulation without compromising growth. Proc Natl Acad Sci USA. 2013;110:19748-53.

296. Li X, Pan Y, Hu H. Identification of the triacylglycerol lipase in the chloroplast envelope of the diatom Phaeodactylum tricornutum. Algal Res. 2018;33:440-7.

297. Rodriguez-Concepcion M. Supply of precursors for carotenoid biosynthesis in plants. Arch Biochem Biophys. 2010;504:118-22.

298. Ramos AA, Marques AR, Rodrigues M, Henriques N, Baumgartner A, Castilho $R$, et al. Molecular and functional characterization of a cDNA encoding 4-hydroxy-3-methylbut-2-enyl diphosphate reductase from Dunaliella salina. J Plant Physiol. 2009;166:968-77.

299. Paetzold H, Garms S, Bartram S, Wieczorek J, Uros-Gracia EM, RodriguezConcepcion M, et al. The isogene 1-deoxy-D-xylulose 5-phosphate synthase 2 controls isoprenoid profiles, precursor pathway allocation, and density of tomato trichomes. Mol Plant. 2010;3:904-16.

300. Jia B, Xie X, Wu M, Lin Z, Yin J, Lou S, et al. Understanding the functions of endogenous DOF transcript factor in Chlamydomonas reinhardtii. Biotechnol Biofuels. 2019;12:67.

301. Kang NK, Kim EK, Kim YU, Lee B, Jeong W-J, Jeong B-R, et al. Increased lipid production by heterologous expression of AtWRI1 transcription factor in Nannochloropsis salina. Biotechnol Biofuels. 2017;10:231.

302. Kwon S, Kang NK, Koh HG, Shin S-E, Lee B, Jeong B-R, et al. Enhancement of biomass and lipid productivity by overexpression of a bZIP transcription factor in Nannochloropsis salina. Biotechnol Bioeng. 2018:115:331-40.

303. Li D-W, Balamurugan S, Yang Y-F, Zheng J-W, Huang D, Zou L-G, et al. Transcriptional regulation of microalgae for concurrent lipid overproduction and secretion. Sci Adv. 2019;5:eaau3795.

304. Li Y, Huang J, Sandmann G, Chen F. Glucose sensing and the mitochondrial alternative pathway are involved in the regulation of astaxanthin biosynthesis in the dark-grown Chlorella zofingiensis (Chlorophyceae). Planta. 2008:228:735-43.

\section{Publisher's Note}

Springer Nature remains neutral with regard to jurisdictional claims in published maps and institutional affiliations.
Ready to submit your research? Choose BMC and benefit from:

- fast, convenient online submission

- thorough peer review by experienced researchers in your field

- rapid publication on acceptance

- support for research data, including large and complex data types

- gold Open Access which fosters wider collaboration and increased citations

- maximum visibility for your research: over $100 \mathrm{M}$ website views per year

At BMC, research is always in progress.

Learn more biomedcentral.com/submissions 